Sharif University of Technology
Scientia Iranica
Transactions E: Industrial Engineering
hCIttp://scientiairanica.sharif.edu
IRAN I CA

Research Note

\title{
On auxiliary information based improved EWMA median control charts
}

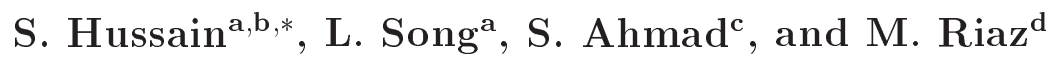 \\ a. School of Mathematical Sciences, Dalian University of Technology, Dalian, 116024, P.R. China. \\ b. Department of Mathematics, COMSATS Institute of Information Technology, Attock, 43600, Pakistan. \\ c. Department of Mathematics, COMSATS Institute of Information Technology, Wah Cantt, 47040 , Pakistan. \\ d. Department of Mathematics and Statistics, King Fahad University of Petroleum and Minerals, Dhahran, 31261, Saudi Arabia.
}

Received 23 January 2016; received in revised form 4 August 2016; accepted 19 December 2016

\author{
KEYWORDS \\ Average Run Length \\ (ARL); \\ Auxiliary information; \\ EWMA control \\ charts; \\ Extra Quadratic Loss \\ (EQL); \\ Median control charts; \\ Performance \\ Comparison Index \\ (PCI); \\ Relative Average Run \\ Length (RARL).
}

\begin{abstract}
Process monitoring is a continuous process for improving the quality. Control chart is a process monitoring tool of SPC tool kit that plays an important role in providing widespread monitoring, to observe the changes in parameters. Mostly, the mean control charts are used for monitoring of process location. In a perfect situation, when there are no outliers, the mean charts are more efficient than median control charts. In reality, data is not always free from outliers, so the median charts are considered as the best for monitoring location parameters. The use of an auxiliary variable in a control chart may be the cause of efficiency gain. The current article considers EWMA median charts based on auxiliary variable(s). Different run length performance measures are considered to expedite the proposed charts in both contaminated and uncontaminated process environments under multivariate normal distributions. An illustrative example is provided to validate the performance of the proposed charts. From the results, we deduce that the performance of median control charts is much better than that of mean control charts in the presence of outliers; moreover, the performance of control charts can be enhanced by using more auxiliary variables.
\end{abstract}

(C) 2018 Sharif University of Technology. All rights reserved.

\section{Introduction}

Statistical Process Control (SPC) is a process monitoring tool kit containing different methods that are used to examine the process [1]. The control charts are significantly and effectively used to monitor the variation in the manufacturing or service process mechanisms [2]. A variety of control charts have been studied in literature taking into consideration the Shewhart,

*. Corresponding author.

E-mail addresses: shahid_libra@hotmail.com \& shahidhussain@ciit-attock.edu.pkx (S.Hussain);

lxsongdl@163.com (L. Song); shabbirahmad786@yahoo.com (S. Ahmad); riaz76qau@yahoo.com (M. Riaz)

doi: $10.24200 /$ sci.2017.4432 cumulative sum (CUSUM), and the Exponentially Weighted Moving Average (EWMA) control charts. The effective performance of these processes largely depends on the specific size of the shifts with regards to the variables under consideration. In the presence of shifts in process parameters, quick detection always benefits the manufacturing process and thus improves the quality of the process. There are two types of causes involved in any process; assignable causes and common causes. The random and innate part of a certain process has non-obvious reasons known as common causes and assignable or transactional causes are non-random. The assignable causes have some premeditated factors of concerned reasons having the key effect on the stability of the process and if these causes exist, then the process is called an out-of-control 
process. If we manage the variations in the output of a process by removing these assignable causes, then the results in the process will be improved. In the presence of shifts in process parameters, quick detection is always beneficial to improve the quality of the process. These shifts may exist in process mean or in process dispersion, which are commonly used parameters.

Quality control charts have also found implementation in the fields of production, statistics, and finance. They entail two main types, namely, memory less control charts and memory control charts, in calculating the efficiency of products and in process monitoring mechanism. The Shewhart control charts are also known as memory less control charts and have been commonly and extensively used for observing the variation in the process of production. These charts are less efficient in showing performance for moderate and small shifts in process' parameters, which is the main disadvantage of Shewhart control charts [2]. Because we rarely determine the exact shift value of a given process before it is relatively detected, it is important to look at a wide range of known and unknown mean shifts. In order to overcome these drawbacks associated with these charts, an alternative approach is considered taking into account the cumulative sum (CUSUM) and Exponentially Weighted Moving Average (EWMA) control charts, which are memory control charts. The CUSUM and EWMA control charts were initially studied by Page [3] and Roberts [4], respectively. Memory control charts utilize current information as well as past information for detecting moderate and small shifts in process parameters while, on the other hand, memory less control charts only use current information and ignore past information. As the EWMA control charts are sensitive and efficient relative to Shewhart charts; thus, they are very popular in detecting small shifts in the process.

The design strategies and properties of EWMA control charts for monitoring of process mean have been meticulously explored in many papers (see, e.g. [5-11]). Many researchers studied EWMA control charts for variance, e.g. [12-23].

Statistics such as sample median or the range are used in the statistical process control applications. However, less attention has been paid for utilizing these statistics as an instrumental tool for monitoring process parameters in the literature of quality control. Some researchers suggested median based control charts as well as quantile-based control charts. For example, median rankit control chart was suggested by Kanji and Arif [24,25] and Chen and Chiou [26]; Yang et al. [27] proposed CUSUM-median control charts; Generally Weighted Moving Average (GWMA)-median control charts were suggested by Sheu and Yang [28,29]; and mixed EWMA median and range control charts were suggested by Castagliola [30]. Recently Castagliola et al. [31] proposed EWMA median charts with estimated parameters.

The concept of using auxiliary information is based on the application of estimation and survey sampling techniques. For example, during the study of any chemical production process, the temperature may assist an auxiliary variable, which can be easily observed. After identifying the auxiliary variable(s) with study variable, the precision of an estimator can be enhanced by using earlier information on an auxiliary variable (cf. [32]). A number of studies in the existing literature suggest that variables' auxiliary characteristic information can be used to attain higher efficiency, one may refer to [33-42]. This idea of auxiliary information is also used in various control charting procedures to enhance the monitoring of quality characteristic, such as cause-selecting control charts, auxiliary information-based control charts, regressionadjusted control charts, and ranked set-based control charts. For more details, see [20,33-35,37,43-52].

Khoo [53] presented the control chart structure of sample median based on the process which is normally distributed. Ahmad et al. [37] added some more median estimators under normal distribution based on the estimators using one and two auxiliary variables under Shewhart set up. The EWMA control charts outperform the Shewhart control charts to identify the moderate and small shifts. By taking inspiration from these works, we propose a set of EWMA type median control charts. For these charting structures, we consider the properties of estimators under study using simple random sampling from univariate, bivariate, and trivariate normal distributions.

In our study, we have considered the assumptions of normality (as many researchers used in literature, e.g., $[33,34,37,54-56])$ to evaluate the performance of proposed EWMA charts. We have also studied the run length properties for the purpose of performance evaluation of understudy EWMA charts. The rest of the paper is organized as follows: Section 2 briefly explains the set of median estimators and EWMA charting structures for these estimators; discussion about the performance measures for evaluating control charts is presented in Section 3. Section 4 is based on the description of simulation study comparison among various median type control charts. Section 5 exhibits an illustrative example. The outcomes of this study are concluded in Section 6 .

\section{Median estimators and improved EWMA charts}

Let $Y$ denote the quality characteristic and auxiliary characteristics are denoted by $X$ and $Z$. The means and standard deviations of $X, Y$, and $Z$ are represented by $\mu_{x}, \mu_{y}, \mu_{z}$, and $\sigma_{x}, \sigma_{y}$, and $\sigma_{z}$, respectively. $M_{x}$, 
$M_{y}$, and $M_{z}$ represent the population medians of $X, Y$, and $Z$ while the sample medians are shown by $\hat{M}_{x}, \hat{M}_{y}$, and $\hat{M}_{z}$. The correlations between $Y \& X, Z \& Y$, and $X \& Z$ are denoted by $\rho_{x y}, \rho_{y z}$, and $\rho_{z x}$. Moreover, we assume that the coefficients of variations for $Z$ and $X$ are represented by $C_{z}$ and $C_{x}$ while the coefficients of kurtosis for $X$ and $Z$ are shown by $\beta_{2}(x)$ and $\beta_{2}(z)$, respectively. Following the above preliminaries, we have considered a set of mean and median estimators based on auxiliary characteristics, which are defined below:

\section{Usual median estimator}

$$
M_{1}=\hat{M}_{y} \text {. }
$$

The mean and standard deviation of $M_{1}$ are, respectively, defined as $\mu_{M_{1}}=M_{y}$, and: $\sigma_{M_{1}}=0.5$ $\sqrt{\psi f_{y}^{2}\left(M_{y}\right)}$, where $\psi=(N-n) / N n$.

Kuk and Mak [57] suggested a ratio estimator for population median as:

$$
M_{2}=\hat{M}_{y} \frac{M_{x}}{\hat{M}_{x}} .
$$

Up to the first order approximation, the mean and standard deviation of $M_{2}$ are shown in Box I, where $f_{x}\left(M_{x}\right), f_{y}\left(M_{y}\right)$, and $f_{z}\left(M_{z}\right)$, are the ordinates of distributions of $X, Y$, and $Z$ at $M_{x}, M_{y}$, and $M_{z}$ respectively. Also, $\phi_{x y}=\phi_{\left(\hat{M}_{x}, \hat{M}_{y}\right)}=4 P_{11}(x, y)-$ 1 , where $P_{11}(x, y)$ is the proportion of units in the population with $X \leq M_{x}$ and $Y \leq M_{y}$.

Ahmad et al. [37] suggested a median estimator, which was a modified form of mean estimator $\left(\bar{y}_{\mathrm{SD}}\right)$ suggested by Sisodia and Dwived [58]:

$$
M_{3}=\hat{M}_{y}\left(\frac{M_{x}+C_{x}}{\hat{M}_{x}+C_{x}}\right) \text {. }
$$

They also [37] suggested the median form of mean estimator $\left(\bar{y}_{\mathrm{SK}}\right)$ suggested by Singh and Kakran [59]:

$$
M_{4}=\hat{M}_{y}\left(\frac{M_{x}+\beta_{2}(x)}{\hat{M}_{x}+\beta_{2}(x)}\right) .
$$

Upadhyaya and Singh [60] suggested two new ratio type estimators for mean $\left(\bar{y}_{\mathrm{US}_{1}}\right.$ and $\left.\bar{y}_{\mathrm{US}_{2}}\right)$ and Ahmad et al. [37] proposed their median estimators:

$$
\begin{aligned}
& M_{5}=\hat{M}_{y}\left(\frac{M_{x} \beta_{2}(x)+C_{x}}{\hat{M}_{x} \beta_{2}(x)+C_{x}}\right), \\
& M_{6}=\hat{M}_{y}\left(\frac{M_{x} C_{x}+\beta_{x}(x)}{\hat{M}_{x} C_{x}+\beta_{x}(x)}\right) .
\end{aligned}
$$

Up to the first order approximation, the mean and standard deviation of $M_{3}, M_{4}, M_{5}$, and $M_{6}$ are shown in Box II, where:

$$
\begin{aligned}
\Delta_{1 x} & =\frac{M_{x}}{M_{x}+C_{x}}, & \Delta_{2 x} & =\frac{M_{x}}{M_{x}+\beta_{2}(x)}, \\
\Delta_{3 x} & =\frac{M_{x} \beta_{2}(x)}{M_{x} \beta_{2}(x)+C_{x}}, & \Delta_{4 x} & =\frac{M_{x} C_{x}}{M_{x} C_{x}+\beta_{2}(x)}, \\
\Delta_{1 z} & =\frac{M_{z}}{M_{z}+C_{z}}, & \Delta_{2 z} & =\frac{M_{z}}{M_{z}+\beta_{2}(z)},
\end{aligned}
$$

$$
\mu_{M_{2}}=\frac{0.25 M_{y}}{M_{x}^{2} f_{x}^{2}\left(M_{x}\right)}\left[\psi\left\{1-\phi_{x y} \frac{M_{x} f_{x}\left(M_{x}\right)}{M_{y} f_{y}\left(M_{y}\right)}\right\}+4 M_{x}^{2} f_{x}^{2}\left(M_{x}\right)\right],
$$

and:

$$
\sigma_{M_{2}}=\sqrt{\frac{0.25 \psi}{f_{y}^{2}\left(M_{y}\right)}\left[1+\left(\frac{M_{y} f_{y}\left(M_{y}\right)}{M_{x} f_{x}\left(M_{x}\right)}\right)^{2}\left\{1-2 \phi_{x y} \frac{M_{x} f_{x}\left(M_{x}\right)}{M_{y} f_{y}\left(M_{y}\right)}\right\}\right]} .
$$

$$
\begin{aligned}
\mu_{M_{i}} & =\frac{0.25 M_{y}}{M_{x}^{2} f_{x}^{2}\left(M_{x}\right)}\left[\psi \Delta_{j x}\left\{\Delta_{j x}-\phi_{x y} \frac{M_{x} f_{x}\left(M_{x}\right)}{M_{y} f_{y}\left(M_{y}\right)}\right\}+4 M_{x}^{2} f_{x}^{2}\left(M_{x}\right)\right], \\
\sigma_{M_{i}} & =\sqrt{\frac{0.25 \psi}{f_{y}^{2}\left(M_{y}\right)}\left[1+\Delta_{j x}\left(\frac{M_{y} f_{y}\left(M_{y}\right)}{M_{x} f_{x}\left(M_{x}\right)}\right)^{2}\left\{\Delta_{j x}-2 \phi_{x y} \frac{M_{x} f_{x}\left(M_{x}\right)}{M_{y} f_{y}\left(M_{y}\right)}\right\}\right]},
\end{aligned}
$$

for $i(j)=3,4,5$ and $6(1,2,3$ and 4$)$, respectively. 


$$
\Delta_{3 z}=\frac{M_{z} \beta_{2}(z)}{M_{z} \beta_{2}(z)+C_{z}}, \quad \Delta_{4 z}=\frac{M_{z} C_{z}}{M_{z} C_{z}+\beta_{2}(z)} .
$$

Ahmad et al. [37] introduced the estimator of median involving two auxiliary variables:

$$
M_{7}=\hat{M}_{y}\left(\frac{M_{x}}{\hat{M}_{x}}\right)^{\theta_{1}}\left(\frac{M_{z}}{\hat{M}_{z}}\right)^{\theta_{2}}
$$

where:

$$
\begin{aligned}
& \theta_{1}=\frac{\left(\phi_{x y}-\phi_{y z} \phi_{x z}\right) M_{x} f_{x}+\left(M_{x}\right)}{\left(1-\phi_{x z}^{2}\right) M_{y} f_{y}\left(M_{y}\right)}, \\
& \theta_{2}=\frac{\left(\phi_{y z}-\phi_{x y} \phi_{x z}\right) M_{z} f_{z}\left(M_{z}\right)}{\left(1-\phi_{x z}^{2}\right) M_{y} f_{y}\left(M_{y}\right)} .
\end{aligned}
$$

Up to the first order approximation, the mean and standard deviation of $M_{7}$ are, respectively:

$$
\begin{aligned}
\mu_{M_{7}}= & \frac{0.125\left(1-\phi_{x z}^{2}\right)^{-1}}{M_{y} f_{y}^{2}\left(M_{y}\right)}\left[\psi \frac{M_{y} f_{y}\left(M_{y}\right)}{M_{x} f_{x}\left(M_{x}\right)}\left(\phi_{x y}-\phi_{y z} \phi_{x z}\right)\right. \\
& +\psi \frac{M_{y} f_{y}\left(M_{y}\right)}{M_{z} f_{z}\left(M_{z}\right)}\left(\phi_{y z}-\phi_{x y} \phi_{x z}\right) \\
& -\psi\left(\phi_{x y}^{2}+\phi_{y z}^{2}-2 \phi_{x y} \phi_{y z} \phi_{x z}\right) \\
& \left.+8 M_{y}^{2} f_{y}^{2}\left(M_{y}\right)\left(1-\phi_{x z}^{2}\right)\right]
\end{aligned}
$$

and:

$$
\sigma_{M_{7}}=\sqrt{\frac{0.25 \psi}{f_{y}^{2}\left(M_{y}\right)}\left[1-\left(\frac{\phi_{x y}^{2}+\phi_{y z}^{2}-2 \phi_{x y} \phi_{y z} \phi_{x z}}{1-\phi_{x z}^{2}}\right)\right]} .
$$

Gupta and Shabbir [61] proposed generalized ratio type estimators of $\left(\bar{y}_{\mathrm{SD}}, \bar{y}_{\mathrm{SK}}, \bar{y}_{\mathrm{US}_{1}}, \bar{y}_{\mathrm{US}_{2}}\right)$ using two auxiliary variables. Ahmad et al. [37] introduced their median estimators:

$$
\begin{aligned}
& M_{8}=\hat{M}_{y}\left(\frac{M_{x}+C_{x}}{\hat{M}_{x}+C_{x}}\right)^{\gamma_{1}}\left(\frac{M_{z}+C_{z}}{\hat{M}_{z}+C_{z}}\right)^{\gamma_{2}}, \\
& M_{9}=\hat{M}_{y}\left(\frac{M_{x}+\beta_{2}(x)}{\hat{M}_{x}+\beta_{2}(x)}\right)^{\gamma_{1}}\left(\frac{M_{z}+\beta_{2}(z)}{\hat{M}_{z}+\beta_{2}(z)}\right)^{\gamma_{2}}, \\
& M_{10}=\hat{M}_{y}\left(\frac{M_{x} \beta_{2}(x)+C_{x}}{\hat{M}_{x} \beta_{2}(x)+C_{x}}\right)^{\gamma_{1}}\left(\frac{M_{z} \beta_{2}(z)+C_{z}}{\hat{M}_{z} \beta_{2}(z)+C_{z}}\right)^{\gamma_{2}},
\end{aligned}
$$

and:

$$
M_{11}=\hat{M}_{y}\left(\frac{M_{x} C_{x}+\beta_{2}(x)}{\hat{M}_{x} C_{x}+\beta_{2}(x)}\right)^{\gamma_{1}}\left(\frac{M_{z} C_{z}+\beta_{2}(z)}{\hat{M}_{z} C_{z}+\beta_{2}(z)}\right)^{\gamma_{2}} .
$$

Up to first order approximation, the means and standard deviations of $M_{8}, M_{9}, M_{10}$, and $M_{11}$ are:

$$
\begin{aligned}
\mu_{M_{i}}= & \frac{0.125\left(1-\phi_{x z}^{2}\right)^{-1}}{M_{y} f_{y}^{2}\left(M_{y}\right)} \\
& {\left[\psi \Delta_{j x} \frac{M_{y} f_{y}\left(M_{y}\right)}{M_{x} f_{x}\left(M_{x}\right)}\left(\phi_{x y}-\phi_{y z} \phi_{x z}\right)\right.} \\
& +\psi \Delta_{j z} \frac{M_{y} f_{y}\left(M_{y}\right)}{M_{z} f_{z}\left(M_{z}\right)}\left(\phi_{y z}-\phi_{x y} \phi_{x z}\right) \\
& -\psi\left(\phi_{x y}^{2}+\phi_{y z}^{2}-2 \phi_{x y} \phi_{y z} \phi_{x z}\right) \\
& \left.+8 M_{y}^{2} f_{y}^{2}\left(M_{y}\right)\left(1-\phi_{x z}^{2}\right)\right]
\end{aligned}
$$

and:

$$
\sigma_{M_{i}}=\sqrt{\frac{0.25 \psi}{f_{y}^{2}\left(M_{y}\right)}\left[1-\left(\frac{\phi_{x y}^{2}+\phi_{y z}^{2}-2 \phi_{x y} \phi_{y z} \phi_{x z}}{1-\phi_{x z}^{2}}\right)\right]},
$$

where $i(j)=8,9,10$ and $11(1,2,3$ and 4$)$, respectively;

$$
\gamma_{1}=\frac{\left(\phi_{x y}-\phi_{y z} \phi_{x z}\right) M_{x} f_{x}\left(M_{x}\right)}{\Delta_{j x}\left(1-\phi_{x z}^{2}\right) M_{y} f_{y}\left(M_{y}\right)},
$$

and:

$$
\gamma_{2}=\frac{\left(\phi_{y z}-\phi_{x y} \phi_{x z}\right) M_{z} f_{z}\left(M_{z}\right)}{\Delta_{j x}\left(1-\phi_{x z}^{2}\right) M_{y} f_{y}\left(M_{y}\right)} .
$$

The properties of these estimators can be found in $[37,61,62]$. In this article, we use the EWMA control charting structure for median estimators $M_{1}-M_{11}$ as discussed by Ahmad et al. [37] in Shewhart set up. The description of EWMA design for these estimators is as follows.

Roberts [4] first introduced the EWMA control charts and claimed that for moderate and small shifts in the process, the performance of EWMA type control charts was superior to that of the Shewhart-type control charts. The EWMA's statistic is a comprehensive mixture of the past and current information and is defined as:

$$
Z_{j}=\lambda M_{j}+(1-\lambda) Z_{j-1},
$$

where $\lambda$ is the smoothing parameter that ranges from 0 to $1(0<\lambda \leq 1), Z_{j-1}$ is the past information, and $M_{j}$ is the current information (for $j=1,2,3, \cdots$ ). A substituted form of the statistic given in Eq. (1) can be expressed as:

$$
Z_{j}=\sum_{i=1}^{j-1} \lambda(1-\lambda)^{i} M_{j-1}+(1-\lambda)^{j} Z_{0} .
$$

When sample observations become less recent, the weights $\lambda(1-\lambda)^{i}$ in Eq. (2) decrease geometrically. Since the sensitivity and efficiency of these charts 
amplify using a smaller value of $\lambda, \lambda$ can be called the sensitivity parameter of EWMA charts. EWMA control charts and Shewhart control charts will be the same if $\lambda=1$. This implies that all weights were given in the recent information, not in the past information. It is, therefore, precise to conclude that Shewharttype control charts are the special case of EWMA control charts. The estimated value of the average of preliminary data can be used if we have no detailed information about target mean. EWMA statistic has the following mean and variance:

$$
\begin{aligned}
& \operatorname{Mean}\left(Z_{j}\right)=\mu_{0}, \\
& \operatorname{Variance}\left(Z_{j}\right)=\sigma^{2}\left\{\frac{\lambda}{2-\lambda}\left(1-(1-\lambda)^{2 j}\right)\right\},
\end{aligned}
$$

where $\sigma^{2}$ is the process variance. Here, we use either the known value of $\sigma^{2}$, i.e. $\sigma_{0}^{2}$, or estimated value from samples of the initial in-control process. We carry out an analysis in which the known parameters are considered. Given the above results, the EWMA control charts' structure for median estimators is given as:

$$
\begin{aligned}
& \mathrm{LCL}=\mu_{0}-L \sigma \sqrt{\frac{\lambda}{2-\lambda}\left(1-(1-\lambda)^{2 j}\right),} \\
& \mathrm{CL}=\mu_{0}, \\
& \mathrm{UCL}=\mu_{0}+L \sigma \sqrt{\frac{\lambda}{2-\lambda}\left(1-(1-\lambda)^{2 j}\right)} .
\end{aligned}
$$

The range of these control limits is determined by $L$, and its values depend on the choice of the constant $\lambda$ and predefined value of $\mathrm{ARL}_{0}$. These limits are also known as time varying limits of EWMA type control charts. With the large value of $j$, the limits given in Eq. (4) converge to the constant limits, which can be shown as:

$$
\begin{aligned}
& \mathrm{LCL}=\mu_{0}-L \sigma \sqrt{\frac{\lambda}{2-\lambda}}, \\
& \mathrm{CL}=\mu_{0}, \\
& \mathrm{UCL}=\mu_{0}+L \sigma \sqrt{\frac{\lambda}{2-\lambda}} .
\end{aligned}
$$

It is worth mentioning that when the number of samples increases, the factor $\left(1-(1-\lambda)^{2 j}\right)$ in Eq. (4) tends to 1 .

\section{Performance measures and simulation procedure}

The performance of control charts can be measured by using different techniques. Usually, there are two classifications of these measures: measures for specific shifts and measures based on overall shifts in the process parameters. We evaluate the performances of the EWMA median and mean control charts using ARL, EQL, RARL, and PCI in an uncontaminated environment. The ARL can evaluate control chart's performance at any specific shift point while, over the whole range of shifts, the effectiveness of control charts can be evaluated by EQL, RARL, and PCI. The descriptions of these measures are given below:

- Average Run Length (ARL): Mainly two values of ARL, which are denoted by $\mathrm{ARL}_{0}$ and $\mathrm{ARL}_{1}$, are used to evaluate the performance. Under the conditions of the in-control situation, the expected number of samples before an out-of-control false alarm is detected and defined as $\mathrm{ARL}_{0}$, while the expected number of samples when the process is shifted to an out-of-control situation is defined as $\mathrm{ARL}_{1}$. Wu et al. [63] stated that charts were said to be more proficient provided that they had a smaller $\mathrm{ARL}_{1}$ at more points. In general, larger values of $\mathrm{ARL}_{0}$ and smaller values of $\mathrm{ARL}_{1}$ are desirable;

- Extra Quadratic Loss (EQL): It can be described as a weighted average run length over the whole process domain of shifts $\left(\delta_{\min }<\delta<\delta_{\max }\right)$ using a square of shift $\left(\delta^{2}\right)$ as weights. Mathematically, this definition follows:

$$
\mathrm{EQL}=\frac{1}{\delta_{\max }-\delta_{\min }} \int_{\delta_{\min }}^{\delta_{\max }} \delta^{2} \operatorname{ARL}(\delta) d \delta,
$$

where $\operatorname{ARL}(\delta)$ indicates the ARL value of a specific chart at the shift $\delta$;

- Relative Average Run Length (RARL): It evaluates the average of the quotients between ARL of a specific chart, i.e. $\operatorname{ARL}(\delta)$, and ARL of a standard chart, i.e. $\operatorname{ARL}_{\text {standard }}(\delta)$. Mathematically, RARL is defined as:

$$
\operatorname{RARL}=\frac{1}{\delta_{\max }-\delta_{\min }} \int_{\delta_{\min }}^{\delta_{\max }} \frac{\operatorname{ARL}(\delta)}{\operatorname{ARL}_{\text {standard }}(\delta)} d \delta .
$$

A standard chart is selected based on the lowest value of EQL and the value of its RARL will be one. However, this value will be greater than one for other charts. The difference between RARLs of other charts and the standard chart shows the amount of inferior performance of a chart to that of the standard chart. We use numerical integration method for the calculations of RARL and EQL for all EWMA charts based on median estimators $M_{1}-M_{11}$ and mean estimators $T_{1}-T_{11}$;

- Performance Comparison Index (PCI): It is the quotient of dividing the EQL of a chart to the EQL of the superior chart, that is:

$$
\mathrm{PCI}=\frac{\mathrm{EQL}}{\mathrm{EQL}_{\text {superior }}} \text {. }
$$


Therefore, the value of PCI will be one for the superior chart and more than one for other competing charts. The same definitions are also provided by Montgomery [2], Abbasi et al. [13], and Ahmad et al. [37].

\subsection{Simulation procedure}

Different approaches can be used for calculating the ARL, for example, Monte Carlo simulations, integral equations, and Markov chains approximations. To obtain the ARL values, we choose the Monte Carlo simulation approach. The simulation algorithms of all median estimators for the calculations of ARL values are developed using $\mathrm{R}$ language software package. In our study, we use 100,000 Monte Carlo simulations to get the results of estimators taking into account 5,000 repetitions. For the number of Monte Carlo simulations in quality control, one may refer to Schaffer and Kim [64].

An appropriate number of samples, say 100,000, having a fixed size, $n$, are produced by $N\left(\mu+\delta \sigma, \sigma^{2}\right)$, where $\mu$ and $\sigma^{2}$ are mean and variance of the process, respectively. $\delta$ is a quantity of shift in the process. Generally, $\delta=0$ shows the in-control situation, while $\delta \neq 0$ represents the out-of-control situation. For $\delta=0$, the $\mathrm{ARL}_{0}$ values are calculated with the help of their corresponding $L \mathrm{~s}$ and for $\delta \neq 0, \mathrm{ARL}_{1}$ values are calculated by presenting various shifts in the process.

\section{Comparative analysis}

For the purpose of analyzing run length characteristics of our charts, random samples are generated from trivariate normal distribution of size $n$, using different correlation combinations of high, moderate, and weak correlations, $\rho_{x y}, \rho_{y z}$, and $\rho_{z x}$. We determine the EWMA control structures defined in Eq. (5) for both median $\left(M_{1}-M_{11}\right)$ and mean $\left(T_{1}-T_{11}\right)$ control charts using $\mathrm{ARL}_{0}=200 ; n=5,10,15$, and $\lambda=$ $1.0,0.75,0.5,0.25$. We may get Shewhart structure as a special case of EWMA control charts when $\lambda=1.0$. We calculate the values of $L$ for each chart that fix the control limits Eq. (5) to obtain incontrol ARL $\left(\mathrm{ARL}_{0}=200\right)$ and the out-of-control $\operatorname{ARL}\left(\mathrm{ARL}_{1}\right)$ values at discrete points of shift as $\delta=$ $0.10,0.15,0.20,0.25,0.50,0.75, \cdots, 3.0$.

\subsection{Comparison of control charts under uncontaminated environment}

Without loss of generality, the values of 5 and 1 are respectively, used for $\mu_{i} \mathrm{~s}$ and $\sigma_{i} \mathrm{~s}$. After fixing $\mathrm{ARL}_{0}$ at 200 and with multiple choices of $\lambda$, the ARL analysis is performed for estimators $M_{1}-M_{11}$ and $T_{1}-T_{11}$ based on the results of means and standard deviations of these estimators. Under the uncontaminated environment, we have compared mean and median control charts in three different ways: i. Comparison of median control charts: To compare the performances of EWMA control charts using different median estimators, we construct ARL-curves for control charts based on $M_{i}(i=1,2, \cdots, 11)$; the ARL values are used at varying shifts. These ARL curves are presented in Figure 1 and are based on some selected combinations of correlations. For these curves, we used $\log$ (ARL) on $Y$-axis for improved scaling. Figure 1 represents the comparison of all median control charts.

By the comparison of all median control charts, it can be seen that for all categories (high, moderate, and low) of correlation combinations $\rho_{x y}, \rho_{y z}$, and $\rho_{z x}$, as considered in Figure 1, the ARL curves indicate that the performance of estimators $M_{8}-M_{11}$ is unparalleled among all estimators included in this study. The superiority order of remaining estimators $M_{1}-M_{7}$ is observed as: The ARL output of $M_{7}$ based control chart is the best for the same values of $\rho_{x y}$ and $\rho_{z x}$; for high values of $\rho_{x y}$ and $\rho_{z x}$, the superiority order of control charts is observed as: $M_{4}, M_{3}, M_{5}$, and $M_{2}$ followed by $M_{6}$ and $M_{1}$; for moderate values of $\rho_{x y}$ and high or moderate values of $\rho_{y z}$, the superiority order of charting structures will be given by $M_{6}$, $M_{4}, M_{1}$, and $M_{3}$ followed by $M_{5}$ and $M_{2}$. The performance of $M_{1}$ is the most inferior, in general, for the high values of $\rho_{x y}$ and most inferior performance is demonstrated by $M_{2}$ if $\rho_{x y}$ is moderate.

EWMA control charts for median estimators $M_{1}-M_{11}$ are compared by some suitable properties of run length, e.g. RARL, EQL, and PCI. Table 1 presents the values of EQL, RARL, and PCI for all correlation combinations at $\lambda=0.25$ and sample size 5. In Tables 2 and 3, we present the values of EQL, RARL, and PCI with all four values of $\lambda$ and selective correlation combinations of $\rho_{x y}, \rho_{y z}$, and $\rho_{z x}$ for the sample sizes of 10 and 15 , respectively.

It can be observed from EQL values provided in Tables 1-3 that the efficiency of the charts improved with the increment in sample size. EWMA median charts are more efficient at the lower values of $\lambda$ and small shifts in the process, which can also be observed in our study. For the EQL values, the EWMA median charts become more efficient at the lowest value of $\lambda$ for the detection of process shift in the mean. It is also observed from EQL values that EWMA median charting structures (at $\lambda=0.75,0.50,0.25$ ) give better results than Shewhart median control charts (at $\lambda=1$ ) do. Similar picture about the efficiency of EWMA median charts to that we observed in EQL results, provided in Tables 1-3, can be observed for the RARL and PCI values. 


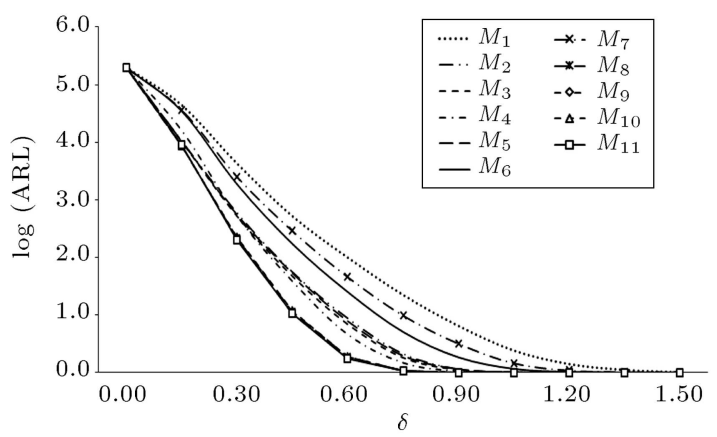

(a)

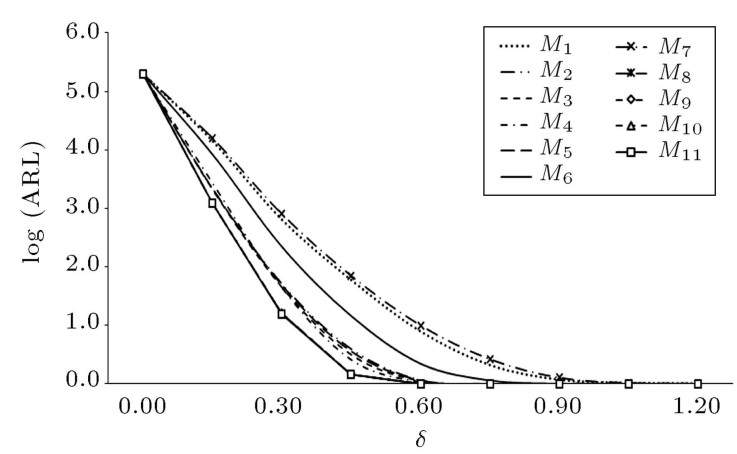

(c)

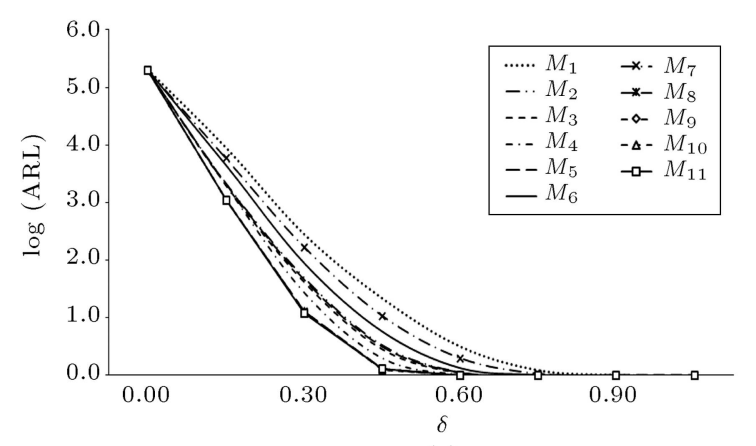

(e)

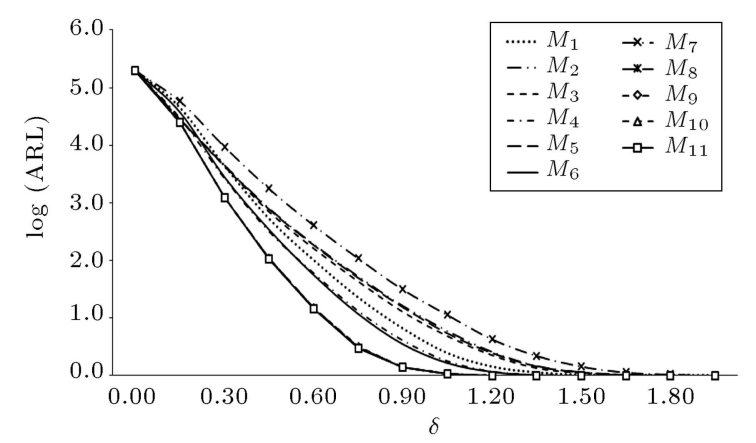

(b)

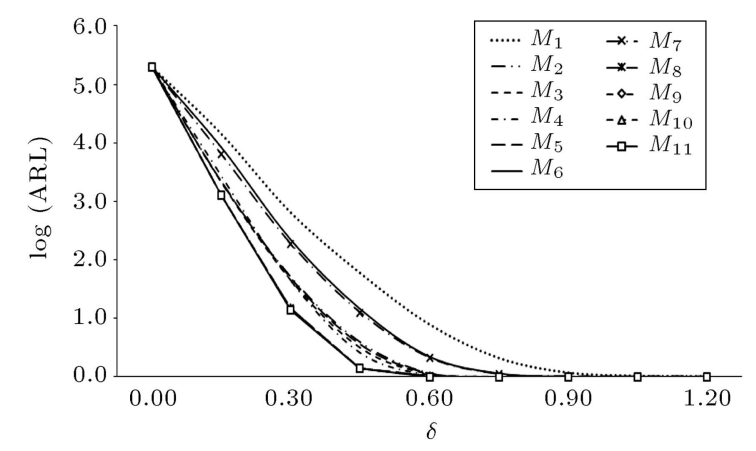

(d)

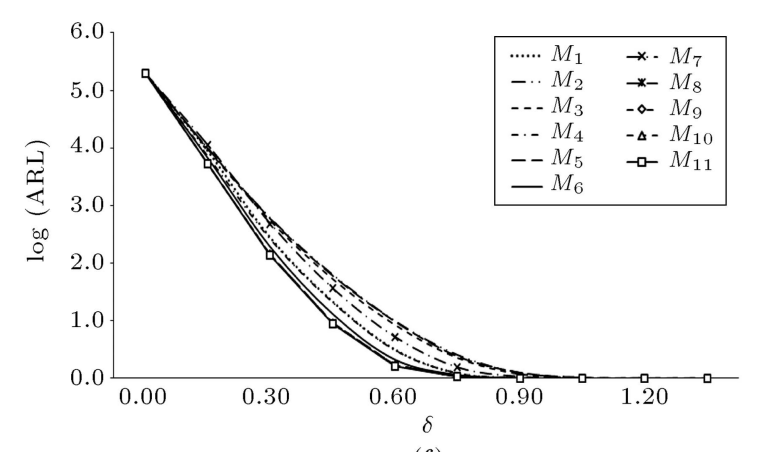

(f)

Figure 1. ARL curves for EWMA median control charts at $\mathrm{ARL}_{0}=200$, and $\lambda=0.50:$ (a) $n=5, \rho_{x y}=0.90, \rho_{y z}=0.75$, and $\rho_{z x}=0.50$; (b) $n=5, \rho_{x y}=0.60, \rho_{y z}=0.75$, and $\rho_{z x}=0.30$; (c) $n=10, \rho_{x y}=0.90, \rho_{y z}=0.90$, and $\rho_{z x}=0.90$; (d) $n=10, \rho_{x y}=0.90, \rho_{y z}=0.60$, and $\rho_{z x}=0.30 ;$ (e) $n=15, \rho_{x y}=0.90, \rho_{y z}=0.75$, and $\rho_{z x}=0.50 ;$ and (f) $n=15$, $\rho_{x y}=0.50, \rho_{y z}=0.50$, and $\rho_{z x}=0.10$.

ii. Comparison of mean control charts: We have also compared the performance of EWMA mean control charts based on the estimators $T_{i}(i=1,2, \cdots, 11)$ for the same choices $n, \rho_{x y}$, $\rho_{y z}, \rho_{z x}$, and $\lambda$ as used for EWMA median control charts using ARL curves and other measures. The ARL curves (using log (ARL) on $Y$-axis) are presented in Figure 2 while other measures are presented in Table 4 . It can be observed that the EWMA mean charts, $T_{8}-T_{11}$, which are based on two auxiliary variables, have low curves as compared to other charts (cf. Figure 2). It means that the detection ability of EWMA mean charts is enhanced due to the use of more auxiliary variables, which is also observed in the case of EWMA control charts based on median estimators. The values of EQL, RARL, and PCI presented in Table 4 also indicate the dominant performance of control charts based on $T_{8}-T_{11}$.

iii. Comparison of mean and median control charts: In order to do a cross comparison for the performance of EWMA mean and median control charts, we construct ARL curves for some selective estimators and choices of $n, \rho_{x y}, \rho_{y z}, \rho_{z x}$, and $\lambda$, which are presented in Figure 3 . It can be observed from the comparative analysis of Figure 3 that the ARL curves for EWMA mean charts are lower than the ARL curves of EWMA median charts for some selected estimators. It means that 
Table 1. EQL, RARL, and PCI for EWMA median charts with $n=5, \mathrm{ARL}_{0}=200$, and $\lambda=0.25$.

\begin{tabular}{|c|c|c|c|c|c|c|c|c|c|c|c|c|}
\hline Correlations & Performance measures & $M_{1}$ & $M_{2}$ & $M_{3}$ & $M_{4}$ & $M_{5}$ & $M_{6}$ & $M_{7}$ & $M_{8}$ & $M_{9}$ & $M_{10}$ & $M_{11}$ \\
\hline$\rho_{x y}=0.90$ & EQL & 3.27 & 3.09 & 3.08 & 3.08 & 3.09 & 3.15 & 3.30 & 3.06 & 3.06 & 3.06 & 3.06 \\
\hline$\rho_{y z}=0.90$ & RARL & 1.58 & 1.07 & 1.06 & 1.05 & 1.06 & 1.23 & 1.67 & 1.01 & 1.00 & 1.01 & 1.00 \\
\hline$\rho_{z x}=0.90$ & PCI & 1.07 & 1.01 & 1.01 & 1.01 & 1.01 & 1.03 & 1.08 & 1.00 & 1.00 & 1.00 & 1.00 \\
\hline$\rho_{x y}=0.90$ & EQL & 3.27 & 3.09 & 3.08 & 3.08 & 3.09 & 3.15 & 3.20 & 3.05 & 3.05 & 3.05 & 3.05 \\
\hline$\rho_{y z}=0.75$ & RARL & 1.66 & 1.10 & 1.09 & 1.08 & 1.09 & 1.28 & 1.43 & 1.01 & 1.00 & 1.01 & 1.00 \\
\hline$\rho_{z x}=0.50$ & PCI & 1.07 & 1.01 & 1.01 & 1.01 & 1.01 & 1.03 & 1.05 & 1.00 & 1.00 & 1.00 & 1.00 \\
\hline$\rho_{x y}=0.90$ & EQL & 3.27 & 3.09 & 3.08 & 3.08 & 3.09 & 3.15 & 3.15 & 3.06 & 3.06 & 3.06 & 3.06 \\
\hline$\rho_{y z}=0.60$ & RARL & 1.58 & 1.07 & 1.06 & 1.05 & 1.06 & 1.23 & 1.23 & 1.01 & 1.00 & 1.01 & 1.00 \\
\hline$\rho_{z x}=0.30$ & PCI & 1.07 & 1.01 & 1.01 & 1.01 & 1.01 & 1.03 & 1.03 & 1.00 & 1.00 & 1.00 & 1.00 \\
\hline$\rho_{x y}=0.50$ & EQL & 3.27 & 3.41 & 3.39 & 3.25 & 3.40 & 3.22 & 3.35 & 3.20 & 3.19 & 3.20 & 3.19 \\
\hline$\rho_{y z}=0.50$ & RARL & 1.10 & 1.30 & 1.27 & 1.07 & 1.29 & 1.04 & 1.19 & 1.01 & 1.00 & 1.01 & 1.00 \\
\hline$\rho_{z x}=0.10$ & PCI & 1.03 & 1.07 & 1.06 & 1.02 & 1.07 & 1.01 & 1.05 & 1.00 & 1.00 & 1.00 & 1.00 \\
\hline$\rho_{x y}=0.60$ & EQL & 3.27 & 3.34 & 3.31 & 3.21 & 3.33 & 3.21 & 3.52 & 3.12 & 3.12 & 3.12 & 3.12 \\
\hline$\rho_{y z}=0.75$ & RARL & 1.29 & 1.46 & 1.41 & 1.17 & 1.44 & 1.16 & 1.79 & 1.01 & 1.01 & 1.01 & 1.00 \\
\hline$\rho_{z x}=0.30$ & PCI & 1.05 & 1.07 & 1.06 & 1.03 & 1.07 & 1.03 & 1.13 & 1.00 & 1.00 & 1.00 & 1.00 \\
\hline$\rho_{x y}=0.50$ & EQL & 3.27 & 3.41 & 3.38 & 3.24 & 3.40 & 3.22 & 3.23 & 3.23 & 3.23 & 3.23 & 3.23 \\
\hline$\rho_{y z}=0.10$ & RARL & 1.04 & 1.22 & 1.18 & 1.02 & 1.21 & 1.00 & 1.01 & 1.01 & 1.00 & 1.01 & 1.00 \\
\hline$\rho_{z x}=0.10$ & PCI & 1.01 & 1.06 & 1.05 & 1.01 & 1.06 & 1.00 & 1.00 & 1.00 & 1.00 & 1.00 & 1.00 \\
\hline$\rho_{x y}=0.75$ & EQL & 3.27 & 3.21 & 3.20 & 3.15 & 3.21 & 3.18 & 3.16 & 3.15 & 3.15 & 3.15 & 3.15 \\
\hline$\rho_{y z}=0.10$ & RARL & 1.18 & 1.11 & 1.09 & 1.00 & 1.10 & 1.05 & 1.01 & 1.01 & 1.01 & 1.01 & 1.01 \\
\hline$\rho_{z x}=0.10$ & PCI & 1.04 & 1.02 & 1.02 & 1.00 & 1.02 & 1.01 & 1.00 & 1.00 & 1.00 & 1.00 & 1.00 \\
\hline
\end{tabular}

the performance of EWMA median charts is not better than that of EWMA mean charts under the uncontaminated process environment. Due to this reason, we have compared both the EWMA median and mean charts under the contaminated process environment in the following section.

\subsection{Comparison of control charts under contaminated environments}

In this section, we have considered an uncontaminated process environment and three contaminated process environments in order to compare the robustness of proposed EWMA median charts. Without loss of generality, we have used $\mu_{0}=5$ and $\sigma_{0}^{2}=1$ as uncontaminated process environment throughout the study so far, which is denoted as $\left(E_{1}\right)$ here. To study the robustness of EWMA median and mean charts, we have considered three contaminated process environments, namely, $E_{2}, E_{3}$, and $E_{4}$, with certain percentages of diffused outliers. The description of these process environments is as follows:

- $E_{1}:(1-\alpha) 100 \%$ data from $\mu_{y}=6, \mu_{x}=\mu_{z}=5$ and $\sigma_{y}^{2}=\sigma_{x}^{2}=\sigma_{z}^{2}=1$;

- $E_{2}:(1-\alpha) 100 \%$ data from $\mu_{y}=\mu_{x}=\mu_{z}=5$ and $\sigma_{y}^{2}=\sigma_{x}^{2}=\sigma_{z}^{2}=1$ and $(\alpha) 100 \%$ observations from $\mu_{y}=\mu_{x}=\mu_{z}=6$ and $\sigma_{y}^{2}=\sigma_{x}^{2}=\sigma_{z}^{2}=1$;

- $E_{3}$ : $(1-\alpha) 100 \%$ observations from $\mu_{y}=\mu_{x}=$ 
Table 2. EQL, RARL, and PCI for EWMA median charts with $n=10$ and $\mathrm{ARL}_{0}=200$.

\begin{tabular}{|c|c|c|c|c|c|c|c|c|c|c|c|c|}
\hline \multirow[b]{2}{*}{$\lambda$} & & \multicolumn{11}{|c|}{$\rho_{x y}=0.90, \rho_{y z}=0.90, \rho_{z x}=0.90$} \\
\hline & & $M_{1}$ & $M_{2}$ & $M_{3}$ & $M_{4}$ & $M_{5}$ & $M_{6}$ & $M_{7}$ & $M_{8}$ & $M_{9}$ & $M_{10}$ & $M_{11}$ \\
\hline \multirow{3}{*}{1.0} & EQL & 3.96 & 3.28 & 3.26 & 3.30 & 3.27 & 3.59 & 4.06 & 3.19 & 3.19 & 3.19 & 3.19 \\
\hline & RARL & 1.86 & 1.11 & 1.09 & 1.12 & 1.11 & 1.44 & 1.97 & 1.00 & 1.00 & 1.00 & 1.00 \\
\hline & PCI & 1.24 & 1.03 & 1.02 & 1.03 & 1.03 & 1.13 & 1.27 & 1.00 & 1.00 & 1.00 & 1.00 \\
\hline \multirow{3}{*}{0.75} & EQL & 3.50 & 3.15 & 3.14 & 3.15 & 3.15 & 3.30 & 3.55 & 3.10 & 3.10 & 3.10 & 3.10 \\
\hline & RARL & 1.69 & 1.09 & 1.08 & 1.08 & 1.08 & 1.34 & 1.78 & 1.00 & 1.00 & 1.00 & 1.00 \\
\hline & PCI & 1.13 & 1.02 & 1.01 & 1.02 & 1.01 & 1.07 & 1.15 & 1.00 & 1.00 & 1.00 & 1.00 \\
\hline \multirow{3}{*}{0.50} & EQL & 3.25 & 3.08 & 3.07 & 3.08 & 3.08 & 3.15 & 3.27 & 3.05 & 3.05 & 3.05 & 3.05 \\
\hline & RARL & 1.56 & 1.07 & 1.06 & 1.06 & 1.06 & 1.27 & 1.62 & 1.00 & 1.00 & 1.00 & 1.00 \\
\hline & PCI & 1.06 & 1.01 & 1.01 & 1.01 & 1.01 & 1.03 & 1.07 & 1.00 & 1.00 & 1.00 & 1.00 \\
\hline \multirow{3}{*}{0.25} & EQL & 3.10 & 3.03 & 3.03 & 3.03 & 3.03 & 3.06 & 3.10 & 3.02 & 3.02 & 3.02 & 3.02 \\
\hline & RARL & 1.60 & 1.09 & 1.08 & 1.07 & 1.09 & 1.29 & 1.64 & 1.01 & 1.00 & 1.01 & 1.00 \\
\hline & PCI & 1.03 & 1.00 & 1.00 & 1.00 & 1.00 & 1.01 & 1.03 & 1.00 & 1.00 & 1.00 & 1.00 \\
\hline & & \multicolumn{11}{|c|}{$\rho_{x y}=0.75, \rho_{y z}=0.10, \rho_{z x}=0.10$} \\
\hline$\lambda$ & & $M_{1}$ & $M_{2}$ & $M_{3}$ & $M_{4}$ & $M_{5}$ & $M_{6}$ & $M_{7}$ & $M_{8}$ & $M_{9}$ & $M_{10}$ & $M_{11}$ \\
\hline \multirow{3}{*}{1.0} & EQL & 3.96 & 3.70 & 3.65 & 3.52 & 3.68 & 3.69 & 3.53 & 3.53 & 3.53 & 3.53 & 3.53 \\
\hline & RARL & 1.24 & 1.10 & 1.08 & 1.00 & 1.09 & 1.09 & 1.00 & 1.00 & 1.00 & 1.00 & 1.00 \\
\hline & PCI & 1.12 & 1.05 & 1.04 & 1.00 & 1.05 & 1.05 & 1.00 & 1.00 & 1.00 & 1.00 & 1.00 \\
\hline \multirow{3}{*}{0.75} & EQL & 3.50 & 3.37 & 3.35 & 3.27 & 3.36 & 3.36 & 3.27 & 3.27 & 3.27 & 3.27 & 3.27 \\
\hline & RARL & 1.20 & 1.09 & 1.07 & 1.00 & 1.08 & 1.07 & 1.00 & 1.00 & 1.00 & 1.00 & 1.00 \\
\hline & PCI & 1.07 & 1.03 & 1.02 & 1.00 & 1.03 & 1.03 & 1.00 & 1.00 & 1.00 & 1.00 & 1.00 \\
\hline \multirow{3}{*}{0.50} & EQL & 3.25 & 3.18 & 3.18 & 3.14 & 3.18 & 3.17 & 3.14 & 3.14 & 3.14 & 3.14 & 3.14 \\
\hline & RARL & 1.16 & 1.07 & 1.06 & 1.00 & 1.07 & 1.05 & 1.00 & 1.00 & 1.00 & 1.00 & 1.00 \\
\hline & PCI & 1.04 & 1.02 & 1.01 & 1.00 & 1.01 & 1.01 & 1.00 & 1.00 & 1.00 & 1.00 & 1.00 \\
\hline \multirow{3}{*}{0.25} & EQL & 3.10 & 3.08 & 3.07 & 3.06 & 3.07 & 3.07 & 3.06 & 3.06 & 3.06 & 3.06 & 3.06 \\
\hline & RARL & 1.10 & 1.05 & 1.04 & 1.00 & 1.04 & 1.03 & 1.00 & 1.00 & 1.00 & 1.00 & 1.00 \\
\hline & PCI & 1.01 & 1.01 & 1.01 & 1.00 & 1.01 & 1.00 & 1.00 & 1.00 & 1.00 & 1.00 & 1.00 \\
\hline & & \multicolumn{11}{|c|}{$\rho_{x y}=0.90, \rho_{y z}=0.60, \rho_{z x}=0.30$} \\
\hline$\lambda$ & & $M_{1}$ & $M_{2}$ & $M_{3}$ & $M_{4}$ & $M_{5}$ & $M_{6}$ & $M_{7}$ & $M_{8}$ & $M_{9}$ & $M_{10}$ & $M_{11}$ \\
\hline \multirow{3}{*}{1.0} & EQL & 3.96 & 3.28 & 3.26 & 3.30 & 3.27 & 3.59 & 3.52 & 3.19 & 3.19 & 3.19 & 3.20 \\
\hline & RARL & 1.85 & 1.11 & 1.09 & 1.11 & 1.10 & 1.43 & 1.37 & 1.00 & 1.00 & 1.00 & 1.00 \\
\hline & PCI & 1.24 & 1.03 & 1.02 & 1.03 & 1.02 & 1.12 & 1.10 & 1.00 & 1.00 & 1.00 & 1.00 \\
\hline \multirow{3}{*}{0.75} & EQL & 3.50 & 3.15 & 3.14 & 3.15 & 3.15 & 3.30 & 3.27 & 3.10 & 3.10 & 3.10 & 3.10 \\
\hline & RARL & 1.69 & 1.09 & 1.07 & 1.08 & 1.08 & 1.34 & 1.30 & 1.00 & 1.00 & 1.00 & 1.00 \\
\hline & PCI & 1.13 & 1.01 & 1.01 & 1.02 & 1.01 & 1.06 & 1.05 & 1.00 & 1.00 & 1.00 & 1.00 \\
\hline \multirow{3}{*}{0.50} & EQL & 3.25 & 3.08 & 3.07 & 3.08 & 3.08 & 3.15 & 3.14 & 3.05 & 3.05 & 3.05 & 3.05 \\
\hline & RARL & 1.56 & 1.07 & 1.06 & 1.06 & 1.06 & 1.27 & 1.24 & 1.00 & 1.00 & 1.00 & 1.00 \\
\hline & PCI & 1.06 & 1.01 & 1.01 & 1.01 & 1.01 & 1.03 & 1.03 & 1.00 & 1.00 & 1.00 & 1.00 \\
\hline \multirow{3}{*}{0.25} & EQL & 3.10 & 3.03 & 3.03 & 3.03 & 3.03 & 3.06 & 3.06 & 3.02 & 3.02 & 3.02 & 3.02 \\
\hline & RARL & 1.63 & 1.10 & 1.09 & 1.07 & 1.10 & 1.31 & 1.29 & 1.01 & 1.00 & 1.01 & 1.00 \\
\hline & PCI & 1.03 & 1.00 & 1.00 & 1.00 & 1.00 & 1.01 & 1.01 & 1.00 & 1.00 & 1.00 & 1.00 \\
\hline
\end{tabular}


Table 3. EQL, RARL, and PCI for EWMA median charts with $n=15$ and $\mathrm{ARL}_{0}=200$.

\begin{tabular}{|c|c|c|c|c|c|c|c|c|c|c|c|c|}
\hline \multirow[b]{2}{*}{$\lambda$} & & \multicolumn{11}{|c|}{$\rho_{x y}=0.50, \rho_{y z}=0.50, \rho_{z x}=0.10$} \\
\hline & & $M_{1}$ & $M_{2}$ & $M_{3}$ & $M_{4}$ & $M_{5}$ & $M_{6}$ & $M_{7}$ & $M_{8}$ & $M_{9}$ & $M_{10}$ & $M_{11}$ \\
\hline \multirow{3}{*}{1.0} & EQL & 3.62 & 3.92 & 3.87 & 3.58 & 3.90 & 3.54 & 3.78 & 3.45 & 3.45 & 3.45 & 3.45 \\
\hline & RARL & 1.11 & 1.30 & 1.27 & 1.08 & 1.29 & 1.05 & 1.21 & 1.00 & 1.00 & 1.00 & 1.00 \\
\hline & PCI & 1.05 & 1.14 & 1.12 & 1.04 & 1.13 & 1.02 & 1.10 & 1.00 & 1.00 & 1.00 & 1.00 \\
\hline \multirow[b]{2}{*}{0.75} & EQL & 3.33 & 3.49 & 3.46 & 3.31 & 3.48 & 3.28 & 3.41 & 3.24 & 3.24 & 3.24 & 3.24 \\
\hline & RARL & 1.09 & 1.25 & 1.22 & 1.07 & 1.24 & 1.04 & 1.17 & 1.00 & 1.00 & 1.00 & 1.00 \\
\hline \multirow{4}{*}{0.50} & PCI & 1.03 & 1.08 & 1.07 & 1.02 & 1.07 & 1.01 & 1.05 & 1.00 & 1.00 & 1.00 & 1.00 \\
\hline & EQL & 3.17 & 3.24 & 3.23 & 3.16 & 3.24 & 3.14 & 3.21 & 3.12 & 3.12 & 3.12 & 3.12 \\
\hline & RARL & 1.07 & 1.21 & 1.18 & 1.06 & 1.20 & 1.03 & 1.14 & 1.00 & 1.00 & 1.00 & 1.00 \\
\hline & PCI & 1.02 & 1.04 & 1.04 & 1.01 & 1.04 & 1.01 & 1.03 & 1.00 & 1.00 & 1.00 & 1.00 \\
\hline \multirow{3}{*}{0.25} & EQL & 3.07 & 3.10 & 3.09 & 3.07 & 3.10 & 3.06 & 3.08 & 3.05 & 3.05 & 3.05 & 3.05 \\
\hline & RARL & 1.04 & 1.13 & 1.11 & 1.04 & 1.12 & 1.02 & 1.08 & 1.00 & 1.00 & 1.00 & 1.00 \\
\hline & PCI & 1.01 & 1.02 & 1.01 & 1.00 & 1.01 & 1.00 & 1.01 & 1.00 & 1.00 & 1.00 & 1.00 \\
\hline & & \multicolumn{11}{|c|}{$\rho_{x y}=0.90, \rho_{y z}=0.75, \rho_{z x}=0.50$} \\
\hline$\lambda$ & & $M_{1}$ & $M_{2}$ & $M_{3}$ & $M_{4}$ & $M_{5}$ & $M_{6}$ & $M_{7}$ & $M_{8}$ & $M_{9}$ & $M_{10}$ & $M_{11}$ \\
\hline \multirow{3}{*}{1.0} & EQL & 3.62 & 3.27 & 3.26 & 3.24 & 3.26 & 3.40 & 3.49 & 3.18 & 3.18 & 3.18 & 3.18 \\
\hline & RARL & 1.53 & 1.12 & 1.10 & 1.07 & 1.11 & 1.26 & 1.37 & 1.00 & 1.00 & 1.00 & 1.00 \\
\hline & PCI & 1.14 & 1.03 & 1.03 & 1.02 & 1.03 & 1.07 & 1.10 & 1.00 & 1.00 & 1.00 & 1.00 \\
\hline \multirow{3}{*}{0.75} & EQL & 3.33 & 3.15 & 3.14 & 3.13 & 3.15 & 3.21 & 3.26 & 3.10 & 3.09 & 3.10 & 3.09 \\
\hline & RARL & 1.44 & 1.11 & 1.09 & 1.06 & 1.10 & 1.20 & 1.31 & 1.00 & 1.00 & 1.00 & 1.00 \\
\hline & PCI & 1.08 & 1.02 & 1.01 & 1.01 & 1.02 & 1.04 & 1.05 & 1.00 & 1.00 & 1.00 & 1.00 \\
\hline \multirow{3}{*}{0.50} & EQL & 3.17 & 3.07 & 3.07 & 3.06 & 3.07 & 3.10 & 3.13 & 3.05 & 3.05 & 3.05 & 3.05 \\
\hline & RARL & 1.36 & 1.08 & 1.07 & 1.04 & 1.08 & 1.16 & 1.24 & 1.00 & 1.00 & 1.00 & 1.00 \\
\hline & PCI & 1.04 & 1.01 & 1.01 & 1.01 & 1.01 & 1.02 & 1.03 & 1.00 & 1.00 & 1.00 & 1.00 \\
\hline \multirow{3}{*}{0.25} & EQL & 3.07 & 3.03 & 3.03 & 3.03 & 3.03 & 3.04 & 3.06 & 3.02 & 3.02 & 3.02 & 3.02 \\
\hline & RARL & 1.43 & 1.11 & 1.09 & 1.05 & 1.10 & 1.19 & 1.31 & 1.01 & 1.00 & 1.00 & 1.00 \\
\hline & PCI & 1.02 & 1.00 & 1.00 & 1.00 & 1.00 & 1.01 & 1.01 & 1.00 & 1.00 & 1.00 & 1.00 \\
\hline & & \multicolumn{11}{|c|}{$\rho_{x y}=0.50, \rho_{y z}=0.10, \rho_{z x}=0.10$} \\
\hline$\lambda$ & & $M_{1}$ & $M_{2}$ & $M_{3}$ & $M_{4}$ & $M_{5}$ & $M_{6}$ & $M_{7}$ & $M_{8}$ & $M_{9}$ & $M_{10}$ & $M_{11}$ \\
\hline \multirow{3}{*}{1.0} & EQL & 3.62 & 3.92 & 3.87 & 3.58 & 3.90 & 3.54 & 3.54 & 3.53 & 3.54 & 3.53 & 3.53 \\
\hline & RARL & 1.05 & 1.22 & 1.20 & 1.03 & 1.21 & 1.00 & 1.00 & 1.00 & 1.00 & 1.00 & 1.00 \\
\hline & PCI & 1.03 & 1.11 & 1.10 & 1.01 & 1.10 & 1.00 & 1.00 & 1.00 & 1.00 & 1.00 & 1.00 \\
\hline \multirow{3}{*}{0.75} & EQL & 3.33 & 3.49 & 3.46 & 3.31 & 3.48 & 3.28 & 3.28 & 3.28 & 3.28 & 3.28 & 3.28 \\
\hline & RARL & 1.04 & 1.18 & 1.16 & 1.03 & 1.17 & 1.00 & 1.00 & 1.00 & 1.00 & 1.00 & 1.00 \\
\hline & PCI & 1.02 & 1.06 & 1.05 & 1.01 & 1.06 & 1.00 & 1.00 & 1.00 & 1.00 & 1.00 & 1.00 \\
\hline \multirow{3}{*}{0.50} & EQL & 3.17 & 3.24 & 3.23 & 3.16 & 3.24 & 3.14 & 3.14 & 3.14 & 3.14 & 3.14 & 3.14 \\
\hline & RARL & 1.04 & 1.16 & 1.14 & 1.03 & 1.15 & 1.00 & 1.00 & 1.00 & 1.00 & 1.00 & 1.00 \\
\hline & PCI & 1.01 & 1.03 & 1.03 & 1.01 & 1.03 & 1.00 & 1.00 & 1.00 & 1.00 & 1.00 & 1.00 \\
\hline \multirow{3}{*}{0.25} & EQL & 3.07 & 3.10 & 3.09 & 3.07 & 3.10 & 3.06 & 3.06 & 3.06 & 3.06 & 3.06 & 3.06 \\
\hline & RARL & 1.02 & 1.10 & 1.08 & 1.02 & 1.09 & 1.00 & 1.00 & 1.00 & 1.00 & 1.00 & 1.00 \\
\hline & PCI & 1.00 & 1.01 & 1.01 & 1.00 & 1.01 & 1.00 & 1.00 & 1.00 & 1.00 & 1.00 & 1.00 \\
\hline
\end{tabular}




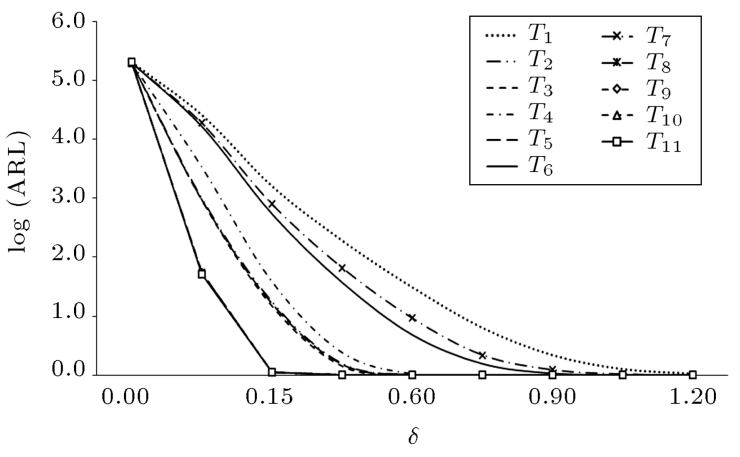

(a)

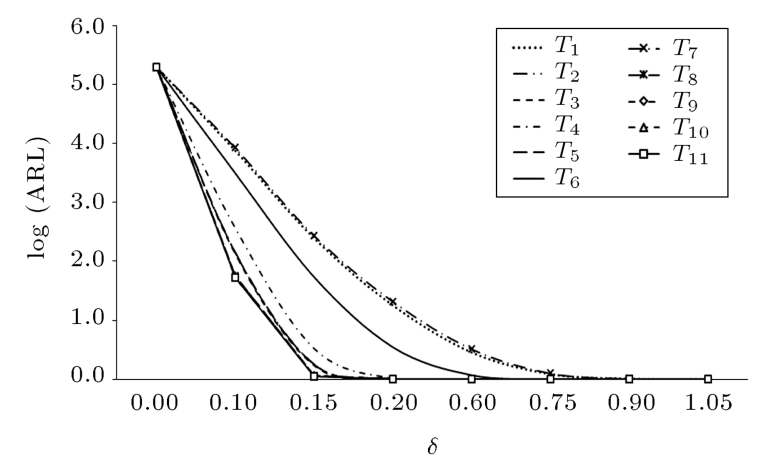

(c)

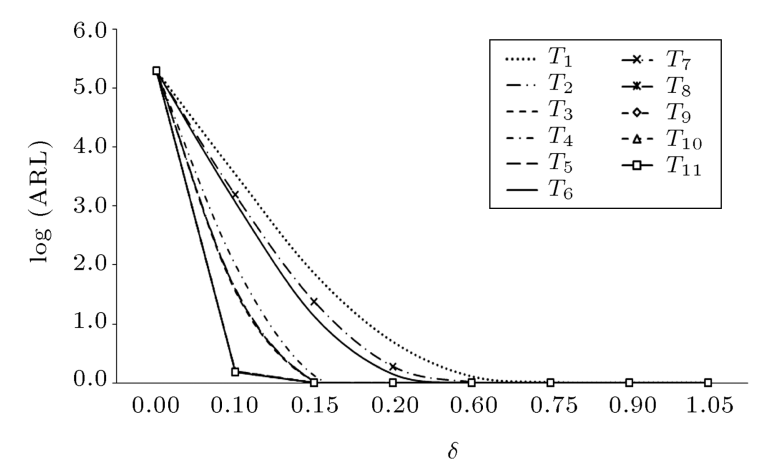

(e)

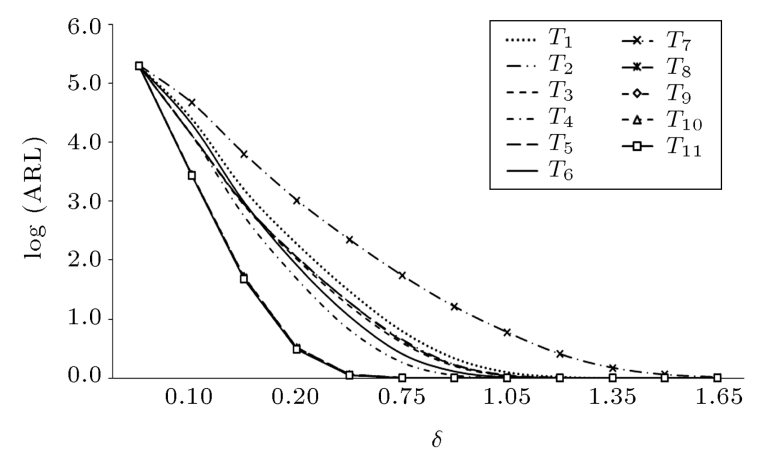

(b)

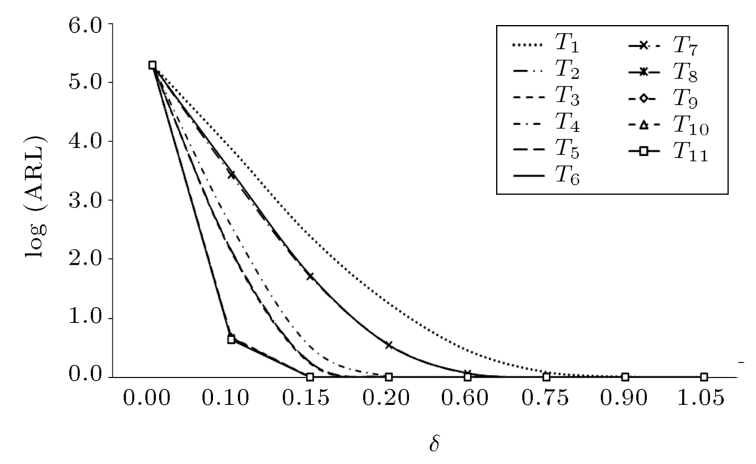

(d)

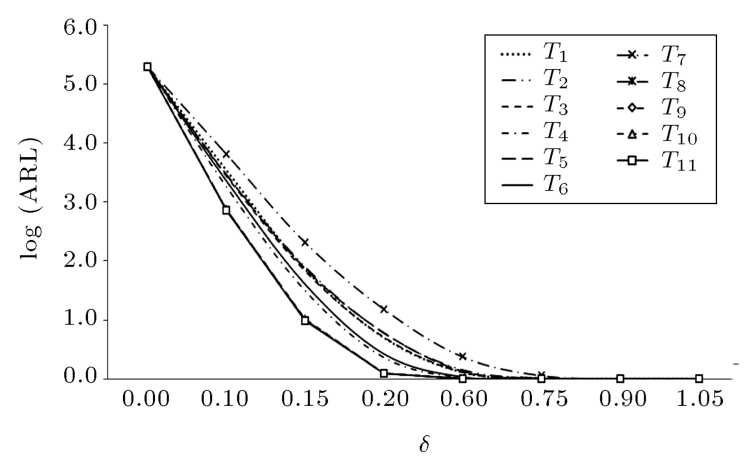

(f)

Figure 2. ARL curves for EWMA mean control charts at $\mathrm{ARL}_{0}=200$, and $\lambda=0.50$ : (a) $n=5, \rho_{x y}=0.90, \rho_{y z}=0.75$, and $\rho_{z x}=0.50$; (b) $n=5, \rho_{x y}=0.60, \rho_{y z}=0.75$, and $\rho_{z x}=0.30 ;$ (c) $n=10, \rho_{x y}=0.90, \rho_{y z}=0.90$, and $\rho_{z x}=0.90 ;(d)$ $n=10, \rho_{x y}=0.90, \rho_{y z}=0.60$, and $\rho_{z x}=0.30 ;$ (e) $n=15, \rho_{x y}=0.90, \rho_{y z}=0.75$, and $\rho_{z x}=0.50 ;$ and (f) $n=15$, $\rho_{x y}=0.50, \rho_{y z}=0.50$, and $\rho_{z x}=0.10$.

$\mu_{z}=5$ and $\sigma_{y}^{2}=2, \sigma_{x}^{2}=\sigma_{z}^{2}=1$ and $(\alpha) 100 \%$ observations from $\mu_{y}=\mu_{x}=\mu_{z}=5$ and $\sigma_{y}^{2}=\sigma_{x}^{2}=$ $\sigma_{z}^{2}=2$;

- $E_{4}:(1-\alpha) 100 \%$ observations from $\mu_{y}=\mu_{x}=\mu_{z}=$ 5 and $\sigma_{y}^{2}=\sigma_{x}^{2}=\sigma_{z}^{2}=1$ and $(\alpha) 100 \%$ observations from $\mu_{y}=6, \mu_{x}=\mu_{z}=5$ and $\sigma_{y}^{2}=2, \sigma_{x}^{2}=\sigma_{z}^{2}=1$.

Here, we examine two types of contamination effects on $\mathrm{ARL}$, namely, $\mathrm{ARL}_{0}$ contamination effect (examined relative to the corresponding $\mathrm{ARL}_{0}$ values) and $\mathrm{ARL}_{1}$ contamination effect (studied by adjusting the control limits for contamination effects to fix $\left.\mathrm{ARL}_{0}=200\right)$. For this purpose, we have generated data from normally distributed process environments
$\left(E_{1}-E_{4}\right)$ for $n=5,10,15$ and $\lambda=0.5$ at selected choices of $\rho_{x y}, \rho_{y z}, \rho_{z x}$, and presented it in the form of bar charts in Figures 4-7. Figures 4 and 5 represent the $\mathrm{ARL}_{0}$ contamination effect of all EWMA median and mean charts, respectively, relative to $\mathrm{ARL}_{0}=200$, while Figures 6 and 7 explain $\mathrm{ARL}_{1}$ contamination effect at only two selective values of shifts (i.e., $\delta=0.10$ and 0.15).

From Figure 4, it can be observed that the contaminated $\mathrm{ARL}_{0}$ bars for the EWMA median charts under the contaminated environments $\left(E_{2}-E_{4}\right)$ are relatively closer to the $\mathrm{ARL}_{0}$ bars under the uncontaminated environment $E_{1}$ than the bar charts for EWMA mean charts, provided in Figure 5, are. It 
Table 4. EQL, RARL, and PCI for EWMA mean charts $\mathrm{ARL}_{0}=200$ and $\lambda=0.50$.

\begin{tabular}{|c|c|c|c|c|c|c|c|c|c|c|c|c|}
\hline Correlations & & $T_{1}$ & $T_{2}$ & $T_{3}$ & $T_{4}$ & $T_{5}$ & $T_{6}$ & $T_{7}$ & $T_{8}$ & $T_{9}$ & $T_{10}$ & $T_{11}$ \\
\hline & & \multicolumn{11}{|c|}{$n=5$} \\
\hline$\rho_{x y}=0.90$ & EQL & 3.43 & 3.05 & 3.05 & 3.08 & 3.05 & 3.23 & 3.27 & 3.02 & 3.02 & 3.02 & 3.02 \\
\hline$\rho_{y z}=0.75$ & RARL & 3.81 & 1.32 & 1.30 & 1.58 & 1.31 & 2.75 & 3.00 & 1.00 & 1.00 & 1.00 & 1.00 \\
\hline$\rho_{z x}=0.50$ & $\mathrm{PCI}$ & 1.14 & 1.01 & 1.01 & 1.02 & 1.01 & 1.07 & 1.08 & 1.00 & 1.00 & 1.00 & 1.00 \\
\hline$\rho_{x y}=0.60$ & EQL & 3.43 & 3.32 & 3.30 & 3.23 & 3.32 & 3.30 & 4.05 & 3.08 & 3.08 & 3.08 & 3.08 \\
\hline$\rho_{y z}=0.75$ & RARL & 1.77 & 1.56 & 1.51 & 1.34 & 1.54 & 1.48 & 2.97 & 1.01 & 1.00 & 1.01 & 1.00 \\
\hline$\rho_{z x}=0.30$ & PCI & 1.11 & 1.08 & 1.07 & 1.05 & 1.08 & 1.07 & 1.32 & 1.00 & 1.00 & 1.00 & 1.00 \\
\hline$\rho_{x y}=0.75$ & EQL & 3.43 & 3.16 & 3.15 & 3.14 & 3.16 & 3.26 & 3.14 & 3.14 & 3.14 & 3.14 & 3.14 \\
\hline$\rho_{y z}=0.10$ & RARL & 1.42 & 1.04 & 1.03 & 1.01 & 1.04 & 1.17 & 1.00 & 1.00 & 1.00 & 1.00 & 1.00 \\
\hline \multirow{2}{*}{$\rho_{z x}=0.10$} & PCI & 1.09 & 1.01 & 1.00 & 1.00 & 1.01 & 1.04 & 1.00 & 1.00 & 1.00 & 1.00 & 1.00 \\
\hline & & \multicolumn{11}{|c|}{$n=10$} \\
\hline$\rho_{x y}=0.90$ & EQL & 3.15 & 3.02 & 3.02 & 3.03 & 3.02 & 3.09 & 3.17 & 3.02 & 3.02 & 3.02 & 3.02 \\
\hline$\rho_{y z}=0.90$ & RARL & 2.13 & 1.05 & 1.04 & 1.13 & 1.05 & 1.63 & 2.22 & 1.00 & 1.00 & 1.00 & 1.00 \\
\hline$\rho_{z x}=0.90$ & PCI & 1.05 & 1.00 & 1.00 & 1.00 & 1.00 & 1.02 & 1.05 & 1.00 & 1.00 & 1.00 & 1.00 \\
\hline$\rho_{x y}=0.90$ & EQL & 3.15 & 3.02 & 3.02 & 3.03 & 3.02 & 3.09 & 3.08 & 3.01 & 3.01 & 3.01 & 3.01 \\
\hline$\rho_{y z}=0.60$ & RARL & 3.07 & 1.21 & 1.20 & 1.37 & 1.21 & 2.27 & 2.18 & 1.00 & 1.00 & 1.00 & 1.00 \\
\hline$\rho_{z x}=0.30$ & PCI & 1.05 & 1.00 & 1.00 & 1.01 & 1.00 & 1.02 & 1.02 & 1.00 & 1.00 & 1.00 & 1.00 \\
\hline$\rho_{x y}=0.75$ & EQL & 3.15 & 3.06 & 3.06 & 3.06 & 3.06 & 3.10 & 3.06 & 3.06 & 3.06 & 3.06 & 3.06 \\
\hline$\rho_{y z}=0.10$ & RARL & 1.27 & 1.03 & 1.02 & 1.01 & 1.02 & 1.11 & 1.00 & 1.00 & 1.00 & 1.00 & 1.00 \\
\hline \multirow[t]{2}{*}{$\rho_{z x}=0.10$} & PCI & 1.03 & 1.00 & 1.00 & 1.00 & 1.00 & 1.01 & 1.00 & 1.00 & 1.00 & 1.00 & 1.00 \\
\hline & & \multicolumn{11}{|c|}{$n=15$} \\
\hline$\rho_{x y}=0.90$ & EQL & 3.09 & 3.02 & 3.02 & 3.02 & 3.02 & 3.05 & 3.06 & 3.01 & 3.01 & 3.01 & 3.01 \\
\hline$\rho_{y z}=0.75$ & RARL & 2.79 & 1.17 & 1.16 & 1.30 & 1.16 & 2.04 & 2.20 & 1.00 & 1.00 & 1.00 & 1.00 \\
\hline$\rho_{z x}=0.50$ & PCI & 1.03 & 1.00 & 1.00 & 1.00 & 1.00 & 1.01 & 1.02 & 1.00 & 1.00 & 1.00 & 1.00 \\
\hline$\rho_{x y}=0.50$ & EQL & 3.09 & 3.09 & 3.09 & 3.07 & 3.09 & 3.07 & 3.14 & 3.04 & 3.04 & 3.04 & 3.04 \\
\hline$\rho_{y z}=0.50$ & RARL & 1.15 & 1.16 & 1.14 & 1.07 & 1.15 & 1.09 & 1.31 & 1.00 & 1.00 & 1.00 & 1.00 \\
\hline$\rho_{z x}=0.10$ & PCI & 1.02 & 1.02 & 1.01 & 1.01 & 1.02 & 1.01 & 1.03 & 1.00 & 1.00 & 1.00 & 1.00 \\
\hline$\rho_{x y}=0.50$ & EQL & 3.09 & 3.09 & 3.09 & 3.07 & 3.09 & 3.07 & 3.06 & 3.06 & 3.06 & 3.06 & 3.06 \\
\hline$\rho_{y z}=0.10$ & RARL & 1.07 & 1.07 & 1.06 & 1.00 & 1.07 & 1.02 & 1.00 & 1.00 & 1.00 & 1.00 & 1.00 \\
\hline$\rho_{z x}=0.10$ & PCI & 1.01 & 1.01 & 1.01 & 1.00 & 1.01 & 1.00 & 1.00 & 1.00 & 1.00 & 1.00 & 1.00 \\
\hline
\end{tabular}

means that the EWMA median charts have shown reasonably more resistance against outliers for $\mathrm{ARL}_{0}$ contamination than EWMA mean charts have done.

As well as concern about $\mathrm{ARL}_{1}$ contamination, the EWMA median charts show relatively better resis- tance ability than EWMA mean charts do, which can be seen in Figures 6 and 7 .

From these figures, we can observe that median control charts are more robust than mean control charts are. 


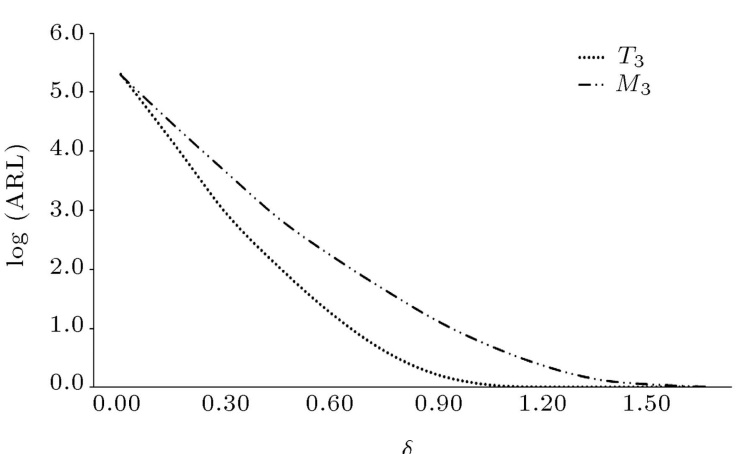

(a)

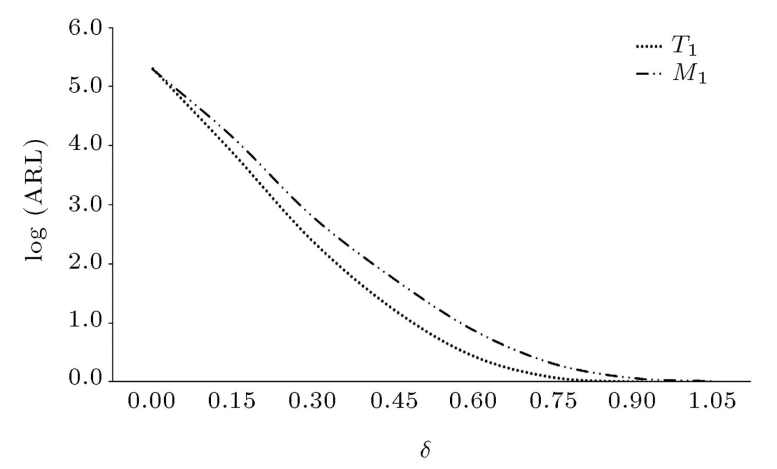

(c)

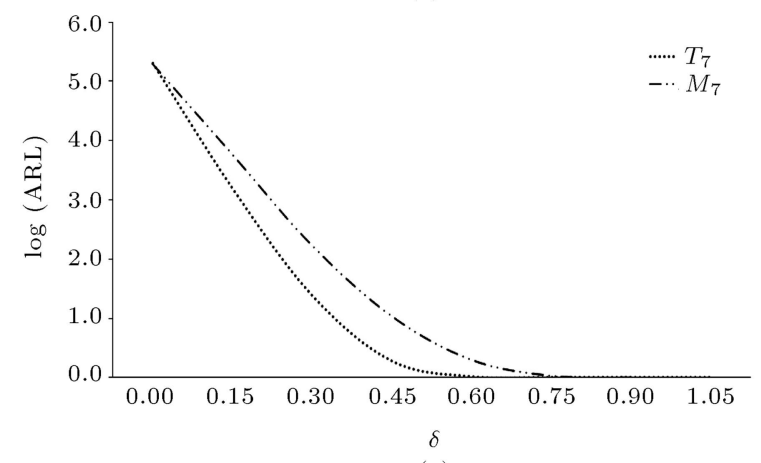

(e)

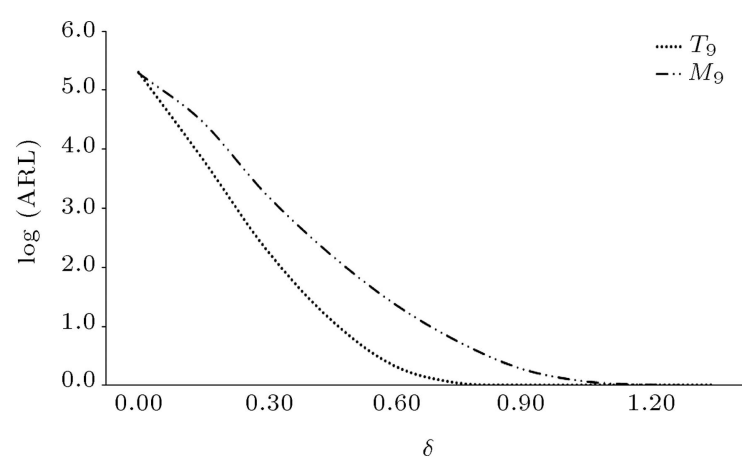

(b)

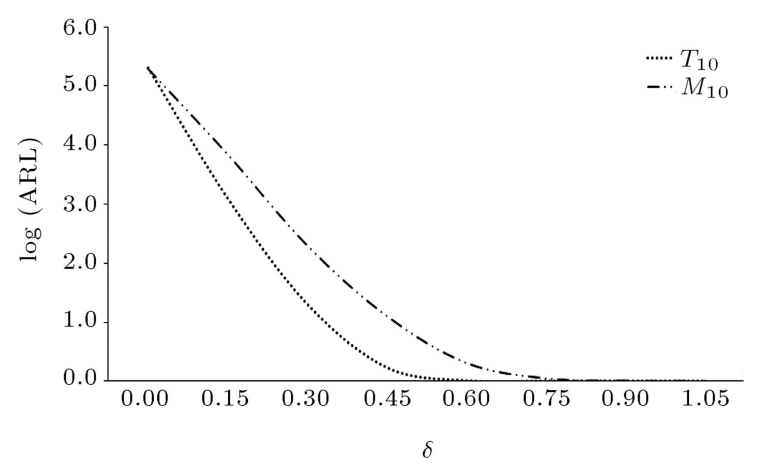

(d)

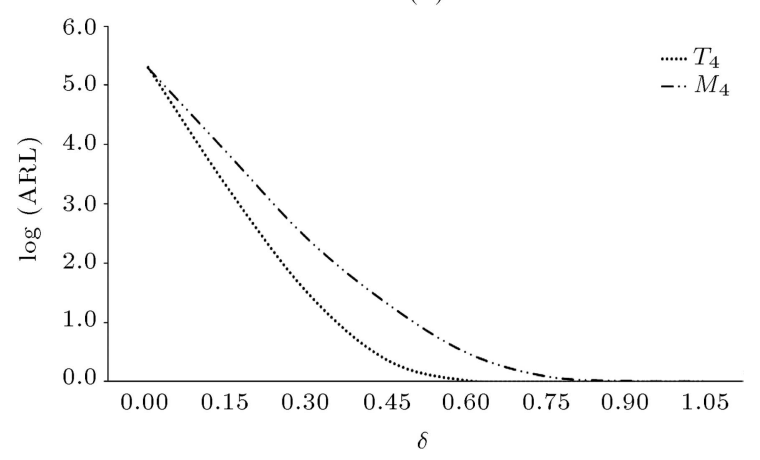

(f)

Figure 3. ARL curves for median versus mean control charts at $\mathrm{ARL}_{0}=200$, and $\lambda=0.50$ : (a) $n=5, \rho_{x y}=0.60$, $\rho_{y z}=0.75$, and $\rho_{z x}=0.30$; (b) $n=5, \rho_{x y}=0.75, \rho_{y z}=0.10$, and $\rho_{z x}=0.10$; (c) $n=10, \rho_{x y}=0.90, \rho_{y z}=0.90$, and $\rho_{z x}=0.90 ;(\mathrm{d}) n=10, \rho_{x y}=0.75, \rho_{y z}=0.10$, and $\rho_{z x}=0.10 ;$ (e) $n=15, \rho_{x y}=0.90, \rho_{y z}=0.75$, and $\rho_{z x}=0.50$; and (f) $n=15, \rho_{x y}=0.50, \rho_{y z}=0.50$, and $\rho_{z x}=0.10$

\section{Illustrative example}

In this section, we provide a descriptive example as well as a case study to compare the performance of control charts. Through these illustrative applications of the control structure, the importance of effective changes detection in parameters of the process using median based control charts is highlighted. In both cases, we select three charts representing study variable, $Y$, first auxiliary variable, $X$, and second auxiliary variable, $Z$. The variables $Y, X$, and $Z$ can represent different real life applications; for example:

(i) In process monitoring of missile testing, we may consider the efficiency of projectile as $Y$, its momentum as $X$, and the weight of carrier as $Z$; (ii) In process monitoring of nuclear power generation, the amount of power generated can be taken as $Y$, the half-life of fissile material as $X$, and the amount of fuel as $Z$;

(iii) In measuring the resistance of wire, we may take resistance as $Y$, the width of wire as $X$, and current supplied as $Z$;

(iv) In chemical reactions, the rate of reaction can be considered as $Y$, the activation energy of molecules as $X$, and the stoichiometric amount of reaction as $Z$;

(v) In materials sample sintering in partial gas pressure, different diffusion of gas into a furnace chamber can be taken as $Y$, the area of the 


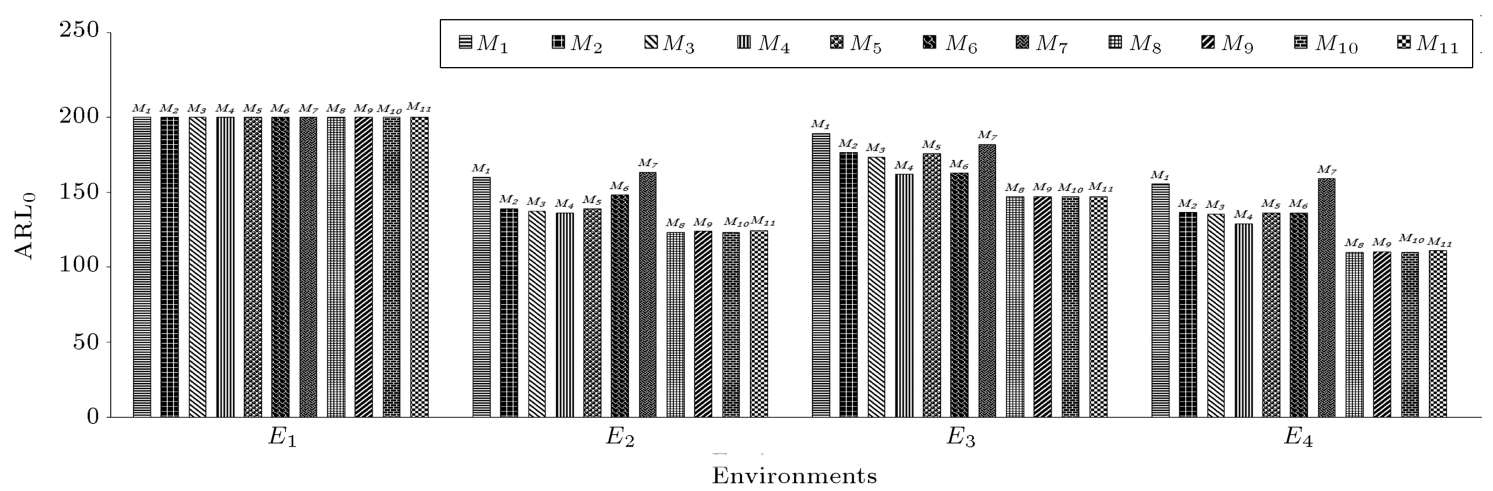

(a)

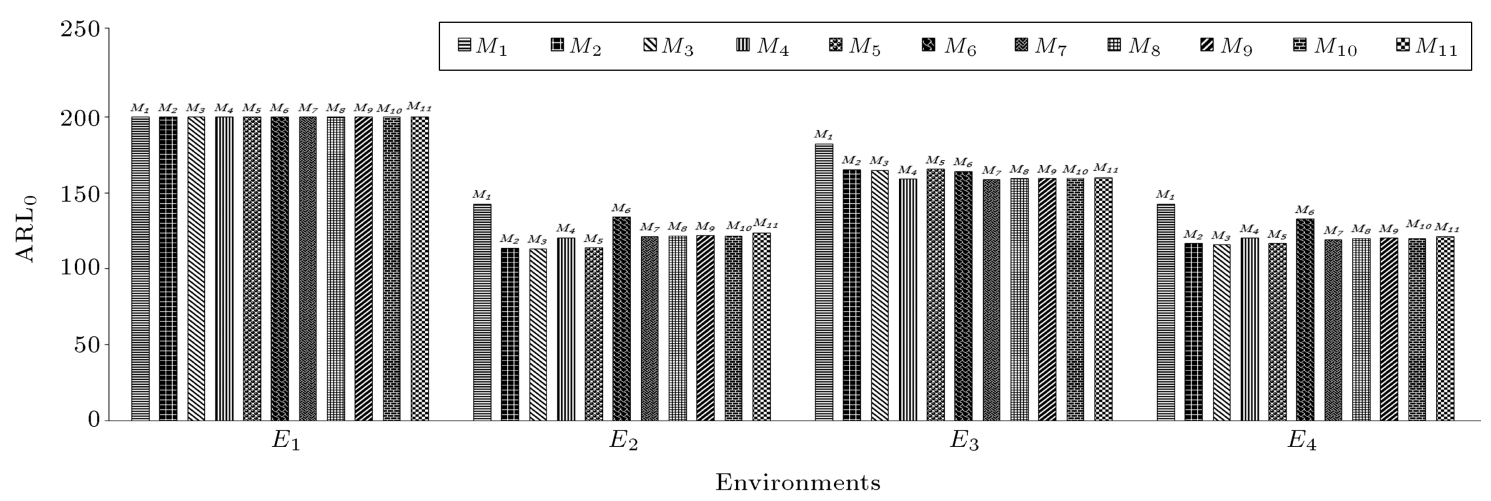

(b)

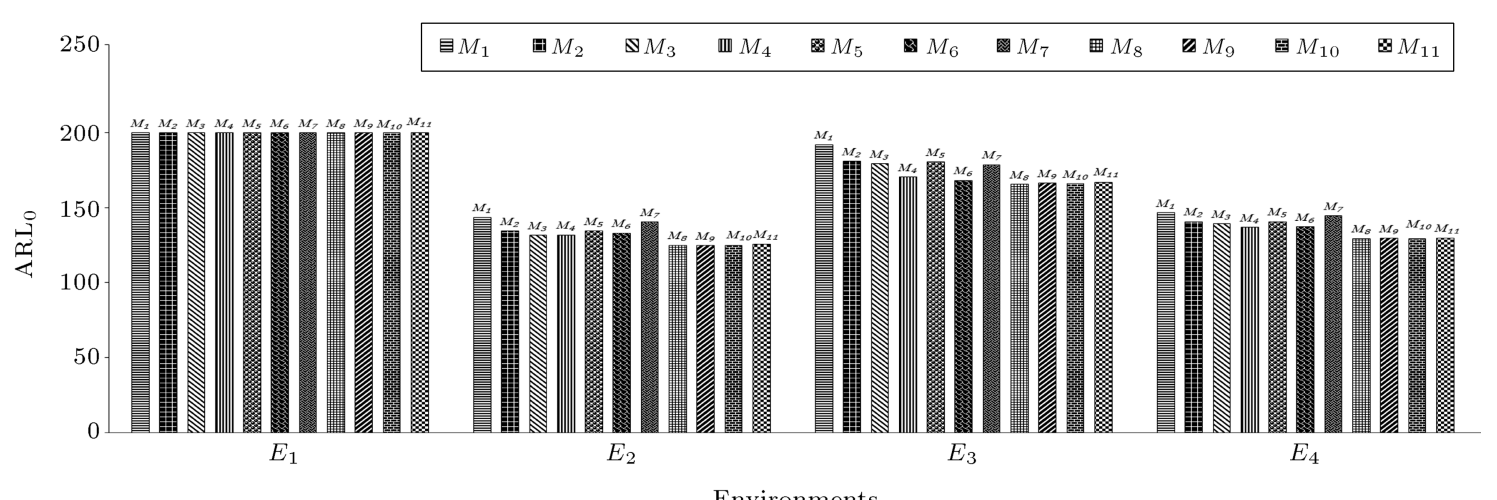

(c)

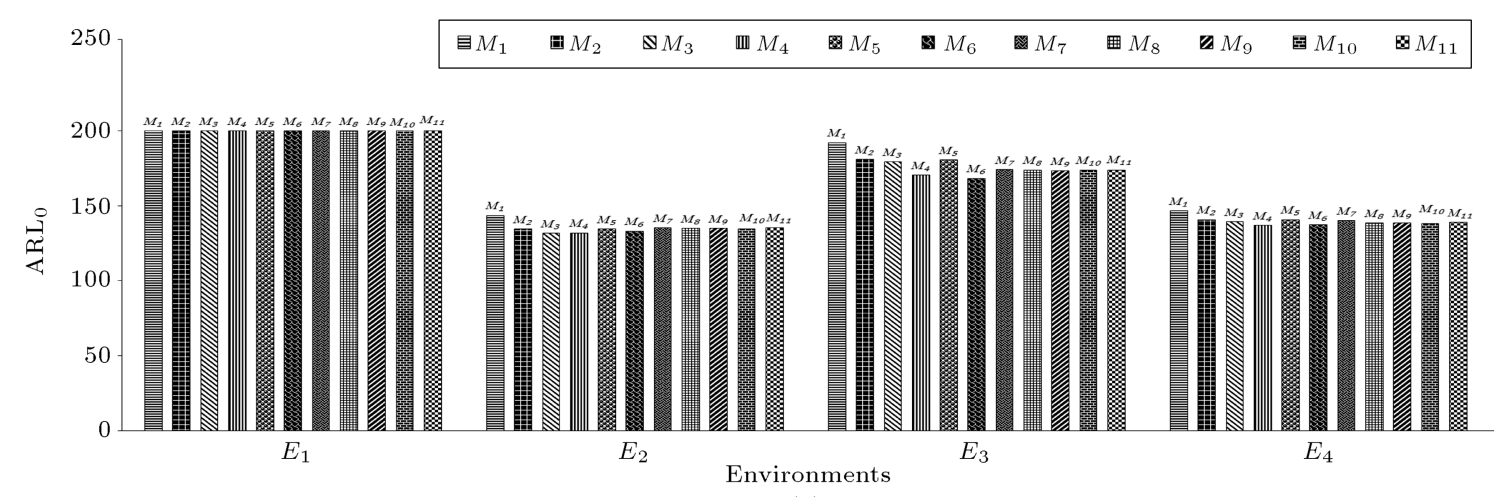

(d)

Figure 4. Median contaminated $\mathrm{ARL}_{0} \mathrm{~S}=200$, and $\lambda=0.50$ : (a) $\rho_{x y}=0.60, \rho_{z x}=0.30, \rho_{y z}=0.75$, and $n=5$; (b) $\rho_{x y}=0.75, \rho_{y z}=0.10, \rho_{z x}=0.10$, and $n=10 ;$ (c) $\rho_{x y}=0.50, \rho_{y z}=0.50, \rho_{z x}=0.10$, and $n=15$; and $(\mathrm{d}) \rho_{x y}=0.50$, $\rho_{y z}=0.10, \rho_{z x}=0.10$, and $n=15$. 


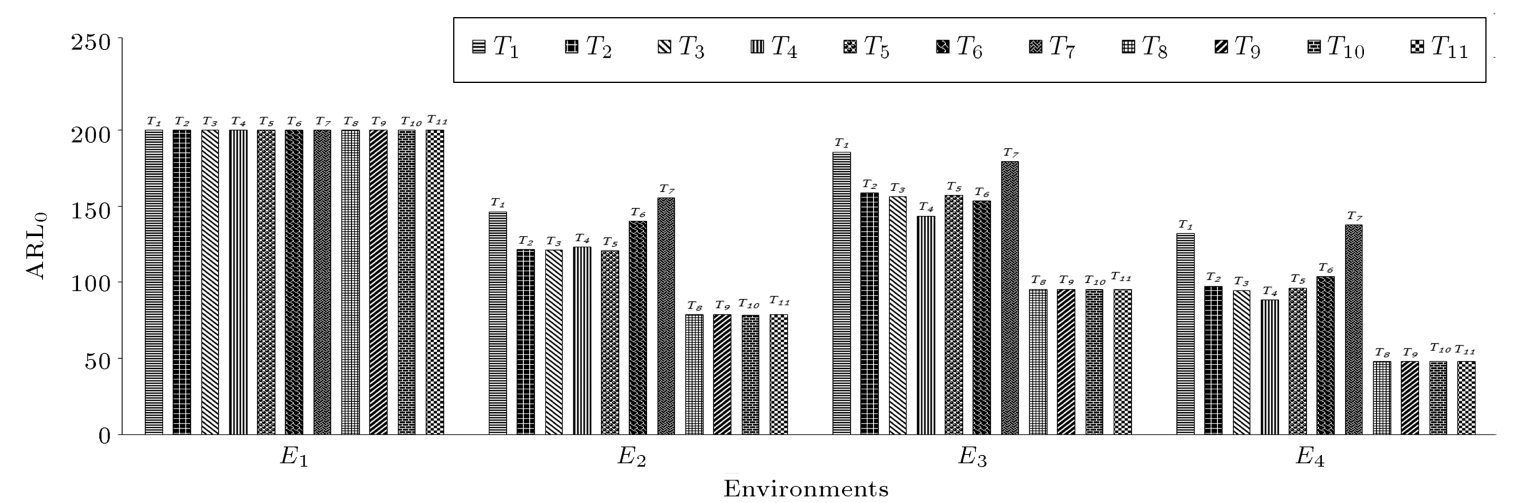

(a)

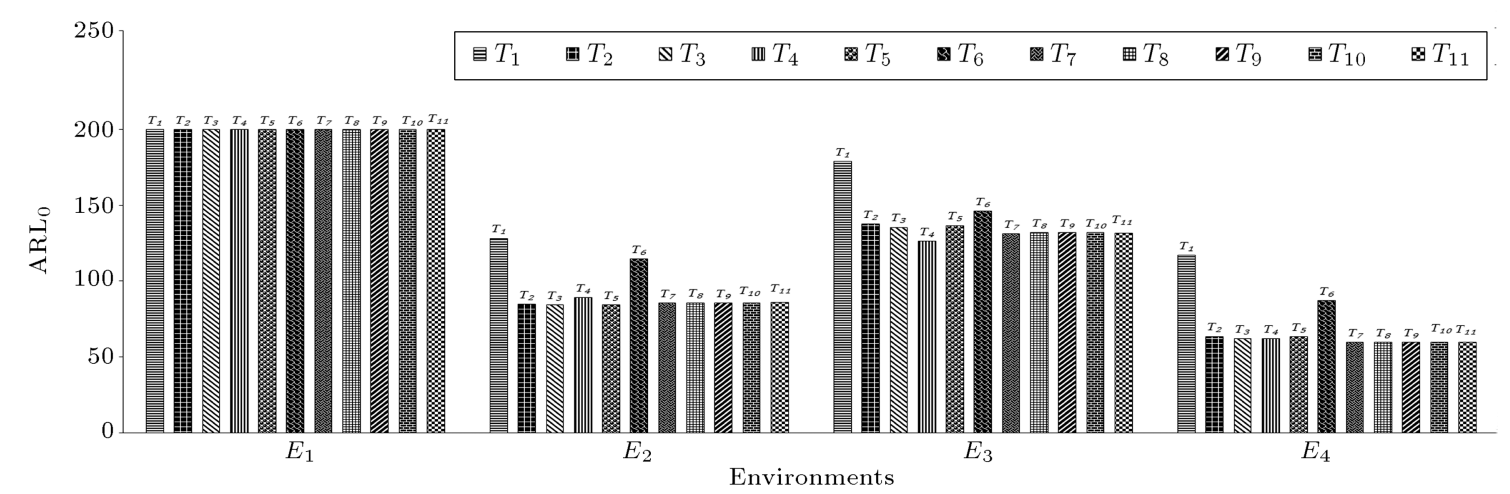

(b)

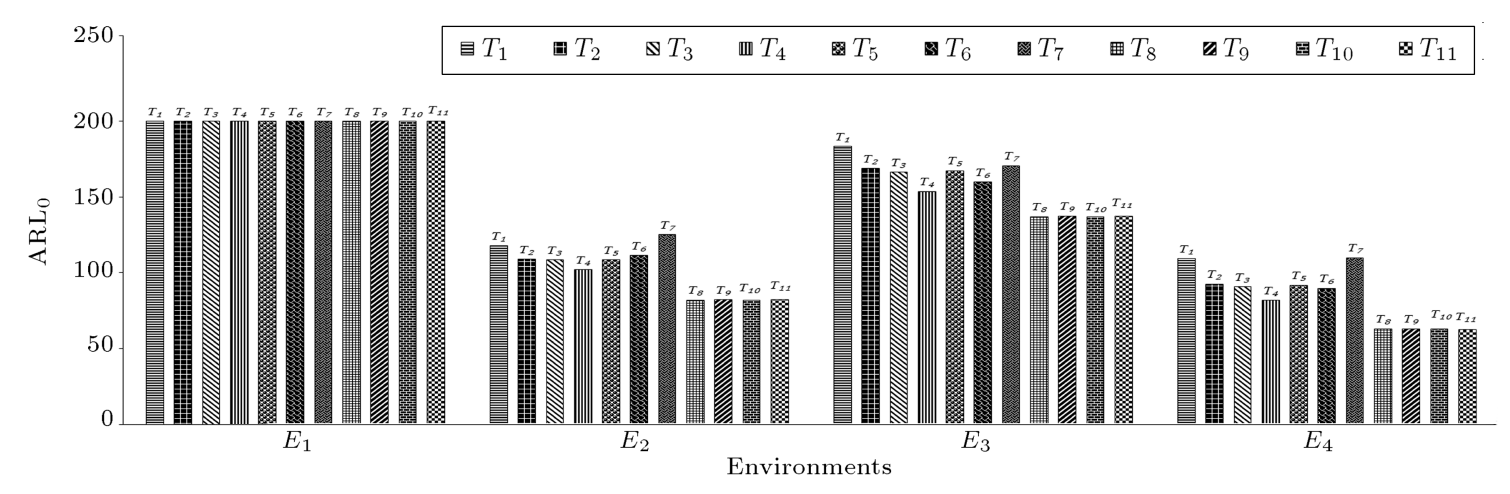

(c)

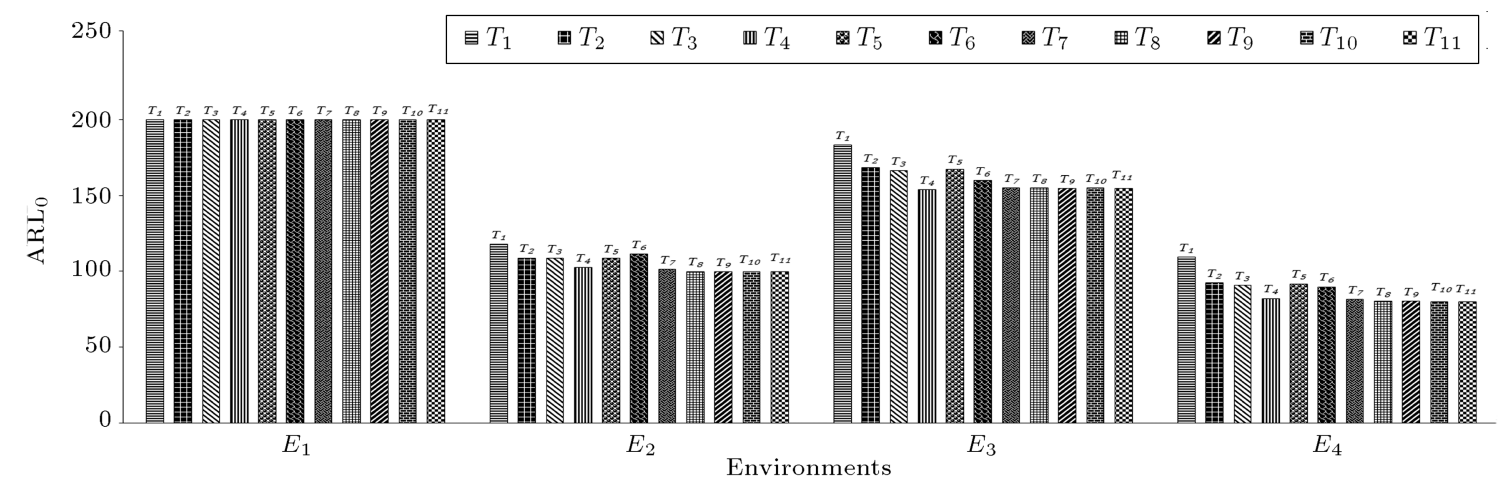

(d)

Figure 5. Mean contaminated $\mathrm{ARL}_{0} \mathrm{~s}=200$, and $\lambda=0.50$ : (a) $\rho_{x y}=0.60, \rho_{y z}=0.75, \rho_{z x}=0.30$, and $n=5$; (b) $\rho_{x y}=0.75, \rho_{y z}=0.10, \rho_{z x}=0.10$, and $n=10$; (c) $\rho_{x y}=0.50, \rho_{y z}=0.50, \rho_{z x}=0.10$, and $n=15$; and (d) $\rho_{x y}=0.50$, $\rho_{y z}=0.10, \rho_{z x}=0.10$, and $n=15$. 


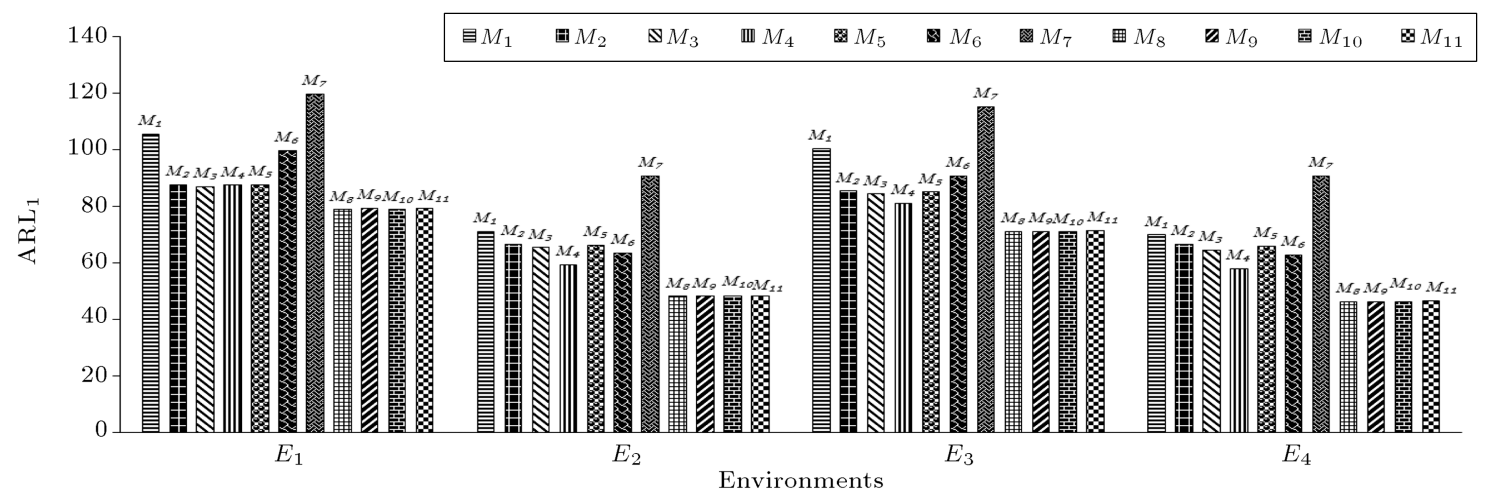

(a)

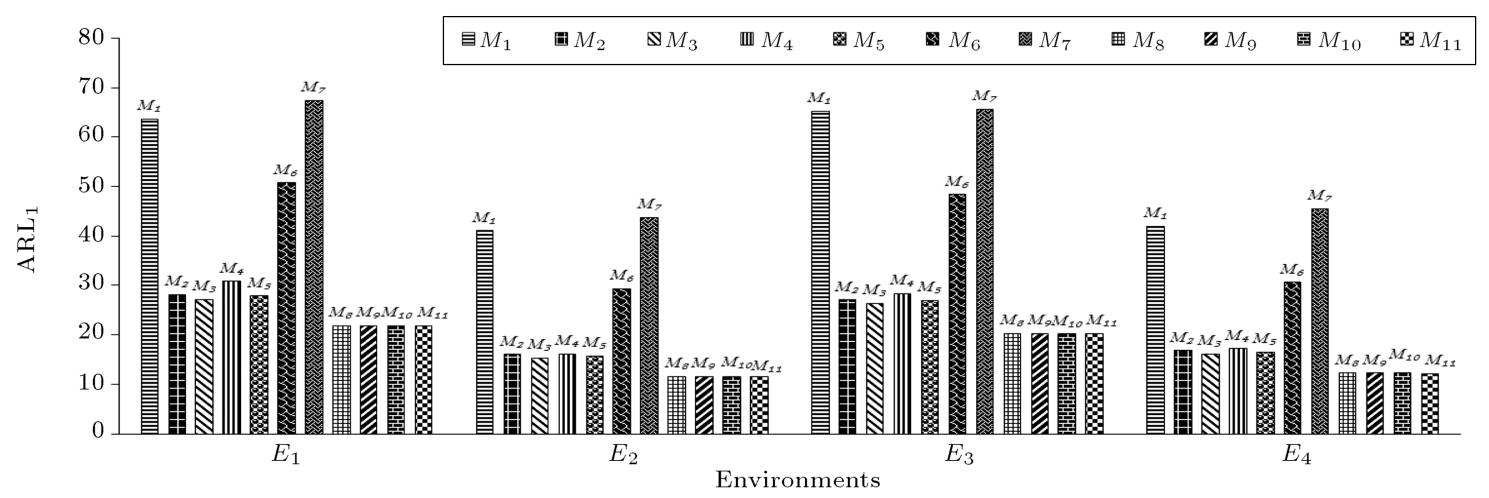

(b)

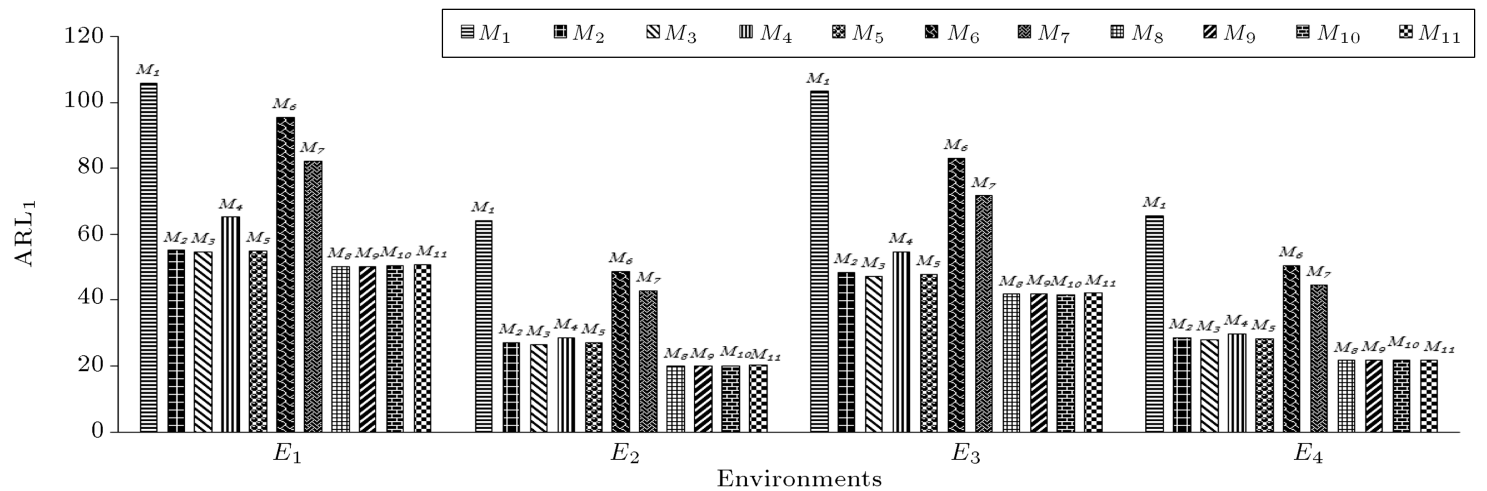

(c)

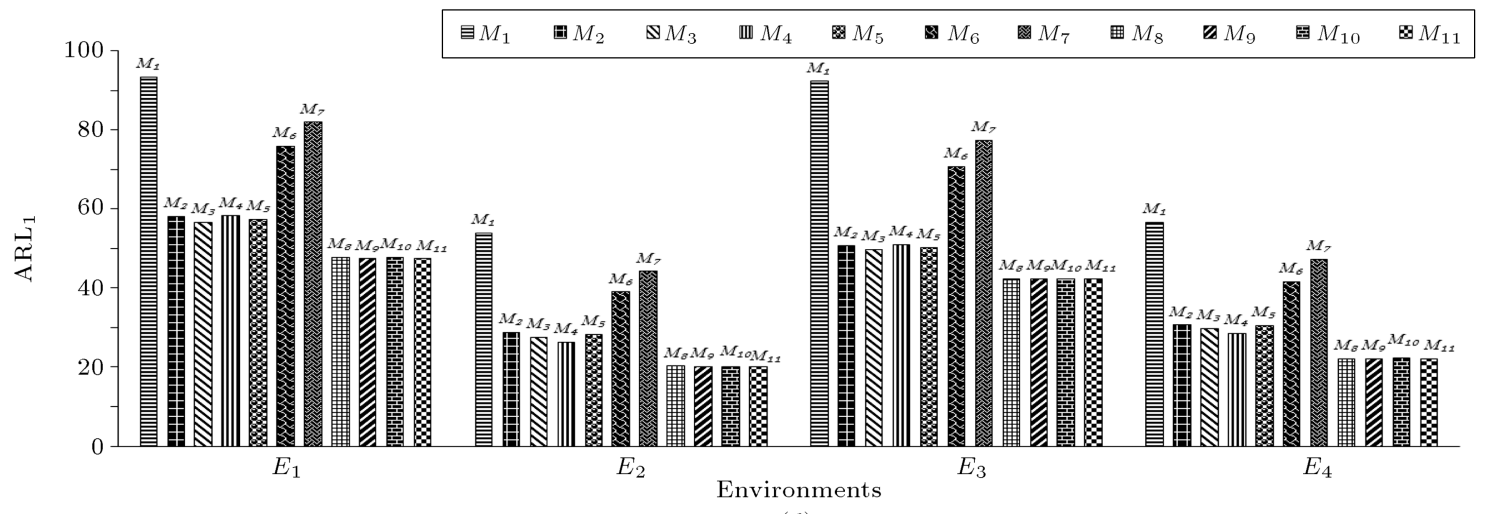

(d)

Figure 6. Median contaminated $\mathrm{ARL}_{1} \mathrm{~s}$ for varying scenarios at $\mathrm{ARL}_{0} \mathrm{~s}=200$, and $\lambda=0.50$ : (a) $\rho_{x y}=0.60, \rho_{y z}=0.75$, $\rho_{z x}=0.30, \delta=0.15$, and $n=5$; (b) $\rho_{x y}=0.90, \rho_{y z}=0.90, \rho_{z x}=0.90, \delta=0.15$, and $n=10 ;$ (c) $\rho_{x y}=0.90, \rho_{y z}=0.60$, $\rho_{z x}=0.30, \delta=0.10$, and $n=10 ;$ and $(\mathrm{d}) \rho_{x y}=0.90, \rho_{y z}=0.75, \rho_{z x}=0.50, \delta=0.10$, and $n=15$. 


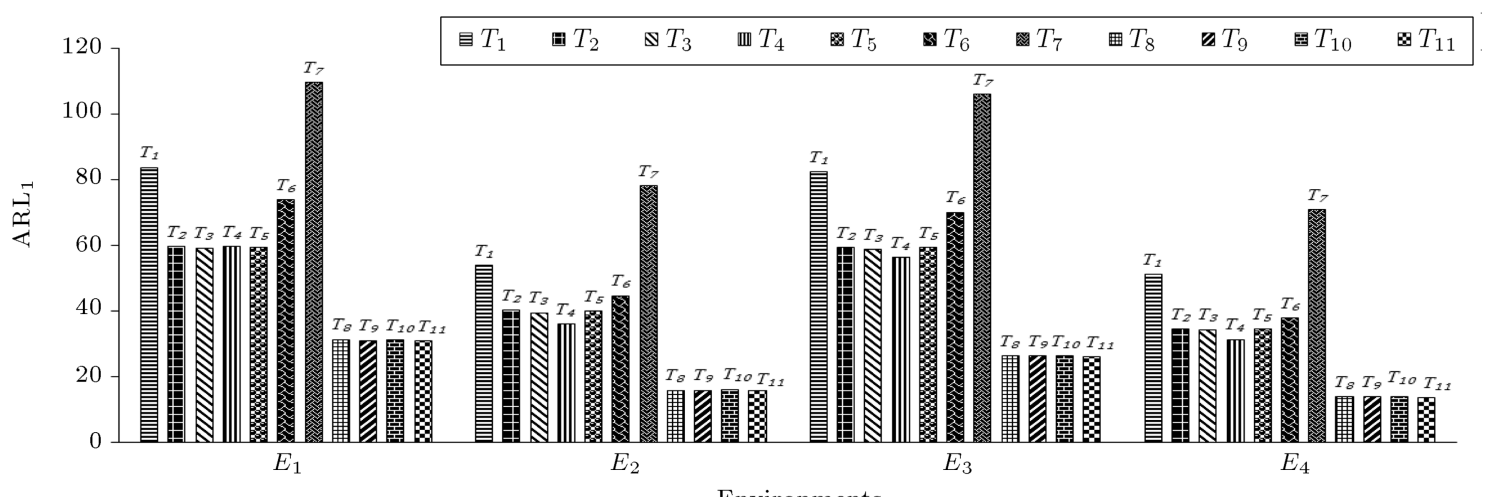

Environments

(a)

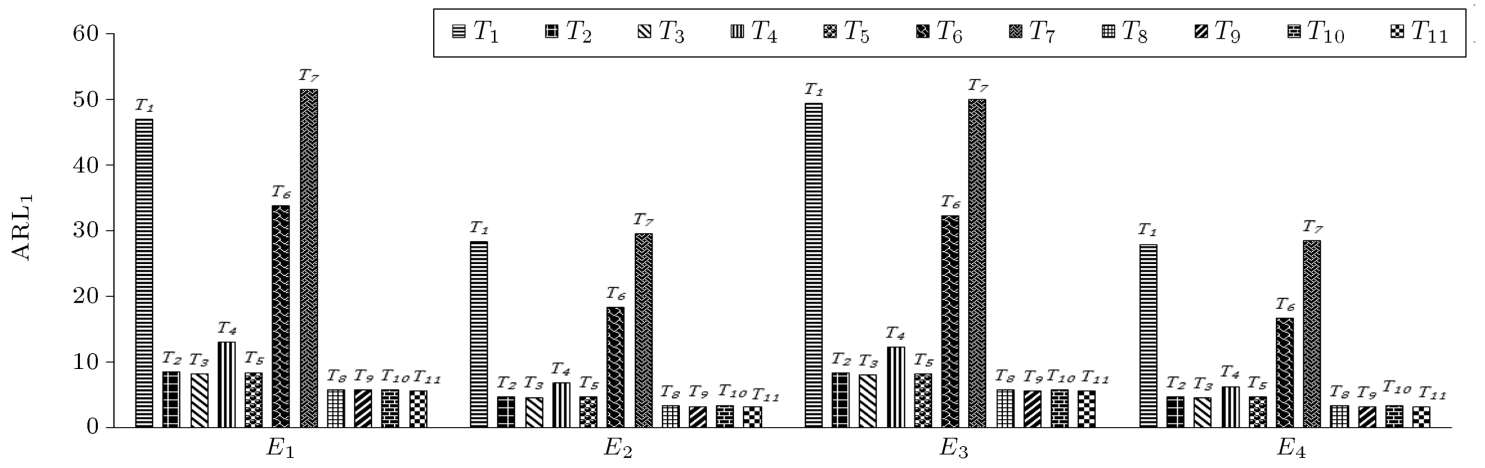

Environments

(b)

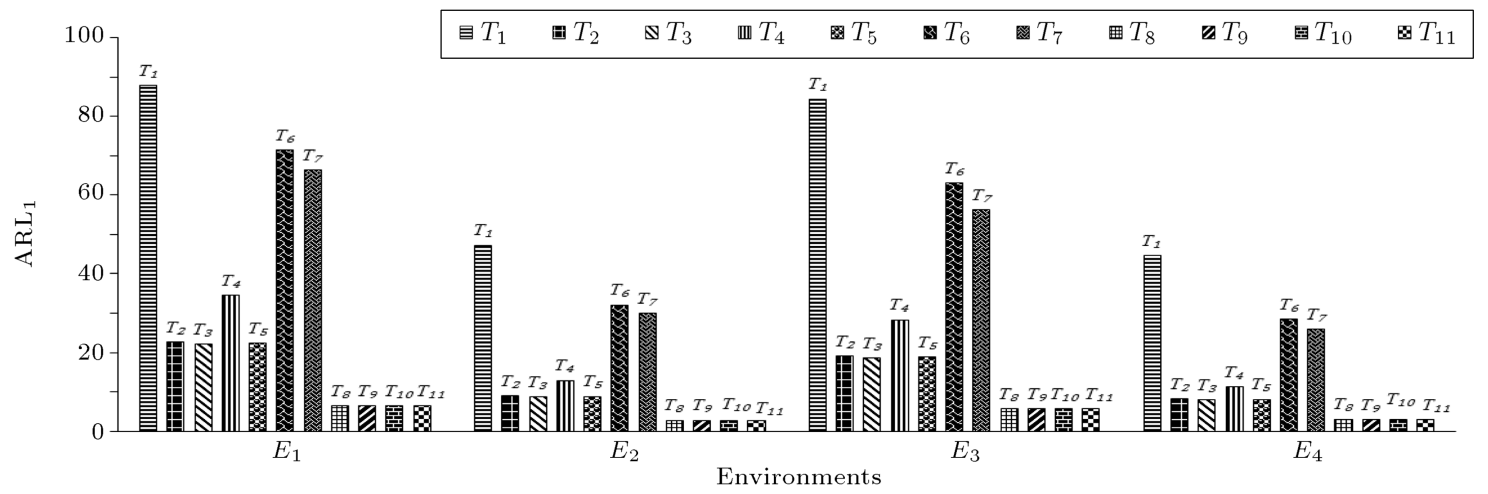

(c)

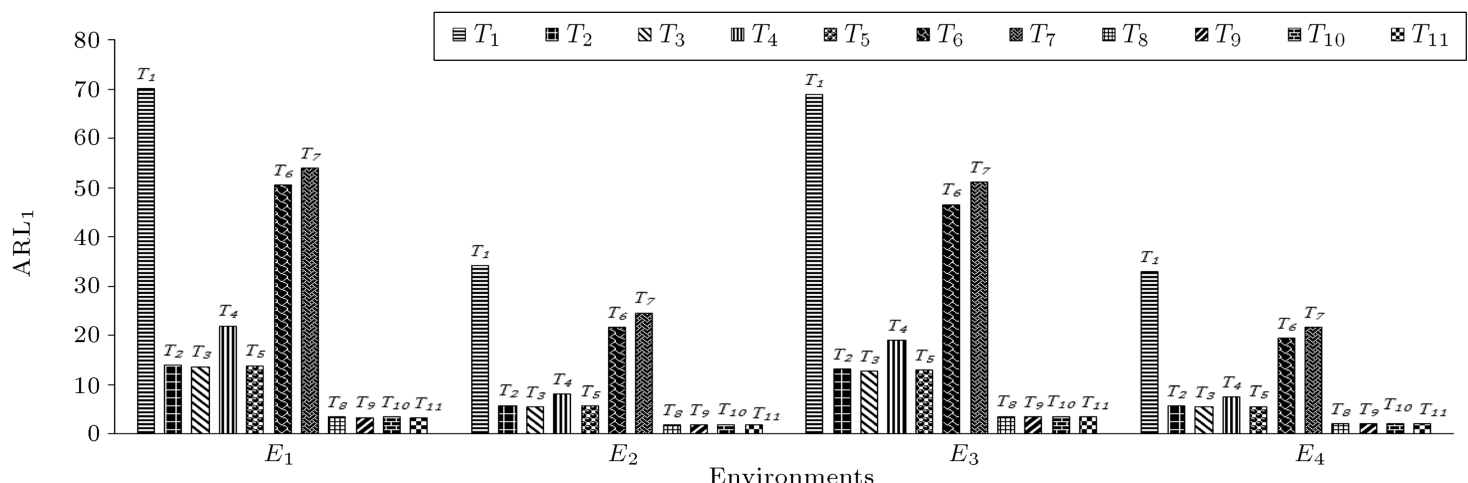

(d)

Figure 7. Mean contaminated $\mathrm{ARL}_{1} \mathrm{~s}$ for varying scenarios at $\mathrm{ARL}_{0} \mathrm{~s}=200$, and $\lambda=0.50:$ (a) $\rho_{x y}=0.60, \rho_{y z}=0.75$, $\rho_{z x}=0.30, \delta=0.15$, and $n=5$; (b) $\rho_{x y}=0.90, \rho_{y z}=0.90, \rho_{z x}=0.90, \delta=0.15$, and $n=10$; (c) $\rho_{x y}=0.90, \rho_{y z}=0.60$, $\rho_{z x}=0.30, \delta=0.10$, and $n=10$; and $(\mathrm{d}) \rho_{x y}=0.90, \rho_{y z}=0.75, \rho_{z x}=0.50, \delta=0.10$, and $n=15$. 
chamber as $X$, and pressure of gas as $Z$. different types of such practical situations may be seen in cf. [37].

\subsection{Simulated illustration}

For this example, we selected three charts: The first estimator, $M_{6}$, is selected based on one-auxiliary variable $X$, and the second estimator $M_{11}$ is chosen based on two auxiliary variables $X$ and $Z$. We compare the performance of these two selected estimators with that of the usual median estimator, $M_{1}$.

The trivariate normal distribution is used to generate two different data sets with mean vector, $\mu$, and variance-covariance matrix, $\Sigma$. Each data set contains 50 subgroups with sample sizes $n=5$ and 10 at two different levels of smoothing parameter $\lambda=0.75$ and $\lambda=0.25$. The mean vector, $\mu$, and variancecovariance matrix, $\Sigma$, can be shown as:

$$
\mu=\left(\begin{array}{c}
\mu_{y}+\delta \sigma_{y} \\
\mu_{x} \\
\mu_{z}
\end{array}\right)=\left(\begin{array}{c}
5+\delta \\
5 \\
5
\end{array}\right)
$$

and:

$$
\Sigma=\left(\begin{array}{ccc}
\sigma_{y}^{2} & \sigma_{x y} & \sigma_{y z} \\
\sigma_{x y} & \sigma_{x}^{2} & \sigma_{x z} \\
\sigma_{y z} & \sigma_{x z} & \sigma_{z}^{2}
\end{array}\right)=\left(\begin{array}{ccc}
1 & 0.90 & 0.90 \\
0.90 & 1 & 0.90 \\
0.90 & 0.90 & 1
\end{array}\right)
$$

In these sample data sets, the first 30 observations are generated from the in-control scenario, that is, $\delta=0$, while the last 20 observations are generated from an out-of-control scenario with shifts $\delta=1.0$ for $\lambda=0.75$ and $\delta=0.75$ for $\lambda=0.25$. The computed values of $M_{1}$, $M_{6}$, and $M_{11}$ based on these data sets are presented in Tables A.1 and A.2 (see Appendix).

Figure 8 displays a graphical representation of control charting structures of $M_{1}, M_{6}$, and $M_{11}$ based on different $\lambda$ values and sample sizes. In this figure, $\mathrm{UCL}_{i}$ and $\mathrm{LCL}_{i}$ represent the upper and lower control limits for control charts based on estimators $M_{i}(i=$ $1,6,11)$. It is observed from Figure $8(\mathrm{a})$ that the control structure based on the estimator $M_{1}$ detects 4 out-of-control signals at points 40,45, 49, and 50, while control structure based on estimator $M_{6}$ detects 14 out-of-control signals at points $32,33,35-39,42,44$, 45, and 47-50 and control structure based on estimator $M_{11}$ detects 17 out-of-control signals at points 31,32 , 34 , and $37-50$ with $\lambda=0.75, \delta=1.0$, and $n=5$. This noticeably points out that the control chart based on estimator $M_{6}$ detects 10 more out of control points than the control chart based on estimator $M_{1}$ does and the control chart based on estimator $M_{11}$ detects 13 and 3 more out-of-control points than $M_{1}$-based control structure and $M_{6}$-based control structure do, respectively. Moreover, the control chart based on estimator $M_{11}$ signals earlier than two other control charts does, which supports our findings in this paper, i.e. the control charts based on two auxiliary variables are more efficient than the control charts which are based on one-auxiliary variable.

One can also notice that Figure $8(\mathrm{~b})$ gives more detections than Figure 8(a) does. This indicates that detection capability of charts increases with an increase in sample size; for example, when $n=5, M_{1}, M_{6}$, and $M_{11}$ detect 4,14 , and 17 out-of-control points, respectively, while 14,18 , and 20 out-of-control points are detected for $n=10$ (Figure $8(\mathrm{~b})$ ).

From the comparison of Figure 8(a) and (b) with Figure $8(\mathrm{c})$ and (d), it can be noted that control structures are more efficient with the lower value of $\lambda$. For the same sample size, $n=5$, charting structures based on $M_{1}, M_{6}$, and $M_{11}$, respectively, detect 4,14 , and 17 points when $\lambda=0.75$ and $\delta=1.0$ (Figure $8(\mathrm{a})$ ), while they detect 12,18 , and 19 points, respectively, when $\lambda=0.25$ and $\delta=0.75$ (Figure $8(\mathrm{c})$ ). A similar type of behavior at $n=10$ and higher detection ability of $M_{11}$ chart can be observed in these figures.

\subsection{Case study}

In order to demonstrate the application of the proposed auxiliary information based median control charts $\left(T_{i}\right)$, we provide an example here for four different combinations of charts. In each combination, we select one chart based on two auxiliary variables and one chart based on single auxiliary variable, and make a comparison with usual median control chart. For the mentioned purpose, we consider the data-set based on the non-iso-thermal continuous stirred tank chemical reactor model, namely, the CSTR process, originally proposed by Marlin [65], which has been widely used as a benchmark in fault detection and diagnosis (cf. [66,67]). The CSTR process comprises nine process variables; the details of variables may be found in [66,67]. For our example, we have considered $\mathrm{CA}$ (outlet concentration of the product measured in $\mathrm{kmole} / \mathrm{m}^{3}$ ) as $Y, T_{O}$ (inlet temperature measured in Kelvin) as $X$, and $F_{A}$ (flow rate of reactant $A$ measured in $\mathrm{m}^{3} / \mathrm{min}$ ) as $Z$. the mentioned data-set originally contains 1024 values that are collected at sampling intervals of half minute. The initial 512 values (the first half of the data) are from the in-control state with shift zero, whereas shift $(\delta=0.15)$ has been introduced in $\mu_{y}$ for the second half of data-set to monitor process location parameter. We have considered the mentioned trivariate data-set in the form of 102 sub-groups each of size $n=10$ (that is by making each group after 5 minutes). The control limits for all charts have been constructed for $\mathrm{ARL}_{0}=200$ with $(\lambda=0.75)$ based on in-control data. The values of three charting statistics are computed and the results are displayed in the form of control chart, Figure 9(a)-(d), by plotting charting statistic values on the vertical axis and sample numbers 


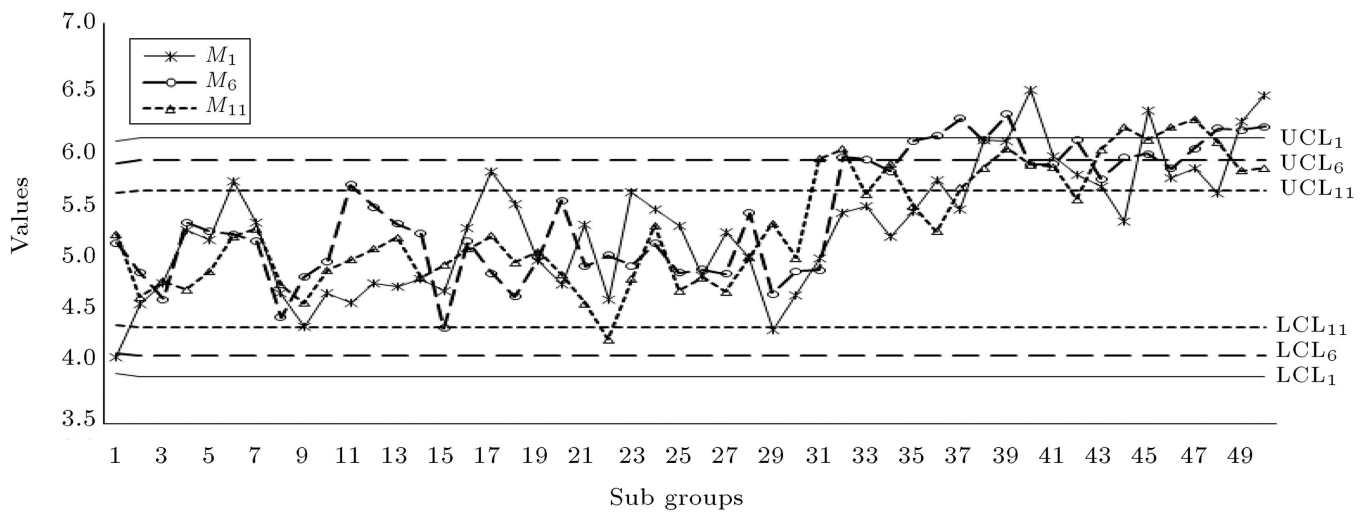

(a)

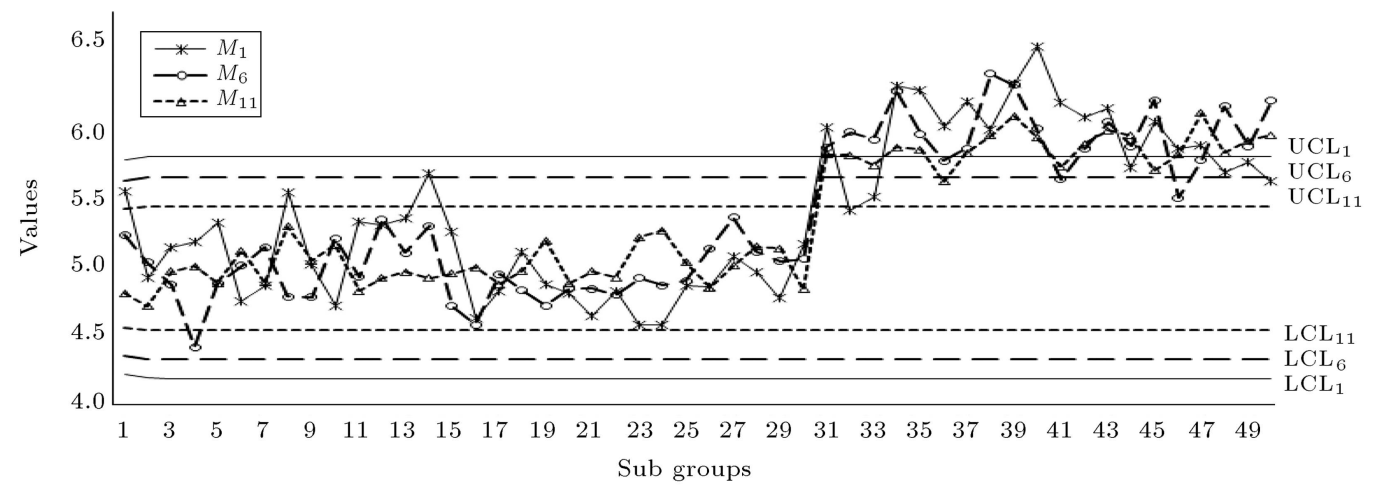

(b)

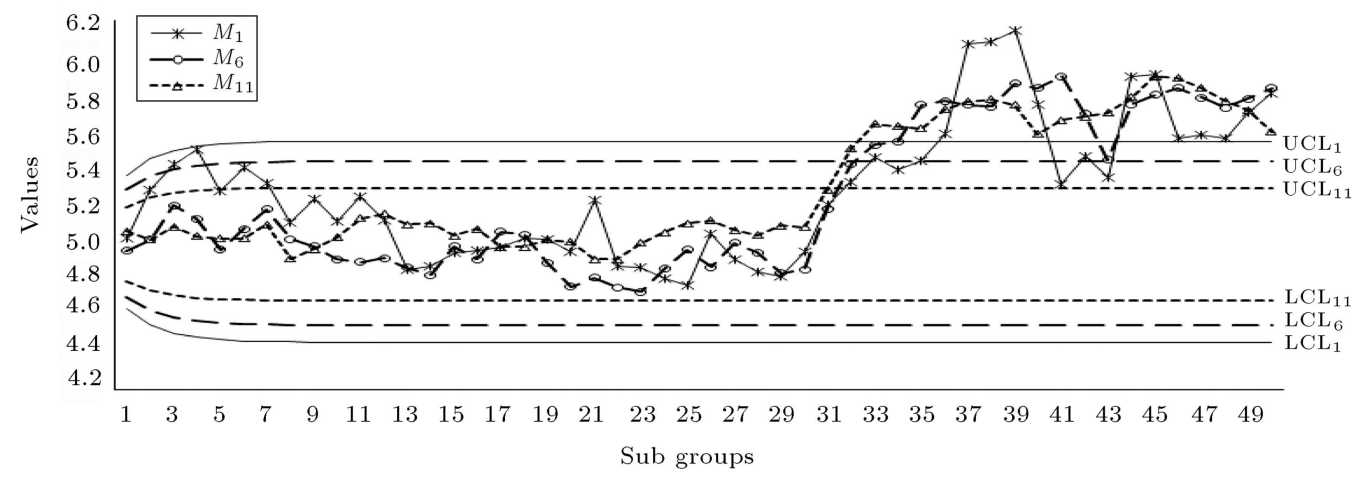

(c)

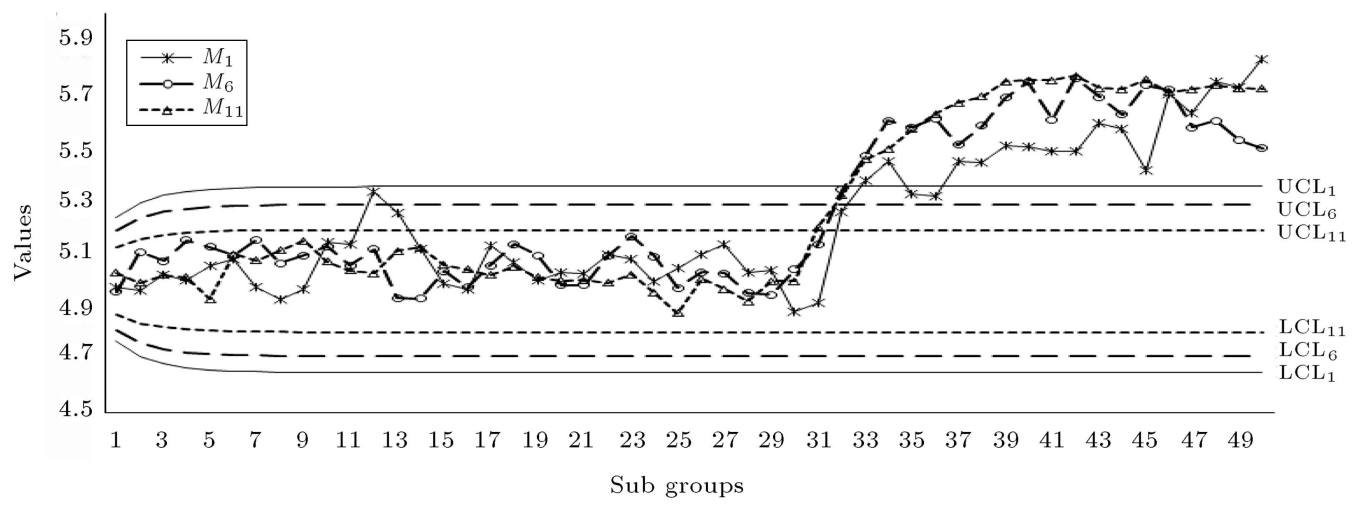

(d)

Figure 8. Control chart display for $M_{1}, M_{6}$ and $M_{11}$ : (a) $n=5, \lambda=0.75, \delta=1.0$ and $\rho_{x y}=0.90, \rho_{y z}=0.90, \rho_{z x}=0.90$; (b) $n=10, \lambda=0.75, \delta=1.0$ and $\rho_{x y}=0.90, \rho_{y z}=0.90, \rho_{z x}=0.90 ;$ (c) $n=5, \lambda=0.25, \delta=0.75$ and $\rho_{x y}=0.90$, $\rho_{y z}=0.90, \rho_{z x}=0.90 ;$ and (d) $n=10, \lambda=0.25, \delta=0.75$ and $\rho_{x y}=0.90, \rho_{y z}=0.90, \rho_{z x}=0.90$. 

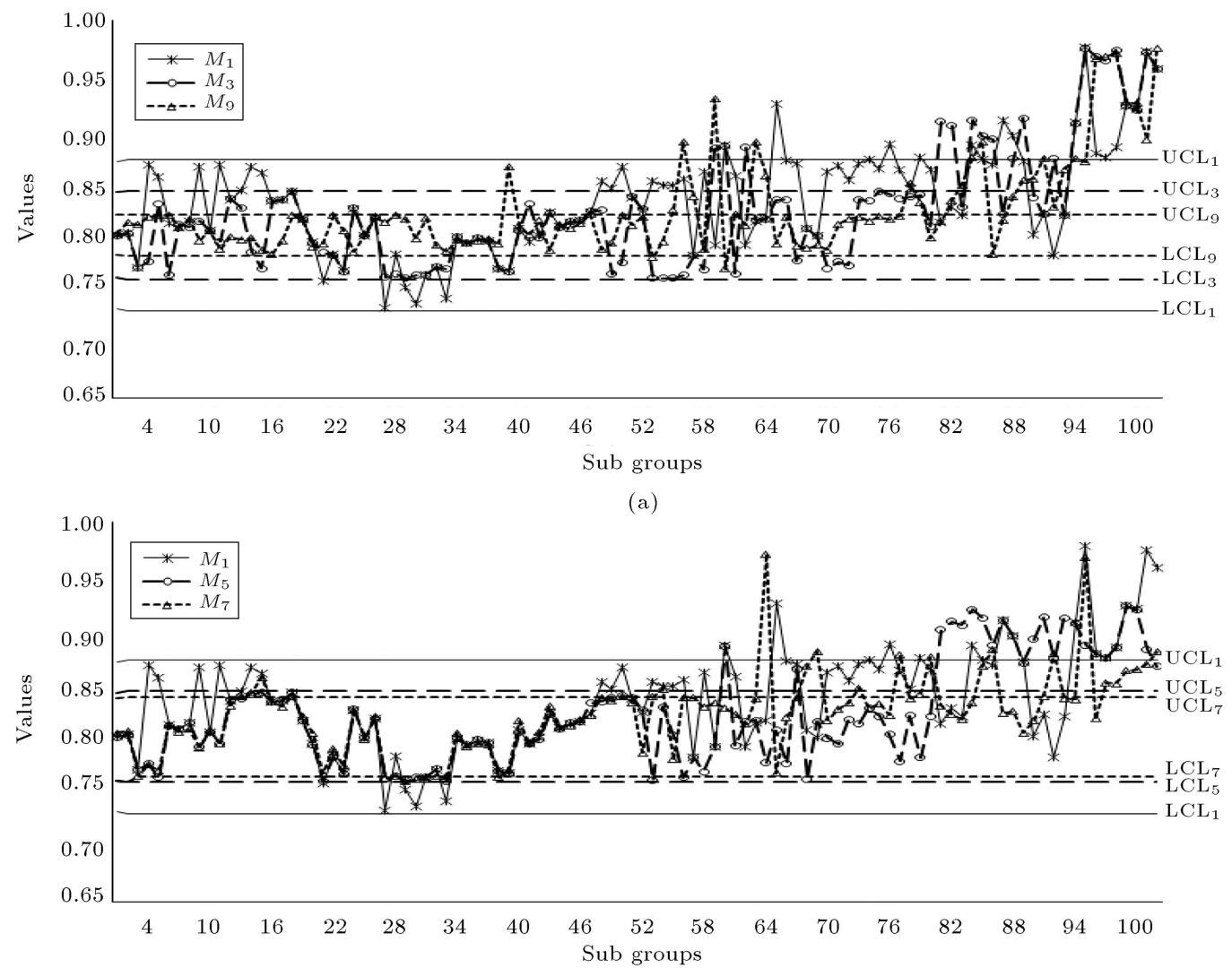

(b)
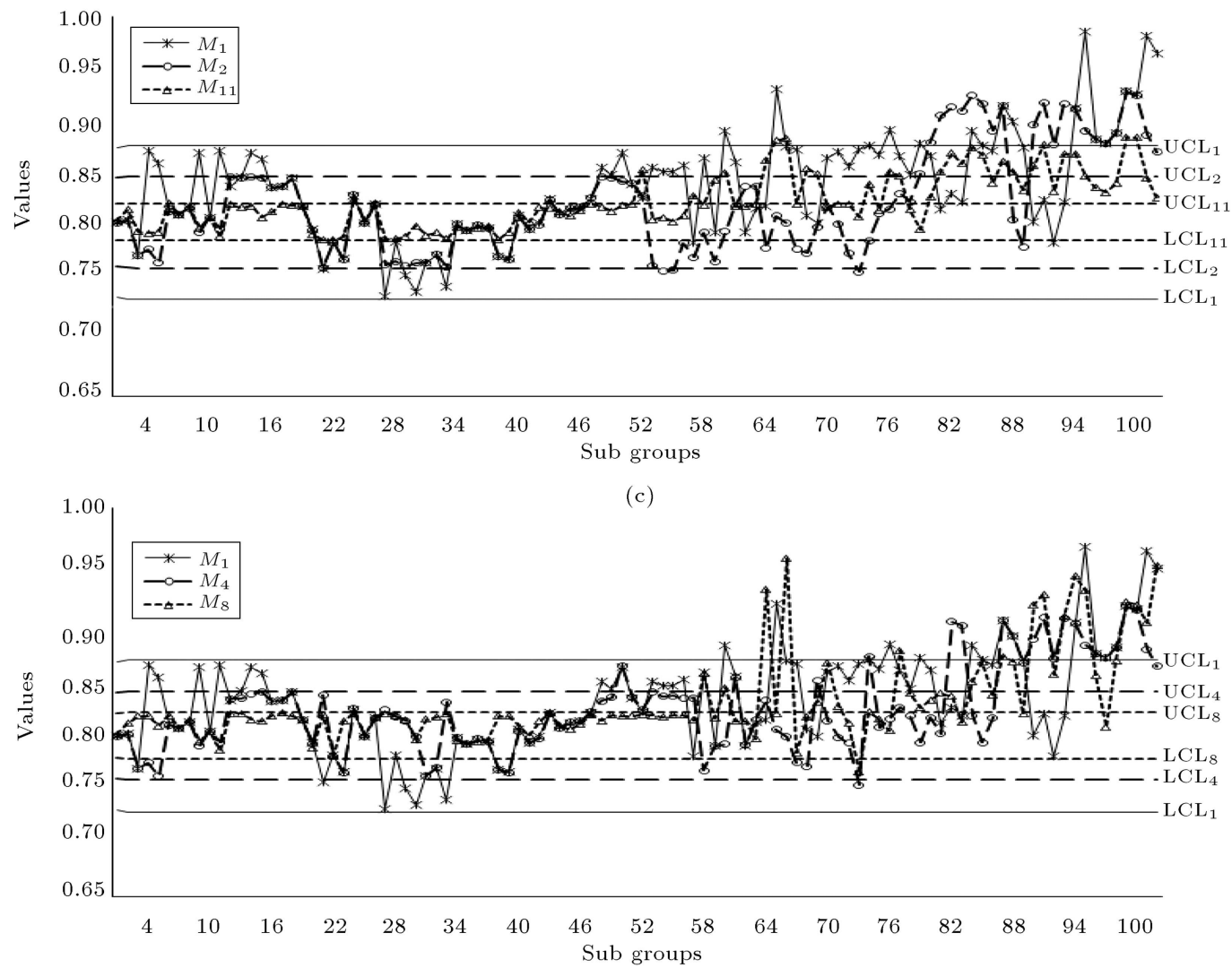

(d)

Figure 9. Control chart display with $n=10, \lambda=0.75$, and $\delta=0.15$ for (a) $M_{1}, M_{3}$ and $M_{9}$; (b) $M_{1}, M_{5}$ and $M_{7}$; (c) $M_{1}$, $M_{2}$ and $M_{11}$; and (d) $M_{1}, M_{4}$ and $M_{8}$. 
(sub-groups) on the horizontal axis. After the 51st subgroup, the details of detection ability of each figure can be observed as:

- In Figure $9(\mathrm{a})$, charts $T_{1}, T_{3}$, and $T_{9}$ have detected 16,20 , and 31 out-of-control signals, respectively;

- In Figure $9(\mathrm{~b})$, charts $T_{1}, T_{5}$, and $T_{7}$ have detected 16, 24, and 28 out-of-control signals, respectively;

- In Figure $9(\mathrm{c})$, charts $T_{1}, T_{2}$, and $T_{11}$ have detected 16,25 , and 36 out-of-control signals, respectively;

- In Figure $9(\mathrm{~d})$, charts $T_{1}, T_{4}$, and $T_{8}$ have detected 16,23 , and 32 out-of-control signals, respectively.

It shows that the control charts based on two auxiliary variables have the best detection ability followed by one-auxiliary information based control charts and usual median control chart, respectively, which is in accordance with the findings in Section 4.

\section{Conclusions and future recommendations}

Variations are an integral part of every process and these variations need our attention to improve the quality of the process. We use control charts in order to classify these variations as natural and unnatural. For location, monitoring mean is the most commonly used measure. However, median serves as a better alternative when processes face sudden outliers. Moreover, information about auxiliary variables helps to enhance the precision of the estimators and, hence, the charting structure. In this article, we proposed the median-based EWMA control charts to monitor the location parameter. With the help of one- and twoauxiliary variables, we designed the mean and median based EWMA control charts and compared their performances under contaminated and un-contaminated process environments. Based on these estimators, we investigated the EWMA control charting structures and carried out detailed run length using different performance measures ARL, EQL, RARL, and PCI. Based on our evaluation, in the uncontaminated environment, mean control charts are better than median control charts. The detection ability of mean control charts is better than that of median control charts. However, under the contaminated environments, median control charts are better than mean control charts. The resistance ability of median control charts is higher than that of mean control charts in the presence of outliers. By comparison of all median estimators, results also displayed the overall dominance of control charting structures based on two-auxiliary variables. As the analysis of EWMA control charts also includes $\lambda=1$ among the different values of $\lambda$ (which is a special case of Shewhart control charts), it is worth mentioning that, for the same estimators, results of
EWMA control charts are better than those of Shewhart control charts. These major findings can also be observed from the illustrative example. From this example, we can also observe that if the size increases, these charts will be more efficient in detecting shifts in the process. Moreover, the scope of this study can be further extended to other types of control charts, such as multivariate EWMA and CUSUM control charts. Different run rules can also be used to enhance the detection abilities of these charting structures.

\section{Acknowledgement}

The first author is thankful to National Natural Science Foundation of China (11371077 and 11471065) for their financial support. This work was also supported by the Fundamental Research Funds for the Central Universities in China (DUT15LK28). The author Muhammad Riaz is indebted to King Fahd University of Petroleum and Minerals (KFUPM), Dhahran, Saudi Arabia, for providing excellent research facilities. The authors are thankful to the anonymous reviewers and Editor for the constructive comments that helped in improving the last version of the paper.

\section{Nomenclature}

\begin{tabular}{ll} 
ARL & Average Run Length \\
ARL $_{0}$ & In-control ARL \\
ARL $_{1}$ & Out-of-control ARL \\
CSTR & Continuous Stirred Tank Reactor \\
CL & Central Line \\
$C_{x}$ & Coefficient of variations for $X$ \\
$C_{z}$ & Coefficient of variations for $Z$ \\
EQL & Extra Quadratic Loss \\
$f_{x}(x)$ & Marginal density function of $X$ \\
$f_{y}(y)$ & Marginal density function of $Y$ \\
$f_{z}(z)$ & Marginal density function of $Z$ \\
$M_{y}$ & Median of $Y$ \\
$M_{x}$ & Median of $X$ \\
$M_{z}$ & Median of $Z$ \\
$\hat{M}_{y}$ & Sample median of $Y$ \\
$\hat{M}_{x}$ & Sample median of $X$ \\
$\hat{M}_{z}$ & Sample median of $Z$ \\
$n$ & Sample size \\
$P_{11}(x, y)$ & Pr $\left(X \leq M_{x}\right.$ and $\left.Y \leq M_{y}\right)$ \\
$P_{11}(y, z)$ & Pr $Y \leq M_{y}$ and $\left.Z \leq M_{z}\right)$ \\
$P_{11}(z, x)$ & Pr $Z \leq M_{z}$ and $\left.X \leq M_{x}\right)$ \\
$\mathrm{LCL}_{\mathrm{UCL}}$ & Lower Control Limit \\
& Upper Control Limit \\
\hline &
\end{tabular}




\section{References}

1. Does, R.J., Roes, K.C. and Trip, A., Statistical Process Control in Industry, Kluwer Academic Publishers: Dordrecht, The Netherlands (1999).

2. Montgomery, D.C., Introduction to Statistical Quality Control, John Wiley \& Sons (2007).

3. Page, E. "Continuous inspection schemes", Biometrika, pp. 100-115 (1954).

4. Roberts, S. "Control chart tests based on geometric moving averages", Technometrics, 1(3), pp. 239-250 (1959).

5. Abbas, N., Riaz, M. and Does, R.J. "Enhancing the performance of EWMA charts", Quality and Reliability Engineering International, 27(6), pp. 821-833 (2011).

6. Haq, A., Brown, J., Moltchanova, E., and Al-Omari, A.I. "Improved exponentially weighted moving average control charts for monitoring process mean and dispersion", Quality and Reliability Engineering International, 31(2), pp. 217-237 (2015).

7. Capizzi, G. and Masarotto, G. "An adaptive exponentially weighted moving average control chart", Technometrics, 45(3), pp. 199-207 (2003).
8. Abbas, N., Riaz, M., and Does, R.J. "Mixed exponentially weighted moving average-cumulative sum charts for process monitoring", Quality and Reliability Engineering International, 29(3), pp. 345-356 (2013).

9. Haq, A. "A new hybrid exponentially weighted moving average control chart for monitoring process mean", Quality and Reliability Engineering International, 29(7), pp. 1015-1025 (2013).

10. Stemann, D. and Weihs, C. "The EWMA-X-S-control chart and its performance in the case of precise and imprecise data", Statistical Papers, 42(2), pp. 207-223 (2001).

11. Haq, A., Brown, J., and Moltchanova, E. "Improved fast initial response features for exponentially weighted moving average and cumulative sum control charts", Quality and Reliability Engineering International, 30(5), pp. 697-710 (2014).

12. Abbas, N., Riaz, M., and Does, R.J. "Memorytype control charts for monitoring the process dispersion", Quality and Reliability Engineering International, 30(5), pp. 623-632 (2014a).

13. Abbasi, S.A., Riaz, M., Miller, A., Ahmad, S., and Nazir, H. Z. "EWMA dispersion control charts for normal and non-normal processes", Quality and Reliability Engineering International, 31(8), pp. 1691-1704 (2015).

14. Abbasi, S.A. and Miller, A. "MDEWMA chart: an efficient and robust alternative to monitor process dispersion", Journal of Statistical Computation and Simulation, 83(2), pp. 247-268 (2013).

15. Machado, M.A. and Costa, A.F. "The double sampling and the EWMA charts based on the sample variances", International Journal of Production Economics, 114(1), pp. 134-148 (2008).

16. Castagliola, P., Celano, G., Fichera, S., and Giuffrida, F. "A variable sampling interval S2-EWMA control chart for monitoring the process variance", International Journal of Technology Management, 37(1-2), pp. 125-146 (2006).

17. Stoumbos, Z.G.B. and Reynolds Jr, M.R. "Robustness to non-normality and autocorrelation of individuals control charts", Journal of Statistical Computation and Simulation, 66(2), pp. 145-187 (2000).

18. Chen, G., Cheng, S.W., and Xie, H. "Monitoring process mean and variability with one EWMA chart", Journal of Quality Technology, 33(2), pp. 223-233 (2001).

19. Shu, L. and Jiang, W. "A new EWMA chart for monitoring process dispersion", Journal of Quality Technology, 40(3), pp. 319-331 (2008).

20. Riaz, M. and Does, R.J. "A process variability control chart", Computational Statistics, 24(2), pp. 345-368 (2009). 
21. Niaki, S.T.A., Malaki, M., and Ershadi, M.J. "A particle swarm optimization approach on economic and economic-statistical designs of MEWMA control charts", Scientia Iranica, 18(6), pp. 1529-1536 10.1016/j.scient.2011.09.007 (2011).

22. Amiri, F., Noghondarian, K., and Noorossana, R. "Economic-statistical design of adaptive X-bar control chart: a Taguchi loss function approach", Scientia Iranica, 21(3), pp. 1096-1104 (2014).

23. Abujiya, M., Lee, M., and Riaz, M. "New EWMA S2 control charts for monitoring process dispersion", Scientia Iranica, 24(1), pp. 378-389 (2017). DOI: $10.24200 /$ sci.2017.4041.

24. Kanji, G. and Arif, O.H. "Median rankit control chart by the quantile approach", Journal of Applied Statistics, 27(6), pp. 757-770 (2000).

25. Kanji, G. and Arif, O.H. "Median rankit control chart for Weibull distribution", Total Quality Management, 12(5), pp. 629-642 (2001).

26. Chen, Y.-K. and Chiou, K.-C. "An evaluation of median Rankit control charts", Systems, Man and Cybernetics, 2008. SMC 2008. IEEE International Conference on, IEEE, pp. 3601-3605 (2008).

27. Yang, L., Pai, S., and Wang, Y.-R. "A novel CUSUM median control chart", Proceedings of International Multiconference of Engineers and Computer Scientists, Citeseer (2010).

28. Sheu, S.-H. and Yang, L. "The generally weighted moving average control chart for monitoring the process median", Quality Engineering, 18(3), pp. 333-344 (2006).

29. Sheu, S.-H. and Yang, L. "The generally weighted moving average median control chart", Quality Technology and Quantitative Management, 3(4), pp. 455471 (2006).

30. Castagliola, P. "An-EWMA control chart for monitoring the process sample median", International Journal of Reliability, Quality and Safety Engineering, 8(02), pp. 123-135 (2001).

31. Castagliola, P., Maravelakis, P.E., and Figueiredo, F.O. "The EWMA median chart with estimated parameters", IIE Transactions, 48(1), pp. 66-74 (2016).

32. Singh, R. and Mangat, N.S., Elements of Survey Sampling, 15, Springer Science \& Business Media (1996).

33. Riaz, M. "Monitoring process mean level using auxiliary information", Statistica Neerlandica, 62(4), pp. 458-481 (2008b).

34. Riaz, M. "Monitoring process variability using auxiliary information", Computational Statistics, 23(2), pp. 253-276 (2008c).
35. Riaz, M. "Control charting and survey sampling techniques in process monitoring", Journal of the Chinese Institute of Engineers, 38(3), pp. 342-354 (2015).

36. Riaz, M., Mehmood, R., Ahmad, S., and Abbasi, S.A. "On the performance of auxiliary-based control charting under normality and nonnormality with estimation effects", Quality and Reliability Engineering International, 29(8), pp. 1165-1179 (2013).

37. Ahmad, S., Riaz, M., Abbasi, S.A., and Lin, Z. "On efficient median control charting", Journal of the Chinese Institute of Engineers, 37(3), pp. 358-375 (2014a).

38. Magnus, J. "Estimation of the mean of a univariate normal distribution with known variance", The Econometrics Journal, 5(1), pp. 225-236 (2002).

39. Singh, H., Upadhyaya, L., and Chandra, P. "A general family of estimators for estimating population mean using two auxiliary variables in two-phase sampling", Statistics in Transition, 6(7), pp. 1055-1077 (2004).

40. Khoshnevisan, M., Singh, R., Chauhan, P., Sawan, N., and Smarandache, F. "A general family of estimators for estimating population mean using known value of some population parameter(s)", arXiv preprint math/0701243 (2007).

41. Ahmad, S., Riaz, M., Abbasi, S.A., and Lin, Z. "On monitoring process variability under double sampling scheme", International Journal of Production Economics, 142(2), pp. 388-400 (2013).

42. Riaz, M. "An improved control chart structure for process location parameter", Quality and Reliability Engineering International, 27(8), pp. 1033-1041 (2011).

43. Zhang, G.X. "Cause-selecting control charts - A new type of quality control charts", The QR Journal, 12(4), pp. 221-225 (1985).

44. Hawkins, D.M. "Multivariate quality control based on regression-adjusted variables", Technometrics, 33(1), pp. 61-75 (1991).

45. Hawkins, D.M. "Regression adjustment for variables in multivariate quality control", Journal of Quality Technology, 25(3), pp. 170-182 (1993).

46. Wade, M.R. and Woodall, W.H. "A review and analysis of cause-selecting control charts", Journal of Quality Technology, 25(3), pp. 161-169 (1993).

47. Shu, L., Tsung, F., and Tsui, K.-L. "Effects of estimation errors on cause-selecting charts", IIE Transactions, 37(6), pp. 559-567 (2005).

48. Abbasi, S.A. and Riaz, M. "On enhanced control charting for process monitoring", International Journal of the Physical Sciences, 8(17), pp. 759-775 (2013).

49. Abujiya, M.A.R., Riaz, M., and Lee, M.H. "Enhancing the performance of combined Shewhart-EWMA charts", Quality and Reliability Engineering International, 29(8), pp. 1093-1106 (2013). 
50. Mehmood, R., Riaz, M., and Does, R.J. "Control charts for location based on different sampling schemes", Journal of Applied Statistics, 40(3), pp. 483494 (2013).

51. Ahmad, S., Lin, Z., Abbasi, S.A., and Riaz, M. "On efficient monitoring of process dispersion using interquartile range", Open Journal of Applied Sciences, $\mathbf{2}(04)$, p. 39 (2013).

52. Ahmad, S., Riaz, M., Abbasi, S.A., and Lin, Z. "On median control charting under double sampling scheme", European Journal of Industrial Engineering, 8(4), pp. 478-512 (2014b).

53. Khoo, M.B. "A control chart based on sample median for the detection of a permanent shift in the process mean", Quality Engineering, 17(2), pp. 243-257 (2005).

54. Riaz, M. "A dispersion control chart", Communications in Statistics-Simulation and Computation ${ }^{\circledR}$, 37(6), pp. 1239-1261 (2008a).

55. Khoo, M.B. and Quah, S. "Alternatives to the multivariate control chart for process dispersion", Quality Engineering, 16(3), pp. 423-435 (2004).

56. Wen, D.-C. and Lv, J. "Multivariate process capability index based on the additivity of normal distribution", Wireless Communications, Networking and Mobile Computing, 2008. WiCOM'08. 4th International Conference on, IEEE, pp. 1-5 (2008).

57. Kuk, A.Y. and Mak, T. "Median estimation in the presence of auxiliary information", Journal of the Royal Statistical Society. Series B (Methodological), pp. 261-269 (1989).

58. Sisodia, B. and Dwivedi, V. "modified ratio estimator using coefficient of variation of auxiliary variable", Journal-Indian Society of Agricultural Statistics, (1981).

59. Yan, Z. and Tian, B. "Ratio method to the mean estimation using coefficient of skewness of auxiliary variable", In International Conference on Information Computing and Applications, Springer, Berlin, Heidelberg, pp. 103-110 (October, 2010).

60. Upadhyaya, L.N. and Singh, H.P. "Use of transformed auxiliary variable in estimating the finite popula- tion mean", Biometrical Journal, 41(5), pp. 627-636 (1999).

61. Gupta, S. and Shabbir, J. "On the use of transformed auxiliary variables in estimating population mean by using two auxiliary variables", Journal of Statistical Planning and Inference, 137(5), pp. 1606-1611 (2007).

62. Shabbir, J., Gupta, S., and Hussain, Z. "Improved estimation of finite population median under twophase sampling when using two auxiliary variables", Scientia Iranica, 22(3), pp. 1271-1277 (2015).

63. Wu, Z., Jiao, J., Yang, M., Liu, Y., and Wang, Z. "An enhanced adaptive CUSUM control chart", IIE Transactions, 41(7), pp. 642-653 (2009).

64. Schaffer, J.R. and Kim, M.-J. "Number of replications required in control chart Monte Carlo simulation studies", Communications in Statistics-Simulation and Computation $^{\circledR}$, 36(5), pp. 1075-1087 (2007).

65. Marlin, T., Process Control: Controling Processes and Control Systems for Dynamic Performance, McGrawHill, New York (2000).

66. Yoon, S. and MacGregor, J.F. "Fault diagnosis with multivariate statistical models. Part I: using steady state fault signatures", J. Process Control, 11(4), pp. 387-400 (2001).

67. Shi, X., Lv, Y., Fei, Z., and Liang, J. "A multivariable statistical process monitoring method based on multiscale analysis and principal curves", International Journal of Innovative Computing, Information and Control, 9(4), pp. 1781-1800 (2013).

\section{Appendix}

We provided some additional results of our proposed study in Tables A.1-A.4. Table A.1 describes the generated values charts $M_{1}, M_{6}$, and $M_{11}$ which we used to compare the performance of control charts in Section 5.1. Tables 1-4 represent the performance measures of control charts used in his study. The ARL values for some selective combinations are presented in Tables A.2-A.4.

Table A.1. The values of $M_{1}, M_{6}$ and, $M_{11}$.

\begin{tabular}{|c|c|c|c|c|c|c|c|c|c|c|c|c|}
\hline \multirow{3}{*}{$\begin{array}{l}\text { Sub } \\
\text { groups }\end{array}$} & \multicolumn{6}{|c|}{$\begin{array}{c}\rho_{x y}=0.90, \rho_{y z}=0.90, \rho_{z x}=0.90 \\
\lambda=0.75 \text { and } \delta=1.0\end{array}$} & \multicolumn{6}{|c|}{$\begin{array}{c}\rho_{x y}=0.90, \rho_{y z}=0.90, \rho_{z x}=0.90 \\
\lambda=0.25 \text { and } \delta=0.75\end{array}$} \\
\hline & \multicolumn{3}{|c|}{$n=5$} & \multicolumn{3}{|c|}{$n=10$} & \multicolumn{3}{|c|}{$n=5$} & \multicolumn{3}{|c|}{$n=10$} \\
\hline & $M_{1}$ & $M_{6}$ & $M_{11}$ & $M_{1}$ & $M_{6}$ & $M_{11}$ & $M_{1}$ & $M_{6}$ & $M_{11}$ & $M_{1}$ & $M_{6}$ & $M_{11}$ \\
\hline 1 & 4.529 & 4.594 & 5.246 & 4.598 & 5.115 & 5.002 & 4.998 & 4.954 & 5.052 & 4.986 & 5.041 & 4.996 \\
\hline 2 & 5.069 & 4.981 & 4.996 & 4.920 & 5.014 & 4.906 & 5.135 & 4.838 & 5.129 & 4.988 & 5.034 & 4.996 \\
\hline 3 & 4.573 & 5.073 & 5.074 & 5.352 & 4.358 & 5.003 & 5.062 & 4.936 & 5.191 & 4.951 & 5.140 & 4.987 \\
\hline 4 & 4.711 & 4.804 & 5.270 & 5.116 & 5.075 & 4.923 & 4.934 & 4.928 & 5.064 & 4.944 & 5.083 & 5.012 \\
\hline 5 & 5.140 & 5.574 & 4.883 & 4.693 & 5.252 & 4.930 & 4.865 & 4.844 & 4.859 & 4.997 & 5.014 & 5.034 \\
\hline 6 & 4.672 & 5.180 & 4.695 & 4.696 & 5.424 & 5.067 & 4.888 & 4.711 & 4.996 & 5.032 & 5.128 & 5.018 \\
\hline 7 & 4.405 & 5.569 & 4.975 & 5.127 & 5.203 & 4.925 & 4.954 & 4.865 & 4.957 & 5.115 & 5.088 & 5.010 \\
\hline
\end{tabular}


Table A.1. The values of $M_{1}, M_{6}$ and, $M_{11}$ (continued).

\begin{tabular}{|c|c|c|c|c|c|c|c|c|c|c|c|c|}
\hline \multirow{3}{*}{$\begin{array}{l}\text { Sub } \\
\text { groups }\end{array}$} & \multicolumn{6}{|c|}{$\begin{array}{c}\rho_{x y}=0.90, \rho_{y z}=0.90, \rho_{z x}=0.90 \\
\lambda=0.75 \text { and } \delta=1.0\end{array}$} & \multicolumn{6}{|c|}{$\begin{array}{c}\rho_{x y}=0.90, \rho_{y z}=0.90, \rho_{z x}=0.90 \\
\lambda=0.25 \text { and } \delta=0.75\end{array}$} \\
\hline & \multicolumn{3}{|c|}{$n=5$} & \multicolumn{3}{|c|}{$n=10$} & \multicolumn{3}{|c|}{$n=5$} & \multicolumn{3}{|c|}{$n=10$} \\
\hline & $M_{1}$ & $M_{6}$ & $M_{11}$ & $M_{1}$ & $M_{6}$ & $M_{11}$ & $M_{1}$ & $M_{6}$ & $M_{11}$ & $M_{1}$ & $M_{6}$ & $M_{11}$ \\
\hline 8 & 4.771 & 5.302 & 4.784 & 4.729 & 4.808 & 4.971 & 4.853 & 4.956 & 5.005 & 5.092 & 5.019 & 5.042 \\
\hline 9 & 4.592 & 5.393 & 4.600 & 4.553 & 4.619 & 4.870 & 5.154 & 5.102 & 4.912 & 4.959 & 4.899 & 4.963 \\
\hline 10 & 4.849 & 4.965 & 5.343 & 5.053 & 5.007 & 5.136 & 5.155 & 5.118 & 4.984 & 4.881 & 4.905 & 4.964 \\
\hline 11 & 4.674 & 4.897 & 5.222 & 5.501 & 5.009 & 5.012 & 5.066 & 4.989 & 5.013 & 4.891 & 4.914 & 4.993 \\
\hline 12 & 4.667 & 5.097 & 4.757 & 4.921 & 4.840 & 4.801 & 5.078 & 5.165 & 4.977 & 4.705 & 4.903 & 4.938 \\
\hline 13 & 5.760 & 4.755 & 4.844 & 5.210 & 4.912 & 4.834 & 5.298 & 5.172 & 4.912 & 4.800 & 4.908 & 5.098 \\
\hline 14 & 5.192 & 4.908 & 4.988 & 4.838 & 4.981 & 5.017 & 5.059 & 5.126 & 4.870 & 4.678 & 4.874 & 5.048 \\
\hline 15 & 5.321 & 5.064 & 5.488 & 4.533 & 5.442 & 5.043 & 4.845 & 4.983 & 4.864 & 4.812 & 4.864 & 5.039 \\
\hline 16 & 5.276 & 4.689 & 5.110 & 4.446 & 5.023 & 4.941 & 4.836 & 4.920 & 4.863 & 4.805 & 4.966 & 5.004 \\
\hline 17 & 4.859 & 4.513 & 4.562 & 4.702 & 5.078 & 5.097 & 4.745 & 5.013 & 4.853 & 4.747 & 5.037 & 5.007 \\
\hline 18 & 4.438 & 4.865 & 4.857 & 4.652 & 4.819 & 4.679 & 4.593 & 4.944 & 4.938 & 4.805 & 4.915 & 5.024 \\
\hline 19 & 4.785 & 4.794 & 5.366 & 4.907 & 4.996 & 5.226 & 4.551 & 4.940 & 5.044 & 4.818 & 4.811 & 5.051 \\
\hline 20 & 4.957 & 4.643 & 5.200 & 5.329 & 5.117 & 5.010 & 4.677 & 4.999 & 4.979 & 4.758 & 4.864 & 5.019 \\
\hline 21 & 5.547 & 4.789 & 4.938 & 4.855 & 4.981 & 4.953 & 4.811 & 5.093 & 5.069 & 4.745 & 4.931 & 4.965 \\
\hline 22 & 4.960 & 5.222 & 4.864 & 4.605 & 4.960 & 4.850 & 4.753 & 5.063 & 5.058 & 4.809 & 4.941 & 4.859 \\
\hline 23 & 5.164 & 5.321 & 5.220 & 4.839 & 4.485 & 5.033 & 4.965 & 4.963 & 5.072 & 4.955 & 4.955 & 4.932 \\
\hline 24 & 5.438 & 4.933 & 5.505 & 4.849 & 4.778 & 4.995 & 4.949 & 5.018 & 5.034 & 4.901 & 5.071 & 5.026 \\
\hline 25 & 5.133 & 5.071 & 5.240 & 5.021 & 5.246 & 5.105 & 5.033 & 4.926 & 5.022 & 4.941 & 5.107 & 4.990 \\
\hline 26 & 5.154 & 5.230 & 5.155 & 4.709 & 4.605 & 4.962 & 5.020 & 4.747 & 5.029 & 4.935 & 5.070 & 5.045 \\
\hline 27 & 5.047 & 5.073 & 4.786 & 5.331 & 5.376 & 4.836 & 4.926 & 4.732 & 4.943 & 4.887 & 5.036 & 5.026 \\
\hline 28 & 4.930 & 4.804 & 4.956 & 5.558 & 4.938 & 5.077 & 4.874 & 5.024 & 4.966 & 5.056 & 5.176 & 5.125 \\
\hline 29 & 4.436 & 5.142 & 4.547 & 5.401 & 5.269 & 5.262 & 4.804 & 4.920 & 4.979 & 5.063 & 5.027 & 5.083 \\
\hline 30 & 5.023 & 4.945 & 5.109 & 5.283 & 4.865 & 5.312 & 4.666 & 4.870 & 4.960 & 4.967 & 4.998 & 5.036 \\
\hline 31 & 5.541 & 5.482 & 5.912 & 5.095 & 5.735 & 6.110 & 4.857 & 5.028 & 5.105 & 5.137 & 5.181 & 5.267 \\
\hline 32 & 5.635 & 6.132 & 5.734 & 5.391 & 6.046 & 5.985 & 4.895 & 5.112 & 5.234 & 5.175 & 5.231 & 5.337 \\
\hline 33 & 5.632 & 5.884 & 6.076 & 6.602 & 6.073 & 5.935 & 4.877 & 5.356 & 5.418 & 5.325 & 5.327 & 5.520 \\
\hline 34 & 5.547 & 6.308 & 5.484 & 6.274 & 6.036 & 5.863 & 4.944 & 5.384 & 5.532 & 5.345 & 5.614 & 5.573 \\
\hline 35 & 5.718 & 6.468 & 5.798 & 5.939 & 5.936 & 5.757 & 5.132 & 5.643 & 5.603 & 5.544 & 5.777 & 5.651 \\
\hline 36 & 6.062 & 6.098 & 5.777 & 6.242 & 5.793 & 5.861 & 5.248 & 5.510 & 5.696 & 5.506 & 5.725 & 5.657 \\
\hline 37 & 5.715 & 6.580 & 6.156 & 5.663 & 6.253 & 6.128 & 5.335 & 5.664 & 5.824 & 5.390 & 5.702 & 5.614 \\
\hline 38 & 5.784 & 6.046 & 6.419 & 5.579 & 6.083 & 5.734 & 5.301 & 5.792 & 5.734 & 5.496 & 5.724 & 5.638 \\
\hline 39 & 5.451 & 5.666 & 6.117 & 6.044 & 5.681 & 6.046 & 5.529 & 5.836 & 5.761 & 5.475 & 5.710 & 5.668 \\
\hline 40 & 5.959 & 6.228 & 5.794 & 6.386 & 5.694 & 5.843 & 5.558 & 5.940 & 5.643 & 5.589 & 5.633 & 5.642 \\
\hline 41 & 6.719 & 6.068 & 6.110 & 5.882 & 5.971 & 5.635 & 5.727 & 6.075 & 5.681 & 5.678 & 5.541 & 5.718 \\
\hline 42 & 5.795 & 5.829 & 5.913 & 5.704 & 5.742 & 5.797 & 5.697 & 5.951 & 5.732 & 5.826 & 5.589 & 5.734 \\
\hline 43 & 5.917 & 5.860 & 5.942 & 5.531 & 5.636 & 6.210 & 5.684 & 5.798 & 5.693 & 5.916 & 5.592 & 5.647 \\
\hline 44 & 5.573 & 5.849 & 5.879 & 5.916 & 5.880 & 6.271 & 5.768 & 5.710 & 5.745 & 5.813 & 5.647 & 5.641 \\
\hline 45 & 5.736 & 6.084 & 5.626 & 5.905 & 5.704 & 6.334 & 5.896 & 5.821 & 5.763 & 5.908 & 5.703 & 5.717 \\
\hline 46 & 6.456 & 6.471 & 5.993 & 6.244 & 6.078 & 6.002 & 5.848 & 5.692 & 5.768 & 5.716 & 5.793 & 5.745 \\
\hline 47 & 6.387 & 6.674 & 5.794 & 5.493 & 5.916 & 5.949 & 5.783 & 5.773 & 5.744 & 5.776 & 5.761 & 5.773 \\
\hline 48 & 6.019 & 6.280 & 6.005 & 5.667 & 5.876 & 6.053 & 6.026 & 5.778 & 5.809 & 5.569 & 5.867 & 5.784 \\
\hline 49 & 5.762 & 6.456 & 6.382 & 6.218 & 6.202 & 6.154 & 6.166 & 5.864 & 5.744 & 5.615 & 5.733 & 5.762 \\
\hline 50 & 5.908 & 6.235 & 6.173 & 5.928 & 5.864 & 6.218 & 6.161 & 5.744 & 5.658 & 5.675 & 5.678 & 5.740 \\
\hline
\end{tabular}


Table A.2. ARL values for EWMA median charts with $n=5, \rho_{x y}=0.90, \rho_{y z}=0.75$, and $\rho_{z x}=0.50$.

\begin{tabular}{|c|c|c|c|c|c|c|c|c|c|c|c|c|}
\hline$\delta$ & $\lambda$ & $M_{1}$ & $M_{2}$ & $M_{3}$ & $M_{4}$ & $M_{5}$ & $M_{6}$ & $M_{7}$ & $M_{8}$ & $M_{9}$ & $M_{10}$ & $M_{11}$ \\
\hline \multirow{4}{*}{0.00} & 1.00 & 200.24 & 200.00 & 200.15 & 200.41 & 200.13 & 200.67 & 199.98 & 200.18 & 200.20 & 200.11 & 200.18 \\
\hline & 0.75 & 200.11 & 199.71 & 200.00 & 200.00 & 200.00 & 200.64 & 199.83 & 200.18 & 200.70 & 199.96 & 199.62 \\
\hline & 0.50 & 200.14 & 199.98 & 200.00 & 200.12 & 200.10 & 199.87 & 199.73 & 200.18 & 199.96 & 200.18 & 200.02 \\
\hline & 0.25 & 200.28 & 199.53 & 200.44 & 200.28 & 200.38 & 199.97 & 200.02 & 199.97 & 200.37 & 200.00 & 200.26 \\
\hline \multirow{4}{*}{0.10} & 1.00 & 176.06 & 119.24 & 121.81 & 175.09 & 120.37 & 197.97 & 171.41 & 153.16 & 155.70 & 153.23 & 159.61 \\
\hline & 0.75 & 162.62 & 105.02 & 107.93 & 151.19 & 106.81 & 176.86 & 158.79 & 129.82 & 131.08 & 129.99 & 134.40 \\
\hline & 0.50 & 144.86 & 87.76 & 88.51 & 115.56 & 87.70 & 145.91 & 133.67 & 94.24 & 94.68 & 94.20 & 96.15 \\
\hline & 0.25 & 116.48 & 65.13 & 64.68 & 72.33 & 64.87 & 96.86 & 104.63 & 55.42 & 55.40 & 55.48 & 55.53 \\
\hline \multirow{4}{*}{0.15} & 1.00 & 151.82 & 91.80 & 94.18 & 138.75 & 93.12 & 169.80 & 147.23 & 112.92 & 114.55 & 112.83 & 116.85 \\
\hline & 0.75 & 132.01 & 72.37 & 73.67 & 104.97 & 73.05 & 134.96 & 125.40 & 81.79 & 82.68 & 81.58 & 83.92 \\
\hline & 0.50 & 105.42 & 53.95 & 53.34 & 66.90 & 53.64 & 96.08 & 93.44 & 51.78 & 52.10 & 51.66 & 53.13 \\
\hline & 0.25 & 71.11 & 34.63 & 34.02 & 34.97 & 34.42 & 54.80 & 62.38 & 24.61 & 24.30 & 24.61 & 24.01 \\
\hline \multirow{4}{*}{0.20} & 1.00 & 124.18 & 67.51 & 66.83 & 97.71 & 67.11 & 134.10 & 119.21 & 78.48 & 80.09 & 78.46 & 82.32 \\
\hline & 0.75 & 99.36 & 52.23 & 52.41 & 64.85 & 52.16 & 93.37 & 93.78 & 52.48 & 52.59 & 52.50 & 53.32 \\
\hline & 0.50 & 74.82 & 35.34 & 34.55 & 40.06 & 35.01 & 60.74 & 64.87 & 28.23 & 28.17 & 28.18 & 28.22 \\
\hline & 0.25 & 46.81 & 19.57 & 18.90 & 18.22 & 19.19 & 31.85 & 38.50 & 11.97 & 11.68 & 12.00 & 11.37 \\
\hline \multirow{4}{*}{0.25} & 1.00 & 100.62 & 49.57 & 49.61 & 69.48 & 49.85 & 101.32 & 92.70 & 54.29 & 55.65 & 54.23 & 57.16 \\
\hline & 0.75 & 76.58 & 36.43 & 35.55 & 42.67 & 36.29 & 67.00 & 66.66 & 31.96 & 32.24 & 31.93 & 33.56 \\
\hline & 0.50 & 51.56 & 23.43 & 22.60 & 24.49 & 22.93 & 39.75 & 44.94 & 16.13 & 16.07 & 16.15 & 16.34 \\
\hline & 0.25 & 30.91 & 11.74 & 11.10 & 10.14 & 11.44 & 19.26 & 24.82 & 5.91 & 5.62 & 5.94 & 5.31 \\
\hline \multirow{4}{*}{0.50} & 1.00 & 34.70 & 13.41 & 12.89 & 14.09 & 13.17 & 26.38 & 27.21 & 9.44 & 9.21 & 9.41 & 9.47 \\
\hline & 0.75 & 20.39 & 8.01 & 7.51 & 6.96 & 7.87 & 13.52 & 16.05 & 4.71 & 4.67 & 4.70 & 4.83 \\
\hline & 0.50 & 11.88 & 4.29 & 3.88 & 3.51 & 4.13 & 7.02 & 9.01 & 2.12 & 2.07 & 2.12 & 2.02 \\
\hline & 0.25 & 4.58 & 1.16 & 1.13 & 1.06 & 1.15 & 1.94 & 2.92 & 1.00 & 1.00 & 1.00 & 1.00 \\
\hline \multirow{4}{*}{0.75} & 1.00 & 13.18 & 4.92 & 4.55 & 4.22 & 4.79 & 8.25 & 10.04 & 2.67 & 2.67 & 2.66 & 2.67 \\
\hline & 0.75 & 7.26 & 2.76 & 2.57 & 2.24 & 2.70 & 4.27 & 5.44 & 1.51 & 1.50 & 1.52 & 1.49 \\
\hline & 0.50 & 3.89 & 1.37 & 1.31 & 1.18 & 1.35 & 2.00 & 2.69 & 1.03 & 1.03 & 1.03 & 1.03 \\
\hline & 0.25 & 1.14 & 1.00 & 1.00 & 1.00 & 1.00 & 1.00 & 1.02 & 1.00 & 1.00 & 1.00 & 1.00 \\
\hline \multirow{4}{*}{1.0} & 1.00 & 5.86 & 2.37 & 2.14 & 1.89 & 2.26 & 3.45 & 4.48 & 1.37 & 1.38 & 1.36 & 1.36 \\
\hline & 0.75 & 3.44 & 1.40 & 1.37 & 1.22 & 1.40 & 1.90 & 2.45 & 1.05 & 1.05 & 1.05 & 1.06 \\
\hline & 0.50 & 1.66 & 1.02 & 1.01 & 1.00 & 1.01 & 1.13 & 1.29 & 1.00 & 1.00 & 1.00 & 1.00 \\
\hline & 0.25 & 1.00 & 1.00 & 1.00 & 1.00 & 1.00 & 1.00 & 1.00 & 1.00 & 1.00 & 1.00 & 1.00 \\
\hline \multirow{4}{*}{1.25} & 1.00 & 3.20 & 1.46 & 1.42 & 1.23 & 1.43 & 1.95 & 2.40 & 1.08 & 1.08 & 1.07 & 1.08 \\
\hline & 0.75 & 1.83 & 1.07 & 1.06 & 1.02 & 1.07 & 1.24 & 1.47 & 1.00 & 1.00 & 1.00 & 1.00 \\
\hline & 0.50 & 1.13 & 1.00 & 1.00 & 1.00 & 1.00 & 1.01 & 1.02 & 1.00 & 1.00 & 1.00 & 1.00 \\
\hline & 0.25 & 1.00 & 1.00 & 1.00 & 1.00 & 1.00 & 1.00 & 1.00 & 1.00 & 1.00 & 1.00 & 1.00 \\
\hline \multirow{4}{*}{1.50} & 1.00 & 2.05 & 1.16 & 1.13 & 1.06 & 1.16 & 1.35 & 1.61 & 1.01 & 1.01 & 1.01 & 1.01 \\
\hline & 0.75 & 1.32 & 1.01 & 1.00 & 1.00 & 1.01 & 1.04 & 1.13 & 1.00 & 1.00 & 1.00 & 1.00 \\
\hline & 0.50 & 1.01 & 1.00 & 1.00 & 1.00 & 1.00 & 1.00 & 1.00 & 1.00 & 1.00 & 1.00 & 1.00 \\
\hline & 0.25 & 1.00 & 1.00 & 1.00 & 1.00 & 1.00 & 1.00 & 1.00 & 1.00 & 1.00 & 1.00 & 1.00 \\
\hline \multirow{4}{*}{2.00} & 1.00 & 1.22 & 1.01 & 1.01 & 1.00 & 1.01 & 1.03 & 1.10 & 1.00 & 1.00 & 1.00 & 1.00 \\
\hline & 0.75 & 1.02 & 1.00 & 1.00 & 1.00 & 1.00 & 1.00 & 1.00 & 1.00 & 1.00 & 1.00 & 1.00 \\
\hline & 0.50 & 1.00 & 1.00 & 1.00 & 1.00 & 1.00 & 1.00 & 1.00 & 1.00 & 1.00 & 1.00 & 1.00 \\
\hline & 0.25 & 1.00 & 1.00 & 1.00 & 1.00 & 1.00 & 1.00 & 1.00 & 1.00 & 1.00 & 1.00 & 1.00 \\
\hline
\end{tabular}


Table A.3. ARL values for EWMA median charts with $n=10, \rho_{x y}=0.75, \rho_{y z}=0.10$, and $\rho_{z x}=0.10$.

\begin{tabular}{|c|c|c|c|c|c|c|c|c|c|c|c|c|}
\hline$\delta$ & $\lambda$ & $M_{1}$ & $M_{2}$ & $M_{3}$ & $M_{4}$ & $M_{5}$ & $M_{6}$ & $M_{7}$ & $M_{8}$ & $M_{9}$ & $M_{10}$ & $M_{11}$ \\
\hline \multirow{4}{*}{0.00} & 1.00 & 200.26 & 200.21 & 199.62 & 199.99 & 199.48 & 199.88 & 200.22 & 200.06 & 199.93 & 199.93 & 200.42 \\
\hline & 0.75 & 200.09 & 200.12 & 200.14 & 199.91 & 199.97 & 200.15 & 200.26 & 200.18 & 200.37 & 199.97 & 200.06 \\
\hline & 0.50 & 200.40 & 200.12 & 200.46 & 200.33 & 200.14 & 199.73 & 199.97 & 200.08 & 200.18 & 200.06 & 200.07 \\
\hline & 0.25 & 200.22 & 199.84 & 199.84 & 199.96 & 199.88 & 200.16 & 199.78 & 199.72 & 199.97 & 199.76 & 200.07 \\
\hline \multirow{4}{*}{0.10} & 1.00 & 146.04 & 110.75 & 110.44 & 132.07 & 110.18 & 153.41 & 136.28 & 136.99 & 137.09 & 137.07 & 137.66 \\
\hline & 0.75 & 129.64 & 96.56 & 95.87 & 107.64 & 96.38 & 129.59 & 113.01 & 113.77 & 113.50 & 113.34 & 113.29 \\
\hline & 0.50 & 105.89 & 80.55 & 80.62 & 81.22 & 80.56 & 98.75 & 83.56 & 83.37 & 83.66 & 83.29 & 84.31 \\
\hline & 0.25 & 75.67 & 59.38 & 58.84 & 52.94 & 59.35 & 63.29 & 52.96 & 53.70 & 53.50 & 53.63 & 53.31 \\
\hline \multirow{4}{*}{0.15} & 1.00 & 113.29 & 78.98 & 79.55 & 92.74 & 79.28 & 111.77 & 96.94 & 97.00 & 96.95 & 96.98 & 96.99 \\
\hline & 0.75 & 88.63 & 64.44 & 63.27 & 67.60 & 63.71 & 81.67 & 70.50 & 70.40 & 70.27 & 69.98 & 70.33 \\
\hline & 0.50 & 63.61 & 48.97 & 48.25 & 45.87 & 48.78 & 54.95 & 46.79 & 46.48 & 46.41 & 46.41 & 46.50 \\
\hline & 0.25 & 38.53 & 31.34 & 30.38 & 25.48 & 31.05 & 31.13 & 24.78 & 24.95 & 24.83 & 24.97 & 24.77 \\
\hline \multirow{4}{*}{0.20} & 1.00 & 83.96 & 57.72 & 56.68 & 63.99 & 56.83 & 78.97 & 65.03 & 65.20 & 65.13 & 65.16 & 65.53 \\
\hline & 0.75 & 60.42 & 42.51 & 41.59 & 41.60 & 42.03 & 52.22 & 42.04 & 41.89 & 41.94 & 41.94 & 42.06 \\
\hline & 0.50 & 40.25 & 29.82 & 28.94 & 25.82 & 29.61 & 31.77 & 26.13 & 25.98 & 26.12 & 25.99 & 26.05 \\
\hline & 0.25 & 22.13 & 17.24 & 16.41 & 12.84 & 16.95 & 16.05 & 12.76 & 12.78 & 12.67 & 12.78 & 12.58 \\
\hline \multirow{4}{*}{0.25} & 1.00 & 58.85 & 43.45 & 42.79 & 41.91 & 43.38 & 53.34 & 44.15 & 43.82 & 43.81 & 43.78 & 44.24 \\
\hline & 0.75 & 40.55 & 29.84 & 28.63 & 25.70 & 29.41 & 33.11 & 27.11 & 27.11 & 27.16 & 27.06 & 27.35 \\
\hline & 0.50 & 24.90 & 18.99 & 18.39 & 15.47 & 18.75 & 19.58 & 15.57 & 15.55 & 15.55 & 15.56 & 15.49 \\
\hline & 0.25 & 12.88 & 9.87 & 9.31 & 6.69 & 9.71 & 8.43 & 6.46 & 6.45 & 6.34 & 6.46 & 6.25 \\
\hline \multirow{4}{*}{0.50} & 1.00 & 14.00 & 10.39 & 9.81 & 8.12 & 10.16 & 10.29 & 8.19 & 8.11 & 8.13 & 8.12 & 8.22 \\
\hline & 0.75 & 7.90 & 6.32 & 5.89 & 4.44 & 6.24 & 5.71 & 4.41 & 4.38 & 4.38 & 4.40 & 4.34 \\
\hline & 0.50 & 4.32 & 3.36 & 3.23 & 2.19 & 3.32 & 2.78 & 2.23 & 2.24 & 2.20 & 2.25 & 2.20 \\
\hline & 0.25 & 1.22 & 1.06 & 1.05 & 1.01 & 1.06 & 1.03 & 1.01 & 1.01 & 1.01 & 1.01 & 1.01 \\
\hline \multirow{4}{*}{0.75} & 1.00 & 4.80 & 3.73 & 3.59 & 2.63 & 3.70 & 3.30 & 2.65 & 2.64 & 2.65 & 2.64 & 2.60 \\
\hline & 0.75 & 2.70 & 2.20 & 2.13 & 1.61 & 2.15 & 1.89 & 1.55 & 1.55 & 1.55 & 1.56 & 1.54 \\
\hline & 0.50 & 1.39 & 1.22 & 1.18 & 1.05 & 1.19 & 1.10 & 1.04 & 1.04 & 1.04 & 1.04 & 1.04 \\
\hline & 0.25 & 1.00 & 1.00 & 1.00 & 1.00 & 1.00 & 1.00 & 1.00 & 1.00 & 1.00 & 1.00 & 1.00 \\
\hline \multirow{4}{*}{1.0} & 1.00 & 2.23 & 1.93 & 1.81 & 1.43 & 1.87 & 1.63 & 1.43 & 1.42 & 1.42 & 1.42 & 1.42 \\
\hline & 0.75 & 1.39 & 1.24 & 1.20 & 1.07 & 1.22 & 1.13 & 1.06 & 1.06 & 1.06 & 1.06 & 1.06 \\
\hline & 0.50 & 1.02 & 1.01 & 1.00 & 1.00 & 1.00 & 1.00 & 1.00 & 1.00 & 1.00 & 1.00 & 1.00 \\
\hline & 0.25 & 1.00 & 1.00 & 1.00 & 1.00 & 1.00 & 1.00 & 1.00 & 1.00 & 1.00 & 1.00 & 1.00 \\
\hline \multirow{4}{*}{1.25} & 1.00 & 1.40 & 1.29 & 1.25 & 1.10 & 1.29 & 1.16 & 1.09 & 1.08 & 1.08 & 1.08 & 1.09 \\
\hline & 0.75 & 1.07 & 1.04 & 1.03 & 1.00 & 1.03 & 1.01 & 1.00 & 1.00 & 1.00 & 1.00 & 1.00 \\
\hline & 0.50 & 1.00 & 1.00 & 1.00 & 1.00 & 1.00 & 1.00 & 1.00 & 1.00 & 1.00 & 1.00 & 1.00 \\
\hline & 0.25 & 1.00 & 1.00 & 1.00 & 1.00 & 1.00 & 1.00 & 1.00 & 1.00 & 1.00 & 1.00 & 1.00 \\
\hline \multirow{4}{*}{1.50} & 1.00 & 1.13 & 1.09 & 1.07 & 1.02 & 1.08 & 1.03 & 1.01 & 1.01 & 1.01 & 1.01 & 1.01 \\
\hline & 0.75 & 1.01 & 1.00 & 1.00 & 1.00 & 1.00 & 1.00 & 1.00 & 1.00 & 1.00 & 1.00 & 1.00 \\
\hline & 0.50 & 1.00 & 1.00 & 1.00 & 1.00 & 1.00 & 1.00 & 1.00 & 1.00 & 1.00 & 1.00 & 1.00 \\
\hline & 0.25 & 1.00 & 1.00 & 1.00 & 1.00 & 1.00 & 1.00 & 1.00 & 1.00 & 1.00 & 1.00 & 1.00 \\
\hline \multirow{4}{*}{2.00} & 1.00 & 1.00 & 1.00 & 1.00 & 1.00 & 1.00 & 1.00 & 1.00 & 1.00 & 1.00 & 1.00 & 1.00 \\
\hline & 0.75 & 1.00 & 1.00 & 1.00 & 1.00 & 1.00 & 1.00 & 1.00 & 1.00 & 1.00 & 1.00 & 1.00 \\
\hline & 0.50 & 1.00 & 1.00 & 1.00 & 1.00 & 1.00 & 1.00 & 1.00 & 1.00 & 1.00 & 1.00 & 1.00 \\
\hline & 0.25 & 1.00 & 1.00 & 1.00 & 1.00 & 1.00 & 1.00 & 1.00 & 1.00 & 1.00 & 1.00 & 1.00 \\
\hline
\end{tabular}


Table A.4. ARL values for EWMA median charts with $n=15, \rho_{x y}=0.50, \rho_{y z}=0.50$, and $\rho_{z x}=0.10$.

\begin{tabular}{|c|c|c|c|c|c|c|c|c|c|c|c|c|}
\hline$\delta$ & $\lambda$ & $M_{1}$ & $M_{2}$ & $M_{3}$ & $M_{4}$ & $M_{5}$ & $M_{6}$ & $M_{7}$ & $M_{8}$ & $M_{9}$ & $M_{10}$ & $M_{11}$ \\
\hline \multirow{4}{*}{0.00} & 1.00 & 199.93 & 199.71 & 200.43 & 199.86 & 199.85 & 200.02 & 200.21 & 199.95 & 200.29 & 200.11 & 199.95 \\
\hline & 0.75 & 200.33 & 200.00 & 200.00 & 199.94 & 200.43 & 200.44 & 200.10 & 200.32 & 200.39 & 200.39 & 200.29 \\
\hline & 0.50 & 200.99 & 200.19 & 200.00 & 200.34 & 200.43 & 199.84 & 200.10 & 199.93 & 200.10 & 199.93 & 200.21 \\
\hline & 0.25 & 200.28 & 200.17 & 200.26 & 200.38 & 200.17 & 200.19 & 200.18 & 199.94 & 199.73 & 199.90 & 199.56 \\
\hline \multirow{4}{*}{0.10} & 1.00 & 146.45 & 114.83 & 116.31 & 125.12 & 115.11 & 140.46 & 136.42 & 138.83 & 139.44 & 138.80 & 138.99 \\
\hline & 0.75 & 122.51 & 102.10 & 102.19 & 106.34 & 101.81 & 114.00 & 114.38 & 114.60 & 114.00 & 114.52 & 113.87 \\
\hline & 0.50 & 93.38 & 88.45 & 87.02 & 85.96 & 87.97 & 85.07 & 84.85 & 85.20 & 85.10 & 85.22 & 85.47 \\
\hline & 0.25 & 59.51 & 69.17 & 68.50 & 57.52 & 68.76 & 56.90 & 55.58 & 54.93 & 54.82 & 54.89 & 54.84 \\
\hline \multirow{4}{*}{0.15} & 1.00 & 100.52 & 86.25 & 86.30 & 88.08 & 86.12 & 96.98 & 93.28 & 93.26 & 93.83 & 93.36 & 93.31 \\
\hline & 0.75 & 74.49 & 70.41 & 69.75 & 66.86 & 70.31 & 69.89 & 69.53 & 69.20 & 68.98 & 69.21 & 68.72 \\
\hline & 0.50 & 51.96 & 54.97 & 53.68 & 48.21 & 54.89 & 46.27 & 46.06 & 46.08 & 45.89 & 46.06 & 45.82 \\
\hline & 0.25 & 29.88 & 37.53 & 36.47 & 27.90 & 37.07 & 26.16 & 26.80 & 26.72 & 26.70 & 26.74 & 26.60 \\
\hline \multirow{4}{*}{0.20} & 1.00 & 67.82 & 64.11 & 63.52 & 61.78 & 63.79 & 64.77 & 63.32 & 63.84 & 64.07 & 63.88 & 63.58 \\
\hline & 0.75 & 47.22 & 49.40 & 48.59 & 43.17 & 49.19 & 44.04 & 42.69 & 42.17 & 42.27 & 42.18 & 42.27 \\
\hline & 0.50 & 30.48 & 35.85 & 34.75 & 27.51 & 35.71 & 25.60 & 26.53 & 26.42 & 26.32 & 26.41 & 26.45 \\
\hline & 0.25 & 15.29 & 21.71 & 20.87 & 15.00 & 21.36 & 13.54 & 13.13 & 13.31 & 13.30 & 13.31 & 13.19 \\
\hline \multirow{4}{*}{0.25} & 1.00 & 47.74 & 47.23 & 46.82 & 42.21 & 46.88 & 43.27 & 43.98 & 43.42 & 43.42 & 43.47 & 43.39 \\
\hline & 0.75 & 30.40 & 34.51 & 33.48 & 27.59 & 34.26 & 27.18 & 26.88 & 26.66 & 26.54 & 26.66 & 26.59 \\
\hline & 0.50 & 18.30 & 23.36 & 22.24 & 17.59 & 22.97 & 15.62 & 15.50 & 15.47 & 15.52 & 15.47 & 15.45 \\
\hline & 0.25 & 8.31 & 13.00 & 12.36 & 7.96 & 12.76 & 6.81 & 6.78 & 6.68 & 6.67 & 6.69 & 6.61 \\
\hline \multirow{4}{*}{0.50} & 1.00 & 9.47 & 12.98 & 12.55 & 9.03 & 12.80 & 8.12 & 8.29 & 8.18 & 8.30 & 8.20 & 8.15 \\
\hline & 0.75 & 5.30 & 7.99 & 7.50 & 5.12 & 7.82 & 4.50 & 4.72 & 4.68 & 4.63 & 4.68 & 4.58 \\
\hline & 0.50 & 2.77 & 4.55 & 4.29 & 2.69 & 4.47 & 2.30 & 2.29 & 2.26 & 2.25 & 2.26 & 2.25 \\
\hline & 0.25 & 1.03 & 1.30 & 1.21 & 1.02 & 1.26 & 1.01 & 1.01 & 1.01 & 1.01 & 1.01 & 1.00 \\
\hline \multirow{4}{*}{0.75} & 1.00 & 3.08 & 4.86 & 4.58 & 3.05 & 4.76 & 2.69 & 2.72 & 2.65 & 2.66 & 2.66 & 2.65 \\
\hline & 0.75 & 1.84 & 2.87 & 2.75 & 1.86 & 2.84 & 1.61 & 1.62 & 1.62 & 1.62 & 1.62 & 1.62 \\
\hline & 0.50 & 1.11 & 1.48 & 1.41 & 1.08 & 1.45 & 1.05 & 1.04 & 1.04 & 1.04 & 1.04 & 1.04 \\
\hline & 0.25 & 1.00 & 1.00 & 1.00 & 1.00 & 1.00 & 1.00 & 1.00 & 1.00 & 1.00 & 1.00 & 1.00 \\
\hline \multirow{4}{*}{1.0} & 1.00 & 1.60 & 2.39 & 2.28 & 1.59 & 2.33 & 1.45 & 1.44 & 1.43 & 1.44 & 1.42 & 1.43 \\
\hline & 0.75 & 1.12 & 1.48 & 1.44 & 1.13 & 1.45 & 1.08 & 1.07 & 1.07 & 1.07 & 1.07 & 1.08 \\
\hline & 0.50 & 1.00 & 1.02 & 1.02 & 1.00 & 1.03 & 1.00 & 1.00 & 1.00 & 1.00 & 1.00 & 1.00 \\
\hline & 0.25 & 1.00 & 1.00 & 1.00 & 1.00 & 1.00 & 1.00 & 1.00 & 1.00 & 1.00 & 1.00 & 1.00 \\
\hline \multirow{4}{*}{1.25} & 1.00 & 1.15 & 1.53 & 1.46 & 1.16 & 1.50 & 1.10 & 1.10 & 1.10 & 1.10 & 1.10 & 1.10 \\
\hline & 0.75 & 1.01 & 1.10 & 1.08 & 1.01 & 1.09 & 1.00 & 1.00 & 1.00 & 1.00 & 1.00 & 1.00 \\
\hline & 0.50 & 1.00 & 1.00 & 1.00 & 1.00 & 1.00 & 1.00 & 1.00 & 1.00 & 1.00 & 1.00 & 1.00 \\
\hline & 0.25 & 1.00 & 1.00 & 1.00 & 1.00 & 1.00 & 1.00 & 1.00 & 1.00 & 1.00 & 1.00 & 1.00 \\
\hline \multirow{4}{*}{1.50} & 1.00 & 1.03 & 1.18 & 1.15 & 1.03 & 1.17 & 1.02 & 1.01 & 1.02 & 1.02 & 1.02 & 1.02 \\
\hline & 0.75 & 1.00 & 1.01 & 1.01 & 1.00 & 1.01 & 1.00 & 1.00 & 1.00 & 1.00 & 1.00 & 1.00 \\
\hline & 0.50 & 1.00 & 1.00 & 1.00 & 1.00 & 1.00 & 1.00 & 1.00 & 1.00 & 1.00 & 1.00 & 1.00 \\
\hline & 0.25 & 1.00 & 1.00 & 1.00 & 1.00 & 1.00 & 1.00 & 1.00 & 1.00 & 1.00 & 1.00 & 1.00 \\
\hline \multirow{4}{*}{2.00} & 1.00 & 1.00 & 1.01 & 1.01 & 1.00 & 1.01 & 1.00 & 1.00 & 1.00 & 1.00 & 1.00 & 1.00 \\
\hline & 0.75 & 1.00 & 1.00 & 1.00 & 1.00 & 1.00 & 1.00 & 1.00 & 1.00 & 1.00 & 1.00 & 1.00 \\
\hline & 0.50 & 1.00 & 1.00 & 1.00 & 1.00 & 1.00 & 1.00 & 1.00 & 1.00 & 1.00 & 1.00 & 1.00 \\
\hline & 0.25 & 1.00 & 1.00 & 1.00 & 1.00 & 1.00 & 1.00 & 1.00 & 1.00 & 1.00 & 1.00 & 1.00 \\
\hline
\end{tabular}




\section{Biographies}

Shahid Hussain obtained his MSc degree in Statistics in 2006, and MPhil in Statistics in 2008 from the Department of Statistics, Quaid-i-Azam University, Islamabad, Pakistan. Currently, he is working on his $\mathrm{PhD}$ focusing on different charting techniques using median estimators at the Dalian University of Technology, Dalian, China. He is an Assistant Professor in the COMSATS Institute of Information Technology, Attock Campus, Pakistan. His current research interests include Statistical Process Control (SPC) and application of sampling techniques.

Lixin Song obtained his PhD degree in Statistics from the Academy of Mathematics and Systems Science, CAS, Beijing, China, in 1993. He holds the position of professorship in the School of Mathematical Sciences, Dalian University of Technology, Dalian, China. His current research interests include mathematical statistics and financial mathematics.

Shabbir Ahmad obtained his MSc degree in Statistics from the PMAS-Arid Agriculture University Rawalpindi, Pakistan, in 2002, and his MPhil in Statistics from Quaid-i-Azam University, Islamabad, Pakistan, in 2005. He served as a statistical officer in National Accounts Wing, Pakistan Bureau of Statistics (PBS), during 2006-2007. He obtained his PhD degree in Statistics from the Department of Mathematics, Institute of Statistics, Zhejiang University, Hangzhou, China, in 2013. He is serving as an Assistant Professor in the Department of Mathematics, COMSATS Institute of Information Technology, Wah Cantt, Pakistan. His current research interests include Statistical Process Control (SPC) and application of sampling techniques.

Muhammad Riaz earned the MSc in Statistics from the Department of Mathematics and Statistics at Quaid-i-Azam University, Islamabad, Pakistan, in 2001 and the PhD degree in Statistics from the Institute of Business and Industrial Statistics, University of Amsterdam, The Netherlands, in 2008. He served as a statistical officer in MINFAL Pakistan during 20022003; as a staff demographer in PIDE Pakistan during 2003-2004; as a lecturer in the Department of Statistics, Quaid-i-Azam University, Islamabad, Pakistan, during 2004-2007, and as an Assistant Professor in the Department of Statistics, Quaid-i-Azam University, Islamabad, Pakistan, during 2007-2010. He is serving as a Professor in the Department of Mathematics and Statistics, King Fahad University of Petroleum and Minerals, Dhahran 31261, Saudi Arabia.

His current research interests include statistical process control, non-parametric techniques, experimental designs, and computational statistics (focusing on code development). 